\title{
Another infinite tri-Sasaki family and marginal deformations
}

\author{
Osvaldo Pablo Santillan \\ School of Mathematics, Hamilton Building, Trinity College Dublin \\ osantil@math.tcd.ie, firenzecita@hotmail.com
}

\begin{abstract}
Several Einstein-Sasaki seven-metrics appearing in the physical literature are fibred over four-dimensional Kahler-Einstein metrics. Instead we consider here the natural Kahler-Einstein metrics defined over the twistor space $Z$ of any quaternion Kahler four-space, together with the corresponding Einstein-Sasaki metrics. We work out an explicit expression for these metrics and we prove that they are indeed tri-Sasaki. Moreover, we present a squashed version of them which is of weak $G_{2}$ holonomy. We focus in examples with three commuting Killing vectors and we extend them to supergravity backgrounds with $T^{3}$ isometry, some of them with $A d S_{4} \times X_{7}$ near horizon limit and some others without this property. We would like to emphasize that there is an underlying linear structure describing these spaces. We also consider the effect of the $S L(2, R)$ solution-generating technique presented by Maldacena and Lunin to these backgrounds and we find some rotating membrane configurations reproducing the $\mathrm{E}-\mathrm{S}$ logarithmic behaviour.
\end{abstract}

\section{Introduction}

A duality between quantum field theories and strings was proposed by t' Hooft in [1], where it was noticed that any Feynman diagram of an $U(N)$

e-print archive: http://lanl.arXiv.org/abs/hep-th/0701112v5 
gauge theory with matter fields can be drawn over a two-dimensional surface. Few of these diagrams corresponds to a plane or to a sphere, higher diagrams are drawn over a surface with non-trivial genus, such as the torus. Any closed line contributes with a factor $N$ to the amplitude. The partition function results in an expansion in terms of the form $c_{g}(\lambda) N^{2-2 h} h$ being the genus (or the number of holes) of the two-dimensional surface and $c_{g}(\lambda)$ certain function of the t' Hooft coupling $\lambda=g^{2} N$. In the large $N$ limit and keeping fixed $\lambda$ the powers $N^{2-2 h}$ goes all to zero except for diagrams with $h=0,1$. For this reason this limit is called the planar limit. If $\lambda \ll 1$ then $g \ll 1$, this corresponds to the perturbative regime. On the other hand for $\lambda \gg 1$ the amplitudes have been found to be a sum of terms of the form $A_{g}(\lambda) g^{h-1}$. In practice $N=3$ can be considered as a large number of colours and this expansion corresponds to non-perturbative phenomena [1,2]. If in addition the variable $\lambda$ became large then more diagrams contribute and become dense in the sphere. It was suggested [1] that this diagrammatic expansion is describing a discrete version of string theory in which $A_{g}$ is interpreted as a closed string amplitude. This string theory is defined as the result of summing all the planar diagrams.

The idea of a duality between strings and gauge theories was developed further in [3] and led to the AdS/CFT correspondence. As is well known, D-branes are solitonic objects in superstring theory which admit a gauge/gravity low energy interpretation. This is because the low energy dynamic of massless open string states on a Dp brane is, at first order, a $(p+1)$-dimensional supersymmetric gauge theory, and in the closed string channel a Dp brane is a solution of the low energy supergravity solution in presence of a $C_{p}$ Rammond-Rammond $p$-form. The Yang-Mills coupling constant is related to the string coupling by $g_{Y M} \sim g_{s} l_{s}^{p-3}$. This suggests that it is possible to make gauge theory calculations from supergravity solutions and this is indeed one of the motivations of the AdS/CFT correspondence [3]. The original statement of AdS/CFT is that $\mathcal{N}=4$ super Yang-Mills theory is dual to type IIB strings in $A d S^{5} \times S^{5}$. In fact $S U(N)$ $\mathcal{N}=4$ super Yang-Mills is the field theory on $N$ D3 branes at low energies, and the near horizon limit of these branes is $A d S^{5} \times S^{5}$. In addition, for the specific value $p=3$, we have that $g_{Y M} \sim g_{s}$ and one can take the limit $l_{s} \rightarrow 0$ and trust in the supergravity approximation. For $\mathcal{N}=4$ super Yang-Mills theory the beta function vanish at all orders and is therefore conformally invariant, which means that the coupling constant is not renormalized. This is reflected in the $A d S_{5}$ factor of conjectured dual. The theory is also scale invariant quantum mechanically. As a consequence of the conformal symmetry the number of supercharges of the super Yang-Mills side is 32 , the same than IIB superstrings in $A d S^{5} \times S^{5}$. The supergroups of both theories are the same. Besides, both sides contains two parameters. 
For the Yang-Mills theory they are $g_{Y M}$ and $N$ and for the superstring side they are the string tension $R^{2} / \alpha$ and $g_{s}$. In addition to the identification $g_{Y M} \sim g_{s}$ the AdS/CFT conjecture implies that the t' Hooft coupling is given by $\lambda=R^{4} / \alpha^{2}$.

The gravity description of string theory, which occurs when the size of the graviton is much less than the radius of the space, corresponds to the limit $\lambda \gg 1$. In these limits non-perturbative phenomena of the super YangMills side can be analysed. This implies that the AdS/CFT correspondence relates the week coupling limit of one of the theories to the strong coupling of the other and vice versa, which makes it a powerful tool in order to study strongly coupled regimes in gauge theories. A precise statement of the correspondence was developed further in [4], where it was stated that composite operators of the form $O_{i_{1} \ldots i_{k}}(x)=\operatorname{Tr}\left(\phi_{i_{1}} \ldots \phi_{i_{1}}\right)$ mix and are renormalized, therefore they acquire anomalous dimensions. These dimensions are identified with the energy eigenstates of IIB strings over $A d S_{5} \times S^{5}$.

A more recent advance in understanding AdS/CFT is the BMN correspondence, in which the anomalous dimensions of large $R$-operators were related to the spectrum of string theory on the $p p$-wave limit of $A d S_{5} \times S^{5}[6]$. This idea was refined in [7] by stating that gauge theory operators with large spin are dual to strings rotating in the AdS space. The main observation providing this identification is that, for strings rotating in the AdS space, the difference between the energy and the spin of the configuration depends logarithmically on the spin. This logarithmic dependence is characteristic of the anomalous dimensions of the twist operators of the gauge theory. There is evidence about that the logarithmic dependence does not acquire corrections if we go from the perturbative to the strong coupling regime in the t' Hooft coupling [8].

Although $\mathcal{N}=4$ super Yang-Mills cannot be considered as a realistic theory, the AdS/CFT correspondence could be an useful tool in realistic calculations. This is because the finite temperature version of $\mathcal{N}=4 \mathrm{SYM}$ has certain analogies with realistic elementary particle models, although the zero temperature version has not (see for instance [17]). Also, the purely gravitational aspects of this correspondence are related to a wide variety of problems in differential geometry (see for instance [14]). Nevertheless, it is of interest to generalize this duality to other types of theories. This could be for instance theories with less number of supercharges than 32 $[15,16]$, or to consider non-conformal field theories duals [5], such as the Klebanov-Strassler ones [9]. For conformal field theories, the AdS/CFT correspondence has been generalized to the holographic principle [4], in which is stated that any $A d S$ vacuum of string or $M$ theory define a conformal field theory. In the case of $A d S_{5} \times X_{5}$, being $X_{5}$ an Einstein manifold, the 
central charge of the conformal field theory is, in the large $N$ limit, inversely proportional to the volume of $X_{5}$ [18]. The holographic principle permits to consider gauge/string duals with less number of supersymmetries, and have been generalized for 11-dimensional backgrounds of the form $A d S_{4} \times X_{7}$, which are duals to three-dimensional superconformal field theories [4].

The holographic principle renewed the interest in constructing five- and seven-dimensional Einstein manifolds and in particular those admitting at least one conformal Killing spinor. The number of such spinors will be related to the number of supersymmetries of the conformal field theory. This leads to consider weak $G_{2}$ holonomy spaces, Einstein-Sasaki spaces and tri-Sasaki ones. Several examples have been constructed for instance in [31-44] and there have been certain success for finding gauge/gravity duals corresponding to these backgrounds [45-62].

A new step for finding gauge/gravity duals with less number of supersymmetries was achieved in [12], where it was considered a three-parameter deformations of $\mathcal{N}=4$ super Yang-Mills superpotential that preserves $\mathcal{N}=1$ supersymmetry [13] (see also [11]). These deformations are called $\beta$ deformations. The original superpotential of the theory $W=\operatorname{Tr}\left[\left[\Phi_{1}, \Phi_{2}\right], \Phi_{3}\right]$ is transformed as

$$
\begin{aligned}
\operatorname{Tr}\left(\Phi_{1} \Phi_{2} \Phi_{3}-\Phi_{1} \Phi_{3} \Phi_{2}\right) \rightarrow & h \operatorname{Tr}\left(e^{i \pi \beta} \Phi_{1} \Phi_{2} \Phi_{3}-e^{-i \pi \beta} \Phi_{1} \Phi_{3} \Phi_{2}\right) \\
& +h^{\prime} \operatorname{Tr}\left(\Phi_{1}^{3}+\Phi_{2}^{3}+\Phi_{3}^{3}\right),
\end{aligned}
$$

$h, h^{\prime}, \beta$ being complex parameters, satisfying one condition by conformal invariance. One election could be $h^{\prime}=0$. Besides the $U(1)_{R}$ symmetry, there is a $U(1) \times U(1)$ global symmetry generated by

$$
\begin{array}{ll}
U(1)_{1}: & \left(\Phi_{1}, \Phi_{2}, \Phi_{3}\right) \rightarrow\left(\Phi_{1}, e^{i \varphi_{1}} \Phi_{2}, e^{-i \varphi_{1}} \Phi_{3}\right), \\
U(1)_{2}: & \left(\Phi_{1}, \Phi_{2}, \Phi_{3}\right) \rightarrow\left(e^{-i \varphi_{2}} \Phi_{1}, e^{i \varphi_{2}} \Phi_{2}, \Phi_{3}\right),
\end{array}
$$

which leaves the superpotential and the supercharges invariant. Therefore there is a two-dimensional manifold of $\mathcal{N}=1$ CFT with a torus symmetry. It was found in [12] that the $U(1) \times U(1)$ action is realized in gravity part as an isometry. The effect of the $\gamma$-deformation of $\mathcal{N}=4$ super Yang-Mills induce in the gravity dual the simple transformation

$$
\tau \equiv B+i \sqrt{g} \longrightarrow \tau \longrightarrow \tau^{\prime}=\frac{\tau}{1+\gamma \tau}
$$

where $\sqrt{g}$ is the volume of the two torus [12]. The transformation (1.3) indeed comes from a known solution generating-technique explained in [10].

The transformations (1.3) are not the full $S L(2, R)$ transformations. Indeed (1.3) is the subgroup of $S L(2, R)$ for which $\tau \rightarrow 0$ implies that $\tau^{\prime} \rightarrow 0$. In fact, from (1.3) it follows that $\tau_{\gamma}=\tau+o\left(\tau^{2}\right)$ for small $\tau$. Transformations 
with these properties are the only possible ones mapping a ten-dimensional geometry which is non-singular to a new one also without singularities. The reason is that the only points where a singularity can be introduced by performing an $S L(2, R)$ transformation is where the two torus shrinks to zero size. This shrink happens when $\tau^{\prime} \rightarrow 0$ but for $\gamma$ transformations, this implies that $\tau \rightarrow 0$. Therefore, if the original metric was non-singular, then the deformed metric is also non-singular [12]. The transformation (1.3) is the result of doing a T-duality on one circle, a change of co-ordinates, followed by another T-duality. This is another reason for which it can be interpreted as a solution-generating technique [10]. It has been applied recently in order to find several deformed backgrounds in [63-65], together with an analysis of their gauge field theory duals.

Sketch of the present work. In the present work we construct an infinite family of tri-Sasaki metrics in seven dimensions and we find that all these metrics admit a squashed version which is of weak $G_{2}$ holonomy. The idea behind this construction is simple. Our starting point is the Swann hyperKahler metrics [79], which are fibrations over quaternion Kahler metrics $g_{4}$ of the form

$$
g_{s}=|u|^{2} g_{4}+|d u+\omega u|^{2},
$$

$u$ being a certain quaternion co-ordinate and $\omega$ an imaginary quaternion valued one-form associated to the quaternion Kahler space. Under the transformation $u \rightarrow \lambda u$ these metrics are scaled by a factor $g_{s} \rightarrow \lambda^{2} g_{s}$, thus are conical and define a family of tri-Sasaki metrics. We find the explicit expression for this family, which is composed of fibrations over quaternion Kahler spaces. The six-dimensional space formed by the orbits of the Reeb vector is indeed well known, it is the twistor space $Z$ associated to the quaternion Kahler-base. The resulting reduced metric is the natural Kahler-Einstein metric defined over $Z$ [22]. This result is presented in Proposition 2.1 of Section 2.

We show that if the quaternion Kahler base is $\mathrm{CP}(2)$, the resulting triSasaki metric is $N(1,1)_{I}$, which is known to admit a squashed version $N(1,1)_{I I}$ of weak $G_{2}$ holonomy [34]. Guided by this result, we find that the squashed version of any of the tri-Sasaki metrics that we are presenting are of weak $G_{2}$ holonomy. They are indeed the weak $G_{2}$ holonomy metrics defined by the exactly conical family of Bryant-Salamon $\operatorname{Spin}(7)$ metrics [23].

We then manage to find tri-Sasaki (and weak $G_{2}$ holonomy) examples which are locally $T^{3}$ fibrations. These seven-dimensional Einstein metrics are fibred over certian quaternion Kahler orbifolds, the quaternion Kahler limit of the euclidean AdS-Kerr-Newman-Taub-Nut metrics. These fourmetrics itself corresponds to a Wick rotation of the Minkowski 
Plebanski-Demianski metric [81] and were considered in several contexts [82-87]. In some limiting cases for the parameters, the euclidean versions tend to $S^{4}$ or $\mathrm{CP}(2)$, which are smooth. We also consider the most general quaternion Kahler $T^{2}$ fibrations, which were found in [95]. The presence of orbifold singularities is of interest in the context of AdS/CFT correspondence, because they lead to situations generalizing those analysed by Mandal et al. in [76].

We then lift these seven-metrics to supergravity solutions possessing near horizon limit $A d S_{4} \times X_{7}, X_{7}$ being a tri-Sasaki or weak $G_{2}$ holonomy space. We also construct solutions which does not have this horizon limit. In the manifold limit $S^{4}$ of the quaternion Kahler base we analyse rotating membrane configurations and we reproduce the logarithmic behaviour of $\mathrm{E}-\mathrm{S}$, thus these configurations are dual to the "twist" operators of the dual conformal field theory. The methods we use are analogous to those appearing in [19].

We also consider the effect of the $S L(2, R)$ deformation and construct new supergravity solutions, the deformed ones. We reproduce the logarithmic behaviour for the deformed background corresponding to $S^{4}$.

For completeness, we discuss another type of Kahler-Einstein example that is present in the literature and present some Calabi-Yau metrics fibred over non-symmetric Kahler spaces, by using the methods developed in $[68,69]$.

\section{Conical internal spaces}

A wide variety of supergravity backgrounds can be constructed as fibrations over Ricci flat conical metrics. An $n$-dimensional manifold $X_{n}$ develops a conical singularity if and only if it is possible to find a co-ordinate system with a co-ordinate $r$ for which the metric has the local form

$$
g_{n}=d r^{2}+r^{2} g_{n-1},
$$

$g_{n-1}$ being a metric tensor independent on $r$. The metric $g_{n}$ will be singular at $r=0$, except if $g_{n-1}$ is the round $n-1$ dimensional sphere. Any metric $g_{n}$ of the form (2.1) is called a cone over $g_{n-1}$. There also exist metrics taking the form (2.1) for large values of $r$, such metrics are called asymptotically conical. If the conical metric $g_{n}$ is Ricci-flat, i.e., its Ricci tensor satisfies $R_{i j}=0$, then $g_{n-1}$ is Einstein, which means that its Ricci tensor is given by $R_{a b} \sim g_{a b}$. In this section we discuss the geometrical properties of such cones and construct a large family. The geometrical objects that enter in this 
construction are Kahler-Einstein, Einstein-Sasaki, tri-Sasaki, hyperKahler, weak $G_{2}$ and $\operatorname{Spin}(7)$ holonomy spaces. We use the word space instead of manifold in order to keep open the possibility of constructing spaces admitting orbifold or other type of singularities. The reader who is familiar with these concepts can jump to the the last two subsections, in particular to Proposition 2.1.

\section{$2.1 \operatorname{Spin}(7)$ and weak $G_{2}$ holonomy}

A weak $G_{2}$ holonomy space $X_{7}$ is a seven-dimensional space with a metric $g_{7}$ and admitting at least one conformal Killing spinor, i.e., a spinor satisfying $D_{j} \eta \sim \lambda \eta$ [107]. Here $\lambda$ is a constant and $D_{i}$ is the covariant derivative in spinor representation, which is defined by $D_{i}=\partial_{i}+\omega_{i a b} \gamma^{a b}$. The oneform $\omega_{a b}$ is the spin connection on $X_{7}$ defined by the first Cartan equation with zero torsion and $\gamma^{a b}$ is the antisymmetric product of Dirac $\gamma^{a}$ matrices in seven dimensions. If the constant $\lambda$ is zero, then the spinor will be covariantly constant and it will be preserved after parallel transport along any closed curve. This means that the holonomy will be in $G_{2}$, which is the subgroup of $S O(7)$ which possess a one-dimensional invariant subspace. The reduction of the holonomy to $G_{2}$ is equivalent to the existence of a $G_{2}$ invariant three-form

$$
\Phi=c_{a b c} e^{a} \wedge e^{b} \wedge e^{c}
$$

which is covariantly constant, i.e., $\nabla_{X} \Phi=0$ for any vector field $X$. We denote as $e^{a}$ seven-soldering forms for which the metric is diagonal, i.e., $g_{7}=\delta_{a b} e^{a} \otimes e^{b}$ and $c_{a b c}$ are the octonion multiplication constants. This condition holds if and only if $\Phi$ and its dual $* \Phi$ are closed. Instead for a weak $G_{2}$ holonomy space the existence of a conformal Killing vector is equivalent to the condition $d \Phi=\lambda * \Phi$. Such spaces have generically $S O(7)$ holonomy and there exists a frame for which the spin connection $\omega_{a b}$ satisfies

$$
\omega_{a b}=\frac{c_{a b c d}}{2} \omega_{c d}-\lambda c_{a b c} e^{c},
$$

$c_{a b c d}$ being the dual octonion constants. The last condition implies that a weak $G_{2}$ holonomy manifold is always Einstein, i.e., $R_{i j}=\lambda g_{i j}$. In the limit $\lambda \rightarrow 0$ the space will be Ricci flat. This is the case for a $G_{2}$ holonomy space or for any euclidean space admitting covariantly constant Killing spinors.

Spaces with holonomy in $\operatorname{Spin}(7) \in S O(8)$ are eight-dimensional and also admitting one covariantly constant Killing spinor, thus are Ricci flat. Similar to its $G_{2}$ counterpart, they are characterized by a closed $\operatorname{Spin}(7)$ invariant 
four-form

$$
\Phi_{4}=\widetilde{c}_{a b c d} e^{a} \wedge e^{b} \wedge e^{c} \wedge e^{d} .
$$

We are in the middle dimension here and $\Phi_{4}=* \Phi_{4}$. Here $\widetilde{c}_{a b c d}$ are constants related to the octonion constants and from its values it follows that $\Phi_{4}$ can be reexpressed as

$$
\Phi_{4}=e^{8} \wedge \Phi+* \Phi
$$

$\Phi$ being a $G_{2}$ invariant three-form constructed with the seven remaining soldering forms. The expression (2.2) is the origin of a correspondence between conical $\operatorname{Spin}(7)$ holonomy metrics of cohomogeneity one and weak $G_{2}$ holonomy ones. More precisely, any of such $\operatorname{Spin}(7)$ metrics is of the form

$$
g_{8}=d r^{2}+r^{2} g_{7}
$$

$g_{7}$ being a metric of weak $G_{2}$ holonomy and conversely, for any weak $G_{2}$ holonomy metric $g_{7}$ the cone $g_{8}$ will be of $\operatorname{Spin}(7)$ holonomy. This can be seen as follows. Let us consider the choice of the frame $\tilde{e}^{a}$ given by

$$
\tilde{e}^{8}=d r, \quad \tilde{e}^{a}=-\frac{\lambda}{4} r e^{a},
$$

$e^{a}$ being seven soldering forms corresponding to $g_{7}$. In principle there is an $S O(8)$ freedom to choose our frame, but if the element $\tilde{e}^{8}$ is fixed as $\tilde{e}^{8}=d r$, then there it remains an $S O(7)$ freedom only. The first Cartan structure

$$
d \tilde{e}^{a}+\tilde{\omega}^{a b} \wedge \tilde{e}^{b}=0
$$

gives the decomposition

$$
\omega^{a b}=\tilde{\omega}^{a b}, \quad \tilde{\omega}^{8 a}=\frac{\lambda}{4} e^{a},
$$

$\omega_{a b}$ being the spin connection for the seven-dimensional part. Let us assume that the form $\hat{\Phi}$ constructed with (2.3) is closed, this is what we mean about cohomogeneity one. Then (2.3) and (2.2) give

$$
\begin{aligned}
\widetilde{\Phi} & =\left(\frac{\lambda r}{4}\right)^{3} d r \wedge \Phi+\left(\frac{\lambda r}{4}\right)^{4} * \Phi, \\
d \widetilde{\Phi} & =-\left(\frac{\lambda r}{4}\right)^{3} d r \wedge(d \Phi-\lambda * \Phi)+\left(\frac{\lambda r}{4}\right)^{4} d * \Phi,
\end{aligned}
$$

where $\Phi$ and $* \Phi$ are the usual seven-dimensional three- and four-forms constructed with the frame $e^{a}$. It is directly seen from (2.8) that $\operatorname{Spin}(7)$ holonomy condition, namely $d \widetilde{\Phi}=0$ is equivalent to weak $G_{2}$ holonomy of 
the seven-dimensional base space, i.e., to the condition

$$
d \Phi-\lambda * \Phi=0, \quad d * \Phi=0 .
$$

The converse of this statement is also true. Indeed, equations (2.3) are equivalent to

$$
\tilde{\omega}^{a b}=\frac{1}{2} c_{a b c d} \tilde{\omega}^{c d},
$$

which is the eight-dimensional self-duality condition implying the reduction of the holonomy to a subgroup of $\operatorname{Spin}(7)$. This is the one-to-one correspondence we wanted to show [29] (see also [30]).

For applications to marginal deformations of field theories it is needed to focus on metrics with weak $G_{2}$ holonomy admitting $T^{3}$ actions. Examples are the Aloff-Wallach spaces $N(k, l)=\frac{S U(3)}{U(1)}$, which possess two different metrics $N(k, l)_{I}$ and $N(k, l)_{I I}$. Except for $N(1,1)_{I}$, which is tri-Sasaki, the remaining metrics are of weak $G_{2}$ holonomy. For $N(1,1)$ we have the isometry group $S U(3) \times S O(3)$ while for the other cases we have $S U(3) \times$ $U(1)$. Another example is the squashed seven sphere $S O(5) \times \frac{S O(3)}{S O(3)} \times S O(3)$ with isometry group $S O(5) \times S O(3)$. If the manifold is homogeneous, i.e., if $X_{7}$ is of the form $\frac{G}{H}$ then it will be one of this type, see [32,33] for a detailed discussion. Our aim is to construct a more large class of weak $G_{2}$ holonomy manifolds admitting a $T^{3}$ action, not necessarily homogeneous.

\subsection{Einstein-Sasaki and Kahler-Einstein spaces}

As in the previous subsection, let us consider an eight-dimensional space $X_{8}$ endowed with a metric $g_{8}$ and with holonomy in $\operatorname{Spin}(7)$. If $X_{8}$ possess two Killing spinors instead of one, then the holonomy will be reduced further to $S U(4) \in \operatorname{Spin}(7)$. In fact, $S U(4)$ is the subgroup of $S O(8)$ with a two-dimensional invariant subspace. As is well known, any $2 n$-dimensional metric with holonomy $S U(n)$ is Calabi-Yau, and so is $g_{8}$. If in addition $g_{8}$ is conical, then the seven-dimensional metric $g_{7}$ over which $g_{8}$ is fibred will be called Einstein-Sasaki. This metric will possess two conformal Killing spinors. If there is a third Killing spinor, we have a further reduction of the holonomy to $S p(2) \in S U(4)$. Any $4 n$ dimensional space with holonomy in $S p(n)$ is hyperKahler, in particular $g_{8}$. In this case $g_{7}$ will be called triSasaki because it admits three conformal Killing spinors. We can take this notion as definition, for any value of $n$. Clearly, any tri-Sasaki metric is Einstein-Sasaki and if we are in seven dimensions they will be of weak $G_{2}$ holonomy. Also any hyperKahler metric is Calabi-Yau, and in $d=8$ they will be $\operatorname{Spin}(7)$ metrics. The converse of these statements are obviously non-true. 
Any Calabi-Yau space is Ricci flat due to the presence of Killing spinors, and is also Kahler. A Kahler structure over a space $X_{2 n}$ is defined by a doublet $\left(g_{2 n}, I\right)$ composed by an even dimensional metric $g_{2 n}$, a $(1,1)$ antisymmetric tensor $I$ such that $I^{2}=-I$ which is covariantly constant, i.e., $\nabla_{X} I=0 \nabla$ being the Levi-Civita connection and for which the metric is quaternion Hermitian (which means that $g_{2 n}(I X, I Y)=-g_{2 n}(X, Y)$ for any pair of vector fields $X, Y$ of the tangent space at a given point). From the antisymmetry of $I$ it follows that the $(2,0)$ tensor with components $\Omega(X, Y)=g_{2 n}(I X, Y)$ is a two-form. The covariance of $I$ implies that $\Omega$ is closed and that $I$ is integrable, i.e., its Nijenhuis tensor vanishes identically. This implies that $X_{2 n}$ is a complex manifold. Sometimes the triplet $\left(g_{2 n}, I, \Omega\right)$ is identified as the Kahler structure in the literature, but only if the properties stated here are all satisfied.

An Einstein-Sasaki space $X_{2 n+1}$ is always odd-dimensional and can be constructed as an $R$ or $U(1)$-fibration over a Kahler-Einstein metric. The local form of their metric is

$$
g_{2 n+1}=(d \tau+A)^{2}+g_{2 n},
$$

$\Omega=d A$ being the Kahler form of the Kahler-Einstein metric $g_{2 n}$. The metric $g_{2 n}$ is assumed to be $\tau$-independent. The vector $\partial_{\tau}$ is Killing, and it is called the Reeb vector. If the orbits of this vector are closed and the action is free, then $X_{2 n}$ is a manifold and the odd-dimensional manifold $X_{2 n+1}$ is regular. If the action has finite isotropy groups then $X_{2 n}$ is an orbifold. In addition, the Einstein condition $R_{i j} \sim g_{i j}$ for $g_{2 n}$ has been shown to be equivalent to $[70]$

$$
\rho=\Lambda \Omega
$$

$\rho=-i \bar{\partial} \partial \log \operatorname{det} g$ being the Ricci form of the metric $g_{2 n}$. The scalar curvature of $g_{2 n}$ is $2 n \Lambda$.

\subsection{Quaternion Kahler and hyperKahler spaces}

A quaternion Kahler space $M$ is an euclidean $4 n$ dimensional space with holonomy group $\Gamma$ included into the Lie group $S p(n) \times S p(1) \subset S O(4 n)[25-$ 28]. This affirmation is non-trivial if $D>4$, but in $D=4$ there is the wellknown isomorphism $S p(1) \times S p(1) \simeq S U(2)_{L} \times S U(2)_{R} \simeq S O(4)$ and so to state that $\Gamma \subseteq S p(1) \times S p(1)$ is equivalent to state that $\Gamma \subseteq S O(4)$. The last condition is trivially satisfied for any oriented space and gives almost no restrictions, therefore the definition of quaternion Kahler spaces should be modified in $d=4$. 
Here we do a brief description of these spaces; more details can be found in the appendix and in the references therein. For any quaternion, there exists three automorphism $J^{i}(i=1,2,3)$ of the tangent space $T M_{x}$ at a given point $x$ with multiplication rule $J^{i} \cdot J^{j}=-\delta_{i j}+\epsilon_{i j k} J^{k}$. The metric $g_{q}$ is quaternion Hermitian with respect to this automorphism, i.e.,

$$
g_{q}(X, Y)=g\left(J^{i} X, J^{i} Y\right),
$$

$X$ and $Y$ being arbitrary vector fields. The reduction of the holonomy to $S p(n) \times S p(1)$ implies that the $J^{i}$ satisfies the fundamental relation

$$
\nabla_{X} J^{i}=\epsilon_{i j k} J^{j} \omega_{-}^{k},
$$

$\nabla_{X}$ being the Levi-Civita connection of $M$ and $\omega_{-}^{i}$ its $S p(1)$ part. As a consequence of hermiticity of $g$, the tensor $\bar{J}_{a b}^{i}=\left(J^{i}\right)_{a}^{c} g_{c b}$ is antisymmetric, and the associated two-form

$$
\bar{J}^{i}=\bar{J}_{a b}^{i} e^{a} \wedge e^{b}
$$

satisfies

$$
d \bar{J}^{i}=\epsilon_{i j k} \bar{J}^{j} \wedge \omega_{-}^{k},
$$

$d$ being the usual exterior derivative. Corresponding to the $S p(1)$ connection we can define the two-form

$$
F^{i}=d \omega_{-}^{i}+\epsilon_{i j k} \omega_{-}^{j} \wedge \omega_{-}^{k} .
$$

For any quaternion Kahler manifold it follows that

$$
\begin{aligned}
R_{-}^{i} & =2 n \kappa \bar{J}^{i}, \\
F^{i} & =\kappa \bar{J}^{i},
\end{aligned}
$$

$\Lambda$ being a certain constant and $\kappa$ the scalar curvature. The tensor $R_{-}^{a}$ is the $S p(1)$ part of the curvature. The last two conditions imply that $g$ is Einstein with non-zero cosmological constant, i.e., $R_{i j}=3 \kappa\left(g_{q}\right)_{i j} R_{i j}$ being the Ricci tensor constructed from $g_{q}$. The $(0,4)$ and $(2,2)$ tensors

$$
\begin{aligned}
& \Theta=\bar{J}^{1} \wedge \bar{J}^{1}+\bar{J}^{2} \wedge \bar{J}^{2}+\bar{J}^{3} \wedge \bar{J}^{3}, \\
& \Xi=J^{1} \otimes J^{1}+J^{2} \otimes J^{2}+J^{3} \otimes J^{3}
\end{aligned}
$$

are globally defined and covariantly constant with respect to the usual LeviCivita connection for any of these spaces. This implies in particular that any quaternion Kahler space is orientable. 
In four dimensions the Kahler triplet $\bar{J}_{2}$ and the one-forms $\omega_{-}^{a}$ are

$$
\begin{aligned}
& \omega_{-}^{a}=\omega_{0}^{a}-\epsilon_{a b c} \omega_{c}^{b}, \quad \bar{J}_{1}=e^{1} \wedge e^{2}-e^{3} \wedge e^{4}, \\
& \bar{J}_{2}=e^{1} \wedge e^{3}-e^{4} \wedge e^{2}, \quad \bar{J}_{3}=e^{1} \wedge e^{4}-e^{2} \wedge e^{3} .
\end{aligned}
$$

In this dimension quaternion Kahler spaces are defined by the conditions (A:16) and (A:15). This definition is equivalent to state that quaternion Kahler spaces are Einstein and with self-dual Weyl tensor.

In the Ricci-flat limit $\kappa \rightarrow 0$ the holonomy of a quaternion Kahler space is reduced to a subgroup of $S p(n)$ and the resulting spaces are hyperKahler. It follows from (A:.16) and (2.9) that the almost complex structures $J_{i}$ are covariantly constant in this case. Also, there exists a frame for which $\omega_{-}^{i}$ goes to zero. In four dimensions this implies that the spin connection corresponding to this frame is self-dual.

\subsection{An infinite tri-Sasaki family in detail}

The results of this section are crucial for the purposes of the present work. For this reason we will make the calculations in detail. As we have stated, any hyperKahler conical metric $g_{8}$ defines a tri-Sasaki metric by means of the formula $g_{8}=d r^{2}+r^{2} g_{7}$. A well-known family of conical hyperKahler metrics are the Swann metrics [79]; these are $4 n$ dimensional metric but we will focus only in the case $d=8$. The metric reads

$$
g_{8}=|u|^{2} g_{q}+\left|d u+u \omega_{-}\right|^{2},
$$

$g_{q}$ being any four-dimensional quaternion Kahler metric. These metrics represent a sort of inversion of the hyperKahler quotient introduced in [98] (see also [106]). In the expression for the metric we have defined the quaternions

$$
u=u_{0}+u_{1} I+u_{2} J+u_{3} K, \quad \bar{u}=u_{0}-u_{1} I-u_{2} J-u_{3} K,
$$

and the quaternion one form

$$
\omega_{-}=\omega_{-}^{1} I+\omega_{-}^{2} J+\omega_{-}^{3} K,
$$

constructed with the anti-self-dual spin connection. The multiplication rule for the quaternions $I, J$ and $K$ is deduced from

$$
I^{2}=J^{2}=K^{2}=-1, \quad I J=K, \quad J I=-K .
$$

The metric $g_{q}$ is assumed to be independent on the co-ordinates $u_{i}$. We easily see that if we scale $u_{0}, u_{1}, u_{2}, u_{3}$ by $t>0$ this scales the metric by a homothety $t$, which means that the metrics (2.13) are conical. Therefore 
they define a family of tri-Sasaki metrics, which we will find now. We first obtain, by defining $\widetilde{u}_{i}=\frac{u_{i}}{u}$ that

$$
\begin{aligned}
\left|d u+u \omega_{-}\right|^{2}= & \left(d u_{0}-u_{i} \omega_{-}^{i}\right)^{2}+\left(d u_{i}+u_{0} \omega_{-}^{i}+\frac{\epsilon_{i j k}}{2} u_{k} \omega_{-}^{j}\right)^{2} \\
= & \left(\widetilde{u}_{0} d u+u d \widetilde{u}_{0}-u \widetilde{u}_{i} \omega_{-}^{i}\right)^{2} \\
& +\left(\widetilde{u}_{i} d u+u d \widetilde{u}_{i}+u \widetilde{u}_{0} \omega_{-}^{i}+u \frac{\epsilon^{i j k}}{2} \widetilde{u}_{j} \omega_{-}^{k}\right)^{2} \\
= & d u^{2}+u^{2}\left(d \widetilde{u}_{0}-\widetilde{u}_{i} \omega_{-}^{i}\right)^{2}+u^{2}\left(d \widetilde{u}_{i}+\widetilde{u}_{0} \omega_{-}^{i}+\frac{\epsilon_{i j k}}{2} \widetilde{u}_{j} \omega_{-}^{k}\right)^{2} \\
& +2 u u_{0} d u\left(d \widetilde{u}_{0}-\widetilde{u}_{i} \omega_{-}^{i}\right)+2 u u_{i} d u\left(d \widetilde{u}_{i}+\widetilde{u}_{0} \omega_{-}^{i}+\frac{\epsilon^{i j k}}{2} \widetilde{u}_{j} \omega_{-}^{k}\right) .
\end{aligned}
$$

It is not difficult to see that the last two terms are equal to

$$
\begin{aligned}
& 2 u u_{0} d u\left(d \widetilde{u}_{0}-\widetilde{u}_{i} \omega_{-}^{i}\right)+2 u u_{i} d u\left(d \widetilde{u}_{i}+\widetilde{u}_{0} \omega_{-}^{i}+\epsilon_{i j k} \widetilde{u}_{i} \widetilde{u}_{k} \omega_{-}^{j}\right) \\
& =\frac{d\left(\widetilde{u}_{i}^{2}\right)}{2}+\frac{\epsilon_{i j k}}{2} \widetilde{u}_{i} \widetilde{u}_{j} \omega_{-}^{k} .
\end{aligned}
$$

But the second term is a product of an antisymmetric pseudotensor with a symmetric expression, thus is zero, and the first term is zero due to the constraint $\widetilde{u}_{i}^{2}=1$. Therefore this calculation shows that

$$
\left|d u+u \omega_{-}\right|^{2}=d u^{2}+u^{2}\left(d \widetilde{u}_{0}-\widetilde{u}_{i} \omega_{-}^{i}\right)^{2}+u^{2}\left(d \widetilde{u}_{i}+\widetilde{u}_{0} \omega_{-}^{i}+\frac{\epsilon_{i j k}}{2} \widetilde{u}_{j} \omega_{-}^{k}\right)^{2} .
$$

By introducing (2.14) into (2.13) we find that $g_{8}$ is a cone over the following metric

$$
g_{7}=g_{q}+\left(d \widetilde{u}_{0}-\widetilde{u}_{i} \omega_{-}^{i}\right)^{2}+\left(d \widetilde{u}_{i}+\widetilde{u}_{0} \omega_{-}^{i}+\frac{\epsilon_{i j k}}{2} \widetilde{u}_{j} \omega_{-}^{k}\right)^{2} .
$$

This is the tri-Sasaki metric we were looking for. By expanding the squares appearing in (2.15) we find that

$$
\begin{aligned}
g_{7}= & g_{q}+\left(d \widetilde{u}_{i}\right)^{2}+\left(\omega_{-}^{i}\right)^{2}+2 \omega_{-}^{1}\left(\widetilde{u}_{0} d \widetilde{u}_{1}-\widetilde{u}_{1} d \widetilde{u}_{0}+\widetilde{u}_{2} d \widetilde{u}_{3}-\widetilde{u}_{3} d \widetilde{u}_{2}\right) \\
& +2 \omega_{-}^{2}\left(\widetilde{u}_{0} d \widetilde{u}_{2}-\widetilde{u}_{2} d \widetilde{u}_{0}+\widetilde{u}_{2} d \widetilde{u}_{1}-\widetilde{u}_{1} d \widetilde{u}_{3}\right) \\
& +2 \omega_{-}^{3}\left(\widetilde{u}_{0} d \widetilde{u}_{3}-\widetilde{u}_{3} d \widetilde{u}_{0}+\widetilde{u}_{1} d \widetilde{u}_{2}-\widetilde{u}_{2} d \widetilde{u}_{1}\right) .
\end{aligned}
$$

But the expression in parenthesis are a representation of the $S U(2)$ MaurerCartan one-forms, which are defined by

$$
\begin{aligned}
\sigma_{1} & =-\left(\widetilde{u}_{0} d \widetilde{u}_{1}-\widetilde{u}_{1} d \widetilde{u}_{0}+\widetilde{u}_{2} d \widetilde{u}_{3}-\widetilde{u}_{3} d \widetilde{u}_{2}\right) \\
\sigma_{2} & =-\left(\widetilde{u}_{0} d \widetilde{u}_{2}-\widetilde{u}_{2} d \widetilde{u}_{0}+\widetilde{u}_{2} d \widetilde{u}_{1}-\widetilde{u}_{1} d \widetilde{u}_{3}\right) \\
\sigma_{3} & =-\left(\widetilde{u}_{0} d \widetilde{u}_{3}-\widetilde{u}_{3} d \widetilde{u}_{0}+\widetilde{u}_{1} d \widetilde{u}_{2}-\widetilde{u}_{2} d \widetilde{u}_{1}\right) .
\end{aligned}
$$


Therefore the metric $(2.15)$ can be re-expressed in simple fashion as

$$
g_{7}=g_{q}+\left(\sigma_{i}-\omega_{-}^{i}\right)^{2} .
$$

This is one of the expressions that we will use along this work.

Let us recall that there exists a co-ordinate system for which the MaurerCartan forms are expressed as

$$
\begin{gathered}
\sigma_{1}=\cos \varphi d \theta+\sin \varphi \sin \theta d \tau, \quad \sigma_{2}=-\sin \varphi d \theta+\cos \varphi \sin \theta d \tau, \\
\sigma_{3}=d \varphi+\cos \theta d \tau .
\end{gathered}
$$

With the help of this co-ordinate we will write (2.17) in a more customary form for tri-Sasaki spaces, namely

$$
g_{7}=(d \tau+H)^{2}+g_{6},
$$

as in (2.6). Here $g_{6}$ a Kahler-Einstein metric with Kahler form $\bar{J}$ and $H$ a one-form such that $d H=2 \bar{J}$. A lengthy algebraic calculation shows that the fibre metric is

$$
\begin{aligned}
\left(\sigma_{i}-\omega_{-}^{i}\right)^{2}= & \left(d \tau+\cos \theta d \varphi-\sin \theta \sin \varphi \omega_{-}^{1}-\cos \theta \sin \varphi \omega_{-}^{2}-\cos \theta \omega_{-}^{3}\right)^{2} \\
& +\left(\sin \theta d \varphi-\cos \theta \sin \varphi \omega_{-}^{1}-\cos \theta \cos \varphi \omega_{-}^{2}+\sin \theta \omega_{-}^{3}\right)^{2} \\
& +\left(d \theta-\sin \varphi \omega_{-}^{2}+\cos \varphi \omega_{-}^{1}\right)^{2}
\end{aligned}
$$

from where we read that

$$
H=\cos \theta d \varphi-\sin \theta \sin \varphi \omega_{-}^{1}-\cos \theta \sin \varphi \omega_{-}^{2}-\cos \theta \omega_{-}^{3} .
$$

The vector $\partial_{\tau}$ is the Reeb vector, and is a Killing vector. The six-dimensional metric

$$
\begin{aligned}
g_{6}= & g_{q}+\left(d \theta-\sin \varphi \omega_{-}^{2}+\cos \varphi \omega_{-}^{1}\right)^{2}, \\
& +\left(\sin \theta d \varphi-\cos \theta \sin \varphi \omega_{-}^{1}-\cos \theta \cos \varphi \omega_{-}^{2}+\sin \theta \omega_{-}^{3}\right)^{2},
\end{aligned}
$$

should be Kahler-Einstein. We will check that this is the case next.

Another deduction of the tri-Sasaki metrics (2.17). We will prove now that the six-dimensional space formed by the orbits of the Reeb vector of the tri-Sasaki family presented before is the twistor space $Z$ associated to the quaternion Kahler base. The resulting reduced metric (2.21) is the natural Kahler-Einstein metric defined over $Z$ [22]. We need first to define what is $Z$. Recall that for any quaternion Kahler space $M$, a linear combination of the almost complex structures of the form $J=\widetilde{v}_{i} J_{i}$ will be also an almost complex structure on $M$. Here $\widetilde{v}^{i}$ denote three "scalar fields" $\widetilde{v}^{i}=v^{i} / v$ being $v=\sqrt{v^{i} v^{i}}$. These fields are assumed to be constant over $M$ and are obviously constrained by $\widetilde{v}^{i} \widetilde{v}^{i}=1$. This means that the bundle of almost complex structures over $M$ is parameterized by points on the two sphere $S^{2}$. 
This bundle is what is known as the twistor space $Z$ of $M$. The space $Z$ is endowed with the metric

$$
g_{6}=\theta_{i} \theta_{i}+g_{q}
$$

where $\theta_{i}=d\left(\widetilde{v}^{i}\right)+\epsilon^{i j k} \omega_{-}^{j} \widetilde{v}^{k}$. The constraint $\widetilde{v}^{i} \widetilde{v}^{i}=1$ implies that the metric $(2.22)$ is six-dimensional. It has been shown that this metric together with the sympletic two-form $[22,24]$

$$
\bar{J}=-\widetilde{u}_{i} \bar{J}_{i}+\frac{\epsilon_{i j k}}{2} \widetilde{v}_{i} \theta_{j} \wedge \theta_{k},
$$

constitutes a Kahler structure. The calculation of the Ricci tensor of $g_{6}$ shows that it is also Einstein, therefore the space $Z$ is Kahler-Einstein. The expressions given next are written for a quaternion Kahler metric normalized such that $\kappa=1$; for other normalizations certain coefficients must be included in (2.23). By parameterizing the co-ordinates $\widetilde{v}_{i}$ in the spherical form

$$
\widetilde{v}_{1}=\sin \theta \sin \varphi, \quad \widetilde{v}_{2}=\sin \theta \cos \varphi, \quad \widetilde{v}_{3}=\cos \theta,
$$

we find that (2.22) is the same as (2.21). The isometry group of the KahlerEinstein metrics is in general $S O(3) \times G, G$ being the isometry group of the quaternion Kahler basis which also preserves the forms $\omega_{-}^{i}$. The $S O(3)$ part is the one which preserves the condition $\widetilde{v}_{i} \widetilde{v}_{i}=1$. Globally the isometry group could be larger.

From the definition of Einstein-Sasaki geometry, it follows directly that the seven-dimensional metric

$$
g_{7}=(d \tau+H)^{2}+g_{6}=(d \tau+H)^{2}+\theta_{i} \theta_{i}+g_{q},
$$

will be Einstein-Sasaki if $d H=2 \bar{J}$, and we need to find an explicit expression for such $H$. Our aim is to show that this form is indeed (2.20). The expression (2.23) needs to be simplified as follows. We have that $\theta_{i}=d\left(\widetilde{v}^{i}\right)+\epsilon^{i j k} \omega_{-}^{j} \widetilde{v}^{k}$. Also by using the condition $\widetilde{v}_{i} \widetilde{v}_{i}=1$ it is found that

$$
\widetilde{v}_{i} \theta_{i}=\widetilde{v}_{i} d \widetilde{v}_{i}+\epsilon^{i j k} \widetilde{v}^{i} \omega_{-}^{j} \widetilde{v}^{k}=\widetilde{v}_{i} d \widetilde{v}_{i}=d\left(\widetilde{v}_{i} \widetilde{v}_{i}\right)=0 .
$$

From the last equality it follows the orthogonality condition $\widetilde{v}_{i} \theta_{i}=0$ which is equivalent to

The last relation implies that

$$
\theta_{3}=-\frac{\left(\widetilde{v}_{1} \theta_{1}+\widetilde{v}_{2} \theta_{2}\right)}{\widetilde{v}_{3}}
$$

$$
\frac{\epsilon_{i j k}}{2} \widetilde{v}_{i} \theta_{j} \wedge \theta_{k}=\frac{\theta_{1} \wedge \theta_{2}}{\widetilde{v}_{3}}=\frac{d \widetilde{v}_{1} \wedge d \widetilde{v}_{2}}{\widetilde{v}_{3}}-d \widetilde{v}_{i} \wedge \omega_{-}^{i}+\frac{\epsilon^{i j k}}{2} \widetilde{v}_{i} \omega_{-}^{j} \wedge \omega_{-}^{k} .
$$

Besides in a quaternion Kahler manifold with $\kappa=1$ we always have

$$
\widetilde{J}_{i}=d \omega_{-}^{i}+\frac{\epsilon^{i j k}}{2} \omega_{-}^{j} \wedge \omega_{-}^{k}
$$


Inserting the last two expressions into (2.23) gives a remarkably simple expression for $\bar{J}$, namely

$$
\bar{J}=-d\left(\widetilde{v}_{i} \omega_{-}^{i}\right)+\frac{d \widetilde{v}_{1} \wedge d \widetilde{v}_{2}}{\widetilde{v}_{3}} .
$$

By using (2.24) it is obtained that

$$
\frac{d \widetilde{v}_{1} \wedge d \widetilde{v}_{2}}{\widetilde{v}_{3}}=-d \varphi \wedge d \cos \theta
$$

With the help of the last expression we find that (2.26) can be rewritten as

$$
\bar{J}=-d\left(\widetilde{v}_{i} \omega_{-}^{i}\right)-d \varphi \wedge d \cos \theta
$$

from where it is obtained directly that the form $H$ such that $d H=\bar{J}$ is [67]

$$
H=-\widetilde{v}_{i} \omega_{-}^{i}+\cos \theta d \varphi
$$

up to a total differential term. By introducing (2.24) into (2.27) we find that $H$ is the same than $(2.20)$, as we wanted to show.

It will be of importance for the purposes of the present work to state these results in a concise proposition.

Proposition 2.1. Let $g_{q}$ be a four-dimensional Einstein space with self-dual Weyl tensor, i.e., a quaternion Kahler space. We assume the normalization $\kappa=1$ for $g_{q}$. Then the metrics

$$
\begin{aligned}
g_{6}= & g_{q}+\left(d \theta-\sin \varphi \omega_{-}^{2}+\cos \varphi \omega_{-}^{1}\right)^{2} \\
& +\left(\sin \theta d \varphi-\cos \theta \sin \varphi \omega_{-}^{1}-\cos \theta \cos \varphi \omega_{-}^{2}+\sin \theta \omega_{-}^{3}\right)^{2}
\end{aligned}
$$

are Kahler-Einstein while

$$
g_{7}=\left(\sigma_{i}-\omega_{-}^{i}\right)^{2}+g_{q}, \quad g_{8}=d r^{2}+r^{2} g_{7}
$$

are tri-Sasaki and hyperKahler, respectively. Here $\omega_{-}^{i}$ is the Sp(1) part of the spin connection and $\sigma_{i}$ are the usual Maurer-Cartan one-forms over $S O(3)$. Moreover the "squashed" family

$$
g_{7}=\left(\sigma_{i}-\omega_{-}^{i}\right)^{2}+5 g_{q}
$$

is of weak $G_{2}$ holonomy.

We will consider the last sentence of this proposition in the next section. In order to complete this section we would like to describe a little more the Swann bundles. Under the transformation $u \rightarrow G u$ with $G: M \rightarrow S U(2)$ 
the $S U(2)$ instanton $\omega_{-}$is gauge transformed as $\omega_{-} \rightarrow G \omega_{-} G^{-1}+G d G^{-1}$. Therefore the form $d u+\omega_{-} u$ is transformed as

$$
\begin{aligned}
d u & +u \omega_{-} \rightarrow d(G u)+\left(G \omega_{-} G^{-1}+G d G^{-1}\right) G u \\
& =G d u+\left(d G+G \omega_{-}-d G\right) u=G\left(d u+u \omega_{-}\right),
\end{aligned}
$$

and it is seen that $d u+\omega_{-} u$ is a well-defined quaternion-valued one-form over the chiral bundle. The metric (2.13) is also well defined over this bundle. Associated to the metric (2.13) there is a quaternion-valued two-form

$$
\widetilde{\bar{J}}=u \bar{J} \bar{u}+\left(d u+u \omega_{-}\right) \wedge \overline{\left(d u+u \omega_{-}\right)},
$$

and it can be checked that the metric (2.29) is Hermitian with respect to any of the components of (2.28). Also

$$
\begin{aligned}
d \widetilde{\bar{J}}= & d u \wedge\left(\bar{J}+d \omega_{-}-\omega_{-} \wedge \omega_{-}\right) \bar{u}+u \wedge\left(\bar{J}+d \omega_{-}-\omega_{-} \wedge \omega_{-}\right) d \bar{u} \\
& +u\left(d \bar{J}+\omega_{-} \wedge d \omega_{-}-d \omega_{-} \wedge \omega_{-}\right) \bar{u} .
\end{aligned}
$$

The first two terms of the last expression are zero due to (A:.16). Also by introducing (A:.16) into the relation (2.10) it is seen that

$$
d \bar{J}+\omega_{-} \wedge d \omega_{-}-d \omega_{-} \wedge \omega_{-}=0,
$$

and therefore the third term is also zero. This means that the metric (2.13) is hyperKahler with respect to the triplet $\widetilde{\bar{J}}$. The Swann metrics have been considered in several contexts in physics, as for instance in [80, 109-112]. It is an important tool also in differential geometry because the quaternion Kahler quotient construction corresponds to hyperKahler quotients on the Swann fibrations.

\subsection{A weak $G_{2}$ holonomy family by squashing}

In [23] there were probably constructed first examples of $\operatorname{Spin}(7)$ holonomy metrics. These examples are fibred over four-dimensional quaternion Kahler metrics defined over manifold $M$. This resembles the Swann metrics that we have presented in (2.13), although the Bryant-Salamon were found first. The anzatz for the $\operatorname{Spin}(7)$ is

$$
g_{8}=g|u|^{2} \bar{g}+f\left|d u+u \omega_{-}\right|^{2},
$$

where $f$ and $g$ are two unknown functions $f\left(r^{2}\right)$ and $g\left(r^{2}\right)$ which will be determined by the requirement that the holonomy is in $\operatorname{Spin}(7)$, i.e., the closure of the associated four-form $\Phi_{4}$. The analogy between the anzatz 
(2.29) and (2.13) is clear, in fact, if $f=g=1$ the holonomy will be reduced to $S p(2)$. A convenient (but not unique) choice for $\Phi_{4}$ is the following:

$$
\begin{aligned}
\hat{\Phi}= & 3 f g\left[\alpha \wedge \bar{\alpha} \wedge \bar{e}^{t} \wedge e+\bar{e}^{t} \wedge e \wedge \alpha \wedge \bar{\alpha}\right] \\
& +g^{2} \bar{e}^{t} \wedge e \wedge \bar{e}^{t} \wedge e+f^{2} \alpha \wedge \bar{\alpha} \wedge \alpha \wedge \bar{\alpha}
\end{aligned}
$$

where $\alpha=d u+u \omega_{-}$. After imposing the condition $d \Phi_{4}=0$ to $(2.29)$ it is obtained a system of differential equations for $f$ and $g$ with solution

$$
\begin{aligned}
& f=\frac{1}{\left(2 \kappa r^{2}+c\right)^{2 / 5}} \\
& g=\left(2 \kappa r^{2}+c\right)^{3 / 5}
\end{aligned}
$$

and the corresponding metric

$$
g_{s}=\left(2 \kappa r^{2}+c\right)^{3 / 5} \bar{g}+\frac{1}{\left(2 \kappa r^{2}+c\right)^{2 / 5}}|\alpha|^{2} .
$$

Spaces defined by (2.31) are the Bryant-Salamon Spin(7) ones. The metrics (2.31) are non-compact (because $|u|$ is not bounded), and asymptotically conical. They will be exactly conical only if $c=0$. This is better seen by introducing spherical co-ordinates for $u$

$$
\begin{aligned}
& u_{1}=|u| \sin \theta \cos \varphi \cos \tau, \\
& u_{2}=|u| \sin \theta \cos \varphi \sin \tau \\
& u_{3}=|u| \sin \theta \sin \varphi \\
& u_{4}=|u| \cos \theta
\end{aligned}
$$

and defining the radial variable

$$
r^{2}=\frac{9}{20}\left(2 \kappa|u|^{2}+c\right)^{3 / 5}
$$

from which we obtain the spherical form of the metric

$$
g=\frac{d r^{2}}{\kappa\left(1-c / r^{10 / 3}\right)}+\frac{9}{100 \kappa} r^{2}\left(1-\frac{c}{r^{10 / 3}}\right)\left(\sigma^{i}-\omega_{-}^{i}\right)^{2}+\frac{9}{20} r^{2} \bar{g}
$$

$\sigma^{i}$ being the left-invariant one-form on $S U(2)$

$$
\begin{aligned}
\sigma_{1} & =\cos \varphi d \theta+\sin \varphi \sin \theta d \tau \\
\sigma_{2} & =-\sin \varphi d \theta+\cos \varphi \sin \theta d \tau \\
\sigma_{3} & =d \varphi+\cos \theta d \tau .
\end{aligned}
$$

In this case it is clearly seen that (2.32) are of cohomogeneity one and thus, by the results presented in the previous section, they define a weak $G_{2}$ holonomy metric. 
Let us fix the normalization $\kappa=1$, as before. Then in the limit $r \gg c$ the following behaviour is found

$$
g \approx d r^{2}+r^{2} \Omega,
$$

$\Omega$ being a seven-dimensional metric asymptotically independent of the co-ordinate $r$, namely

$$
\Omega=\left(\sigma^{i}-\omega_{-}^{i}\right)^{2}+5 g_{q}
$$

In particular the subfamilies of (2.32) with $c=0$ are exactly conical and their angular part is (2.34). The metrics (2.34) are of weak $G_{2}$ holonomy and possesses an $S O(3)$ isometry action associated with the $\sigma^{i}$. If the fourdimensional quaternion Kahler metric also has an isometry group $G$ that preserves the $\omega_{-}^{i}$, then the group is enlarged to $S O(3) \times G$.

\subsection{An instructive test: the case $N(1,1)_{I}$ and $N(1,1)_{I I}$}

It is important to compare the weak $G_{2}$ holonomy metrics (2.34) and the tri-Sasaki metrics (2.17). The only difference between the two metrics is a factor 5 in front of $g_{q}$ in (2.34). Both metrics possess the same isometry group. At first sight it sounds possible to absorb this factor 5 by a simple rescale of the co-ordinates and therefore to conclude that both metrics are the same. But this is not true. We are fixing the normalization $\kappa=1$ in both cases, thus this factor should be absorbed only by a rescaling on the co-ordinates of the fibre. There is no such rescaling. Therefore, due to this factor 5 , both metrics are different. This is what one expected, since they are metrics of different types.

We can give an instructive example to understand why this is so. Let us consider the Fubini-Study metric on $C P(2)$. This metric is Kahler-Einstein and quaternion Kahler simultaneously and there exists a co-ordinate system for it takes the form

$$
g_{f}=2 d \mu^{2}+\frac{1}{2} \sin ^{2} \mu \widetilde{\sigma}_{3}^{2}+\frac{1}{2} \sin ^{2} \mu \cos ^{2} \mu\left(\widetilde{\sigma}_{1}^{2}+\widetilde{\sigma}_{2}^{2}\right) .
$$

We have denoted the Maurer-Cartan one-forms of this expression as $\widetilde{\sigma}_{i}$ in order to not confuse them with the $\sigma_{i}$ appearing in (2.34) and (2.17). The anti-self-dual part of the spin connection is

$$
\omega_{-}^{1}=-\cos \mu \widetilde{\sigma}_{1}, \quad \omega_{-}^{2}=\cos \mu \widetilde{\sigma}_{2}, \quad \omega_{-}^{3}=-\frac{1}{2}(1+\cos \mu) \widetilde{\sigma}_{3} .
$$


The two metrics that we obtain by use of (2.34) and (2.17) are

$$
\begin{aligned}
g_{7}= & 2 b d \mu^{2}+\frac{1}{2} \sin ^{2} \mu \widetilde{\sigma}_{3}^{2}+b \frac{1}{2} \sin ^{2} \mu \cos ^{2} \mu\left(\widetilde{\sigma}_{1}^{2}+b \widetilde{\sigma}_{2}^{2}\right)+\left(\sigma_{1}+\cos \mu \widetilde{\sigma}_{1}\right)^{2} \\
& +\left(\sigma_{2}-\cos \mu \widetilde{\sigma}_{2}\right)^{2}+\left(\sigma_{3}+\frac{1}{2}(1+\cos \mu) \widetilde{\sigma}_{3}\right)^{2} .
\end{aligned}
$$

If (2.34) and (2.17) are correct, then $b=1$ corresponds to a tri-Sasaki metric and $b=5$ to a weak $G_{2}$ holonomy one. This is true. Locally these metrics are the same as that of $N(1,1)_{I}$ and $N(1,1)_{I I}$ given in [34], which are known to be tri-Sasaki and weak $G_{2}$. We see therefore that this number five in front of the quaternion Kahler metric is relevant and changes topological properties of the metric (such as the number of conformal Killing spinors).

\section{Examples of quaternion Kahler manifolds and orbifolds}

The tri-Sasaki and weak $G_{2}$ holonomy spaces presented in Proposition 2.1 are fibred over quaternion Kahler spaces in four dimensions. Such spaces can be extended to a wide variety of supergravity solutions. We are interested in supergravity solutions with three commuting Killing vectors. In this case the $S L(2, R)$ deformation technique described in [12] can be applied, which in many cases corresponds to marginal deformations of the field theory duals. The Reeb vector is clearly one of the isometries, and by inspection of the formulae of Proposition 2.1 it is seen that three commuting Killing vectors will be obtained if the quaternion Kahler base possesses two commuting isometries which also preserve the one-form, $\omega_{-}^{i}$. We will refer to these spaces as toric quaternion Kahler spaces. In this section we describe a large class of such spaces.

\subsection{Quaternion Kahler limit of AdS-Kerr-Newman-Taub-Nut}

The spaces that we will present next are obtained by a Wick rotation of the Plebanski and Demianski solution [81] and have been discussed in detail in [82-87]. After taking certain scaling limit of the parameters, the distance element becomes

$$
\begin{aligned}
g_{q}= & \frac{x^{2}-y^{2}}{P} d x^{2}+\frac{x^{2}-y^{2}}{Q} d y^{2}+\frac{P}{x^{2}-y^{2}}\left(d \alpha+y^{2} d \beta\right)^{2} \\
& +\frac{Q}{x^{2}-y^{2}}\left(d \alpha+x^{2} d \beta\right)^{2}
\end{aligned}
$$


$P(x)$ and $Q(y)$ being polynomials of the form

$$
P(x)=q-2 s x-t x^{2}-\kappa x^{4}, \quad Q(y)=-P(y),
$$

$(q, s, t, \kappa)$ being four parameters. These expressions can be rewritten as

$$
\begin{aligned}
& P(x)=-\kappa\left(x-r_{1}\right)\left(x-r_{2}\right)\left(x-r_{3}\right)\left(x-r_{4}\right), \\
& Q(y)=-P(y), \quad r_{1}+r_{2}+r_{3}+r_{4}=0,
\end{aligned}
$$

the last condition comes from the fact that $P(x)$ contains no cubic powers of $x$. The two commuting Killing vectors are $\partial_{\alpha}$ and $\partial_{\beta}$.

The metric (3.1) is invariant under the transformation $x \leftrightarrow y$. The transformations $x \rightarrow-x, y \rightarrow-y, r_{i} \rightarrow-r_{i}$ are also a symmetry of the metric. In addition the symmetry $(x, y, \alpha, \beta) \rightarrow\left(\lambda x, \lambda y, \frac{\alpha}{\lambda}, \frac{\beta}{\lambda^{3}}\right), r_{i} \rightarrow \lambda r_{i}$ can be used in order to put one parameter equal to one, so there are only three effective parameters here. The domains of definition are determined by

$$
\left(x^{2}-y^{2}\right) P(x) \geq 0, \quad\left(x^{2}-y^{2}\right) Q(y) \geq 0 .
$$

The anti-self-dual part of the spin connection is

$$
\begin{aligned}
& \omega_{-}^{1}=\frac{\sqrt{P Q}}{y-x} d \beta, \quad \omega_{-}^{3}=\frac{1}{x-y}\left(\sqrt{\frac{Q}{P}} d x+\sqrt{\frac{P}{Q}} d y\right), \\
& \omega_{-}^{2}=-\kappa(x-y) d \alpha+\frac{1}{x-y}\left(q-s(x+y)-t x y-\kappa x^{2} y^{2}\right) d \beta,
\end{aligned}
$$

(see for instance [85]). We will need (3.3) in the following.

The metrics (3.1) are the self-dual limit of the AdS-Kerr-NewmannTaub-Nut solutions, the last ones correspond to the polynomials

$$
P(x)=q-2 s x-t x^{2}-\kappa x^{4}, \quad Q(y)=-q+2 s^{\prime} x+t x^{2}+\kappa x^{4},
$$

and are always Einstein. But the self-duality condition holds if and only if $s^{\prime}=s$, as in (3.2). We will be concerned with this limit in the following, because it is the one which is quaternion Kahler. If we define the new co-ordinates

$$
\begin{gathered}
y=\widetilde{r}, \quad x=a \cos \widetilde{\theta}+N, \\
\alpha=t+\left(\frac{N^{2}}{a}+a\right) \frac{\widetilde{\phi}}{\Xi}, \quad \beta=-\frac{\widetilde{\phi}}{a \Xi},
\end{gathered}
$$

where we have introduced the parameters

$$
\begin{gathered}
\Xi=1-\kappa a^{2}, \quad q=-a^{2}+N^{2}\left(1-3 \kappa a^{2}+3 \kappa N^{2}\right), \\
s=N\left(1-\kappa a^{2}+4 N^{2}\right), \quad t=-1-\kappa a^{2}-6 \kappa N^{2},
\end{gathered}
$$


then the functions $P$ and $Q$ are expressed as

$$
\begin{aligned}
& P=-a^{2} \sin ^{2} \tilde{\theta}\left[1-\kappa\left(4 a N \cos \tilde{\theta}+a^{2} \cos ^{2} \widetilde{\theta}\right)\right], \\
& Q=-\widetilde{r}^{2}-N^{2}+2 s^{\prime} \widetilde{r}+a^{2}+\kappa\left(\widetilde{r}^{4}-a^{2} \widetilde{r}^{2}-6 \widetilde{r}^{2} N^{2}+3 a^{2} N^{2}-3 N^{4}\right),
\end{aligned}
$$

and the metric takes the AdS-Kerr-Newman-Taub-Nut form

$$
\begin{aligned}
g_{q}= & \frac{\sin ^{2} \widetilde{\theta}\left[1-\kappa\left(a^{2} \cos ^{2} \widetilde{\theta}+4 a N \cos \widetilde{\theta}\right)\right]}{R^{2}}\left[a d \widetilde{t}-\frac{r^{2}-a^{2}-N^{2}}{\Xi} d \widetilde{\phi}\right]^{2} \\
& +\frac{\lambda^{2}}{R^{2}}\left[\tilde{t}+\left(\frac{a \sin ^{2} \widetilde{\theta}}{\Xi}-\frac{2 N \cos \widetilde{\theta}}{\Xi}\right) d \widetilde{\phi}\right]^{2} \\
& +\frac{R^{2}}{1-\kappa\left(a^{2} \cos ^{2} \widetilde{\theta}+4 a N \cos \widetilde{\theta}\right)} d \widetilde{\theta}^{2}+\frac{R^{2}}{\lambda^{2}} d r^{2}
\end{aligned}
$$

$R$ and $\lambda$ being defined by

$$
\begin{gathered}
R=\widetilde{r}^{2}-(a \cos \widetilde{\theta}+N)^{2}, \\
\lambda=\widetilde{r}^{2}+N^{2}-2 s^{\prime} \widetilde{r}-a^{2}-\kappa\left(\widetilde{r}^{4}-a^{2} \widetilde{r}^{2}-6 \widetilde{r}^{2} N^{2}+3 a^{2} N^{2}-3 N^{4}\right) .
\end{gathered}
$$

Notice that the self-dual limit corresponds to the choice $s^{\prime}=N\left(1-\kappa a^{2}+\right.$ $\left.4 a N^{2}\right)$ in all the expressions. The parameter $\kappa$ is the scalar curvature of the metric and we fix $\kappa=1$, as we did previously.

These metrics have interesting limits. For $a=0$ and $N$ different from zero becomes the AdS-Taub-Nut solution with local metric

$$
g_{q}=V(\widetilde{r})(\tilde{d \tilde{t}}-2 N \cos \widetilde{\theta} d \widetilde{\phi})^{2}+\frac{d \widetilde{r}^{2}}{V(\widetilde{r})}+\left(\widetilde{r}^{2}-N^{2}\right)\left(d \widetilde{\theta}^{2}+\sin ^{2} \widetilde{\theta} d \widetilde{\phi}^{2}\right)
$$

$V(\widetilde{r})$ being given by

$$
V(\widetilde{r})=\frac{\lambda}{R^{2}}=\frac{1}{\widetilde{r}^{2}-N^{2}}\left(\widetilde{r}^{2}+N^{2}-\left(\widetilde{r}^{4}-6 N^{2} \widetilde{r}^{2}-3 N^{4}\right)-2 s^{\prime} \widetilde{r}\right) .
$$

This metric has been considered in different contexts [82-87]. The parameter $s^{\prime}$ is a mass parameter and $N$ is a nut charge. Both parameters are not independent in the quaternion Kahler limit, in fact the self-duality condition $s^{\prime}=s$ relates them as $s^{\prime}=N\left(1+4 N^{2}\right)$. If the mass were arbitrary then the metric will possess a "bolt", but in this case the metric will possess a "nut", i.e., a zero-dimensional regular fixed point set. The isometry group of (3.5) is enhanced from $U(1) \times U(1)$ to $S U(2) \times U(1)$ in this limit. The anti-self-dual 
part of the spin connection reads

$$
\begin{gathered}
\omega_{-}^{1}=-\sqrt{(\widetilde{r}+N) V(\widetilde{r})} \sin \widetilde{\theta} d \widetilde{\phi}, \quad \omega_{-}^{3}=\sqrt{(\widetilde{r}+N) V(\widetilde{r})} d \widetilde{\theta}, \\
\omega_{-}^{2}=(\widetilde{r}-N) d \widetilde{t}+g(\widetilde{r}) \cos \widetilde{\theta} d \widetilde{\phi}
\end{gathered}
$$

$g(\widetilde{r})$ being defined by

$$
g(\widetilde{r})=\left(\frac{N^{2}(\widetilde{r}-N)+N\left(1+4 N^{2}\right)+\left(1+6 N^{2}\right) \widetilde{r}-2 N \widetilde{r}^{2}}{\widetilde{r}-N}\right) .
$$

By taking the further limit $N=0$, i.e., but switching off the mass and the charge, we obtain the following distance element after introducing the new radius: $\widetilde{r}=\sin \widetilde{\rho}$

$$
g_{q}=\cos ^{2} \widetilde{\rho} d \widetilde{t}^{2}+d \widetilde{\rho}^{2}+\sin ^{2} \widetilde{\rho}\left(d \widetilde{\theta}^{2}+\sin ^{2} \widetilde{\theta} d \widetilde{\phi}^{2}\right) .
$$

The anti-self-dual spin connection takes the simple form

$$
\omega_{-}^{1}=\cos \widetilde{\rho} \sin \widetilde{\theta} d \widetilde{\phi}, \quad \omega_{-}^{2}=\sin \widetilde{\rho} d \widetilde{t}+\cos \widetilde{\theta} d \widetilde{\phi}, \quad \omega_{-}^{3}=\cos \widetilde{\rho} d \widetilde{\theta},
$$

and it follows that we have obtained the metric of the sphere $S^{4}=S O(5) /$ $S O(4)$. If we would choose negative scalar curvature instead, this limit would correspond to the non-compact space $S O(4,1) / S O(4)$. Both cases are maximally symmetric and for this reason this is called the $A d S_{4}$ limit of the AdS-Taub-Nut solution.

The other four-dimensional quaternion Kahler manifold is $\mathrm{CP}(2)$ with the Fubbini-Study metric. This case is obtained by defining the new co-ordinates $\widehat{r}=N(\widetilde{r}-N)$ and $\widetilde{t}=2 N \xi$ and taking the limit $N \rightarrow \infty$. The result, after defining $\widetilde{\rho}=\widehat{r}^{2} / 4\left(1+\widehat{r}^{2}\right)$, is the metric

$$
g_{q}=\frac{\widetilde{\rho}^{2}}{2\left(1+\widetilde{\rho}^{2}\right)^{2}}(d \xi-\cos \widetilde{\theta} d \widetilde{\phi})^{2}+\frac{2 d \widetilde{\rho}^{2}}{\left(1+\widetilde{\rho}^{2}\right)^{2}}+\frac{\widetilde{\rho}^{2}}{2\left(1+\widetilde{\rho}^{2}\right)^{2}}\left(d \widetilde{\theta}^{2}+\sin ^{2} \widetilde{\theta} d \widetilde{\phi}^{2}\right) .
$$

By noticing that $\sigma_{3}=d \xi-\cos \widetilde{\theta} d \widetilde{\phi}$ and that $\sigma_{1}^{2}+\sigma_{2}^{2}=d \widetilde{\theta}^{2}+\sin ^{2} \widetilde{\theta} d \widetilde{\phi}^{2}$ we recognize from (3.7) the Bianchi IX form for the Fubbini-Study metric on $\mathrm{CP}(2)=S U(3) / S U(2)$.

Another possible limit of (3.8) is $N \rightarrow 0$, the result will be the AdS-Kerr Euclidean solution, namely

$$
\begin{aligned}
g_{q}= & \frac{\sin ^{2} \widetilde{\theta}\left(1-a^{2} \cos ^{2} \widetilde{\theta}\right)}{\widetilde{r}^{2}-a^{2} \cos ^{2} \widetilde{\theta}}\left(a d \widetilde{t}-\frac{\widetilde{r}^{2}-a^{2}}{\Xi} d \widetilde{\phi}\right)^{2}+\frac{\widetilde{r}^{2}-a^{2} \cos \widetilde{\theta}^{2}}{1-a^{2} \cos ^{2} \widetilde{\theta}} d \widetilde{\theta}^{2} \\
& +\frac{\widetilde{r}^{2}-a^{2} \cos \widetilde{\theta}^{2}}{\left(\widetilde{r}^{2}-a^{2}\right)\left(1-\widetilde{r}^{2}\right)} d \widetilde{r}^{2}+\frac{\left(\widetilde{r}^{2}-a^{2}\right)\left(1-\widetilde{r}^{2}\right)}{\widetilde{r}^{2}-a^{2} \cos \widetilde{\theta}^{2}}\left(d \widetilde{t}+\frac{a \sin ^{2} \widetilde{\theta}}{\Xi} d \widetilde{\phi}\right)^{2} .
\end{aligned}
$$


The anti-self-dual connection $\omega_{-}^{i}$ is in this case

$$
\begin{aligned}
& \omega_{-}^{1}=-\frac{1}{\widetilde{r}-a \cos \widetilde{\theta}} \sqrt{\left(1-a^{2} \cos \widetilde{\theta}^{2}\right)\left(\widetilde{r}^{2}-a^{2}\right)\left(1-\widetilde{r}^{2}\right) \frac{\sin \widetilde{\theta}}{\Xi} d \widetilde{\phi}} \\
& \omega_{-}^{2}=(\widetilde{r}-a \cos \widetilde{\theta}) d \widetilde{t}+\frac{1}{\widetilde{r}-a \cos \widetilde{\theta}} \frac{W(\widetilde{r}, \widetilde{\theta})}{\Xi} d \widetilde{\phi} \\
& \omega_{-}^{3}=\frac{1}{\widetilde{r}-a \cos \widetilde{\theta}}\left(\sqrt{\frac{\left(\widetilde{r}^{2}-a\right)\left(1-\widetilde{r}^{2}\right)}{1-a^{2} \cos ^{2} \widetilde{\theta}}} d \widetilde{\theta}-\sqrt{\frac{1-a^{2} \cos ^{2} \widetilde{\theta}}{\left(\widetilde{r}^{2}-a^{2}\right)\left(1-\widetilde{r}^{2}\right)}} a \sin \tilde{\theta} d \widetilde{r}\right),
\end{aligned}
$$

where we have defined the function

$$
W(\widetilde{r}, \widetilde{\theta})=\left[(\widetilde{r}-a \cos \widetilde{\theta})^{2}-a+\left(1+a^{2}\right) \widetilde{r} \cos \widetilde{\theta}-a \widetilde{r}^{2} \cos ^{2} \widetilde{\theta}\right] .
$$

The parameter $a$ is usually called rotational parameter, although we have no notion of the rotational black hole in Euclidean signature. The mass parameter $s$ and the nut charge are zero in this case.

\subsection{Toric quaternion Kahler spaces}

We turn now to more general toric quaternion Kahler orbifolds, following [95]. As we have mentioned, in four dimensions quaternion Kahler spaces are the same than Einstein spaces with self-dual Weyl tensor. The self-duality condition is conformally invariant. This means that if a metric $g$ is self-dual, then all the family $[g]$ of metrics obtained by $g$ by conformal transformations is self-dual. The Einstein condition instead is not invariant under conformal transformations.

Let us focus first in the construction of self-dual families with $U(1) \times$ $U(1)$ isometry, the Einstein condition will be considered afterwards. For any representative $g$ of a toric conformal family $[g]$ there exists a co-ordinate system for which the metric is expressed in the Gowdy form

$$
g=g_{a b} d x^{a} d x^{b}+g_{\alpha \beta} d x^{\alpha} d x^{\beta} .
$$

The latin and greek indices take values 1 and 2 . Both $g_{a b}$ and $g_{\alpha \beta}$ are supposed to be independent of the co-ordinates $x^{\alpha}=(\alpha, \beta)$. The Killing vectors are then $\partial_{\alpha}$ and $\partial_{\beta}$ and are commuting, so there is a $U(1) \times U(1)$ action on the manifold. This consideration is local and no assumption about the $U(1)$ actions is made. ${ }^{1}$

\footnotetext{
${ }^{1}$ We are loosely speaking about toric conformal families. If a metric $g$ has two commuting Killing vectors $\partial_{\alpha}$ and $\partial_{\beta}$, then by a conformal transformation $g \rightarrow \Omega^{2} g$ depending on $(\alpha, \beta)$ we will obtain a new metric which is not toric anymore. Along this section the $\Omega \mathrm{s}$
} 
Clearly, the part $g_{a b} d x^{a} d x^{b}$ in (3.11) can be interpreted as a twodimensional metric. By a theorem due to Gauss it is known that every two-dimensional metric is conformally flat. Therefore the anzatz (3.11) can be written as

$$
g=\Omega^{2}\left(d \rho^{2}+d \eta^{2}\right)+g_{\alpha \beta} d x^{\alpha} d x^{\beta},
$$

$g_{\alpha \beta}$ being the functions of the co-ordinates $(\rho, \eta)$ and $\Omega$ a conformal factor with the same co-ordinate dependence. Because self-duality is conformally invariant we can make a conformal transformation to (3.12) and consider the following metric

$$
\bar{g}=\frac{\left(d \rho^{2}+d \eta^{2}\right)}{\rho^{2}}+\bar{g}_{\alpha \beta} d x^{\alpha} d x^{\beta},
$$

without loosing generality. The factor $\rho^{2}$ was introduced by convenience. It is natural to express $\bar{g}_{\alpha \beta} d x^{\alpha} d x^{\beta}$ in terms of certain functions $\bar{A}_{i}$ and $\bar{B}_{i}$ $(i=1,2)$ of $(\rho, \eta)$ as

$$
\bar{g}_{\alpha \beta} d x^{\alpha} d x^{\beta}=\left(\bar{A}_{0} d \theta-\bar{B}_{0} d \varphi\right)^{2}+\left(\bar{A}_{1} d \theta-\bar{B}_{1} d \varphi\right)^{2} .
$$

But this is not the most simplifying form in order to impose the self-duality condition. It is more convenient to write it as

$$
\bar{g}_{\alpha \beta} d x^{\alpha} d x^{\beta}=\frac{\left(A_{0} d \theta-B_{0} d \varphi\right)^{2}+\left(A_{1} d \theta-B_{1} d \varphi\right)^{2}}{\left(A_{0} B_{1}-A_{1} B_{0}\right)^{2}},
$$

in terms of new functions $A_{i}$ and $B_{i}$. The relation with the other functions $\bar{A}_{i}$ and $\bar{B}_{i}$ is given by comparison of the last two expressions. Although considering the anzatz (3.15) could seem non-practical, the self-duality condition became a linear differential system in terms of $A_{i}$ and $B_{i}{ }^{2}$ Therefore

are supposed to be independent on $(\alpha, \beta)$. In this case it is ensured that $\partial_{\alpha}$ and $\partial_{\beta}$ will be the Killing vectors of every metric in $[g]$.

${ }^{2}$ An intuitive argument to see that an anzatz of the form (3.18) could simplify the self-duality condition goes as follows. It is known that for any group of four vector fields $e_{i}$, the Ashtekar et al. equations [92]

$$
\left[e_{1}, e_{2}\right]+\left[e_{3}, e_{4}\right]=0, \quad\left[e_{1}, e_{3}\right]+\left[e_{4}, e_{2}\right]=0, \quad\left[e_{1}, e_{4}\right]+\left[e_{2}, e_{3}\right]=0,
$$

define a self-dual metric $g=\delta_{a b} e^{a} \otimes e^{b}$ (here $e_{i}$ is the dual basis of $e^{a}$ ) called hypercomplex metric. The reader can check that if we select the following soldering forms

$$
e^{1}=d \rho, \quad e^{2}=d \eta, \quad e^{3}=\left(\frac{A_{0} d \theta-B_{0} d \varphi}{A_{0} B_{1}-A_{1} B_{0}}\right), \quad e^{4}=\left(\frac{A_{1} d \theta-B_{1} d \varphi}{A_{0} B_{1}-A_{1} B_{0}}\right)
$$

for the metric (3.18) then the inverse basis take the simple form

$$
\begin{aligned}
& e_{1}=\partial_{\rho}, \quad e_{2}=\partial_{\eta} \\
& e_{3}=B_{1} \partial_{\theta}+A_{1} \partial_{\varphi}, \quad e_{4}=B_{0} \partial_{\theta}+A_{0} \partial_{\varphi},
\end{aligned}
$$

and the Ashtekar conditions became simply Cauchy-Riemann equations

$$
\begin{aligned}
& \left(A_{1}\right)_{\rho}=\left(A_{0}\right)_{\eta}, \quad\left(A_{0}\right)_{\rho}=-\left(A_{1}\right)_{\eta} \\
& \left(B_{1}\right)_{\rho}=\left(B_{0}\right)_{\eta}, \quad\left(B_{0}\right)_{\rho}=-\left(B_{1}\right)_{\eta}
\end{aligned}
$$


it is better to impose the self-duality condition to

$$
\bar{g}=\frac{\left(d \rho^{2}+d \eta^{2}\right)}{\rho^{2}}+\frac{\left(A_{0} d \theta-B_{0} d \varphi\right)^{2}+\left(A_{1} d \theta-B_{1} d \varphi\right)^{2}}{\left(A_{0} B_{1}-A_{1} B_{0}\right)^{2}} .
$$

If also the commuting Killing vectors are surface orthogonal, then the condition $W=* W$ gives the linear system

$$
\begin{aligned}
& \left(A_{0}\right)_{\rho}+\left(A_{1}\right)_{\eta}=A_{0} / \rho, \\
& \left(A_{0}\right)_{\eta}-\left(A_{1}\right)_{\rho}=0,
\end{aligned}
$$

and the same equations for $B_{i}$. This system was found by Joyce in [102]. Surface orthogonality implies that the manifold $M$ corresponding to (3.18) is of the form $M=N \times T^{2}, T^{2}$ being the two-dimensional torus. There exists other examples (as those in the footnote) which are solution of the self-duality conditions but for which this decomposition does not hold.

The linear system (3.19) and (3.20) is simple enough. It is easy to check that (3.20) implies the existence of a potential function $G$ such that

$$
A_{0}=G_{\rho} ; \quad A_{1}=G_{\eta} .
$$

Then (3.19) gives that $G_{\rho \rho}+G_{\eta \eta}=G_{\rho} / \rho$. Inversely we deduce from (3.19) the existence of another potential $V$ such that

$$
A_{0}=-\rho V_{\eta} ; \quad A_{1}=\rho V_{\rho}
$$

and (3.20) gives the Ward monopole equation [71]

$$
V_{\eta \eta}+\rho^{-1}\left(\rho V_{\rho}\right)_{\rho}=0
$$

The relations

$$
G_{\rho}=-\rho V_{\eta} ; \quad G_{\eta}=\rho V_{\rho},
$$

constitute a Backlund transformation allowing to find a monopole $V$ starting with a known potential $G$ or vice versa. The functions $B_{i}$ can be also expressed in terms of another potential functions $G^{\prime}$ and $V^{\prime}$ satisfying the same equations than $V$ and $G$.

The next task is to extract the Einstein representatives of the self-dual families defined in (3.18). This will be automatically quaternion Kahler. In general, to impose the Einstein condition directly to (3.18) could give no results. In order to be general, a transformation $\bar{g} \rightarrow \Omega^{2} \bar{g}$ should be made to the metrics (3.18) and the Einstein condition $R_{i j}=\Lambda g_{i j}$ should be imposed to the transformed metrics. This requirement will fix the factor $\Omega$ and will

which are linear. If (3.14) is used instead, then the resulting system will take a non-linear "look." 
give further relations between $A_{i}$ and $B_{i}$. The result obtained from this condition is that

$$
A_{1} B_{0}-A_{0} B_{1}=\rho\left(A_{0}^{2}+A_{1}^{2}\right)-G A_{0},
$$

and that $\Omega^{2}=G$. From (3.25) it is obtained that $B_{0}=\rho A_{1}+\xi_{0}$ and $B_{1}=$ $G-\rho A_{0}+\xi_{1}$ with $A_{1} \xi_{0}=A_{0} \xi_{1}$. The functions $\xi_{i}$ are determined by the requirement that $B_{i}$ also satisfies the Joyce system (3.19) and (3.20), the result is $\xi_{0}=-\eta A_{0}$ and $\xi_{1}=-\eta A_{1}$. Then it is obtained that

$$
\begin{gathered}
A_{0}=G_{\rho} ; \quad A_{1}=G_{\eta} \\
B_{0}=\eta G_{\rho}-\rho G_{\eta} ; \quad B_{1}=\rho G_{\rho}+\eta G_{\eta}-G .
\end{gathered}
$$

By defining $G=\sqrt{\rho} F$ it follows from the equation $G_{\rho \rho}+G_{\eta \eta}=G_{\rho} / \rho$ that F satisfies

$$
F_{\rho \rho}+F_{\eta \eta}=\frac{3 F}{4 \rho^{2}}
$$

The final expression of the metric is [93]

$$
\begin{aligned}
g_{q}= & \frac{F^{2}-4 \rho^{2}\left(F_{\rho}^{2}+F_{\eta}^{2}\right)}{4 F^{2}} \frac{d \rho^{2}+d \eta^{2}}{\rho^{2}} \\
& +\frac{\left[\left(F-2 \rho F_{\rho}\right) u-2 \rho F_{\eta} v\right]^{2}+\left[-2 \rho F_{\eta} u+\left(F+2 \rho F_{\rho}\right) v\right]^{2}}{F^{2}\left[F^{2}-4 \rho^{2}\left(F_{\rho}^{2}+F_{\eta}^{2}\right)\right]},
\end{aligned}
$$

where $u=\sqrt{\rho} d \alpha, v=(d \beta+\eta d \alpha) / \sqrt{\rho}$ and $F(\rho, \eta)$ is a solution of the equation

$$
F_{\rho \rho}+F_{\eta \eta}=\frac{3 F}{4 \rho^{2}}
$$

on some open subset of the half-space $\rho>0$.

There exists a theorem that insures that a toric quaternion Kahler manifold will always have surface orthogonal Killing vectors [95]. Therefore the metrics (3.28) are the most general toric quaternion Kahler ones. On the open set defined by $F^{2}>4 \rho^{2}\left(F_{\rho}^{2}+F_{\eta}^{2}\right) g$ has positive scalar curvature, whereas $F^{2}<4 \rho^{2}\left(F_{\rho}^{2}+F_{\eta}^{2}\right)-\mathrm{g}$ is self-dual with negative scalar curvature. This is known as the Calderbank-Pedersen metrics.

\subsection{The manifolds of the family: $\mathrm{CP}(2)$ and $S^{4}$}

If we choose a potential $F$ independent on the co-ordinate $\eta$, then it is obtained from (3.29) that it should be of the form $F(\rho)=\rho^{3 / 2}-c \rho^{-1 / 2}$ 
$c$ being a constant. In this case the metric (3.28) will have the explicit form

$$
g_{K}=\frac{\rho^{2}+c}{\left(\rho^{2}-c\right)^{2}}\left(d \rho^{2}+d \eta^{2}+\frac{d \alpha^{2}}{4}\right)+\frac{\rho^{2}}{\left(\rho^{2}-c\right)^{2}\left(\rho^{2}+c\right)}(d \beta+\eta d \alpha)^{2},
$$

and the isometry group will be enlarged by the presence of a new Killing vector $\partial_{\eta}+\beta \partial_{\alpha}$. The action of the isometry group on the co-ordinates is given by

$$
\alpha \rightarrow \alpha+a_{1}, \quad \eta \rightarrow \eta+a_{2}, \quad \beta \rightarrow \beta+a_{3}-a_{2} \alpha
$$

$a_{i}$ being constant parameters. It is worthy to mention that the maximally symmetric quaternion Kahler spaces possess $S O(5)$ or $S O(4,1)$ isometry group, therefore there can be at most two commuting isometries in a fourdimensional quaternion Kahler space. In other words, it is impossible that the third isometry will commute with the others. Indeed the three Killing vectors $T_{i}$ satisfy the three-dimensional Heisenberg algebra

$$
\left[T_{1}, T_{3}\right]=T_{2}, \quad\left[T_{1}, T_{2}\right]=\left[T_{3}, T_{2}\right]=0 .
$$

The isometry group (3.31) preserves the triplet (4.5).

If also $c=0$ then the potential function is $F=\rho^{3 / 2}$ and the expression for the quaternion Kahler metric will be

$$
g_{K}=\frac{1}{\rho^{2}}\left(d \rho^{2}+d \eta^{2}+\frac{d \alpha^{2}}{4}\right)+\frac{1}{\rho^{4}}(d \beta+\eta d \alpha)^{2} .
$$

Let us introduce the two-form

$$
\bar{J}^{\prime}=\frac{d \alpha \wedge d \eta}{2 \rho^{2}}+d\left(\frac{1}{2 \rho^{2}}\right) \wedge(d \beta+\eta d \alpha),
$$

which preserved locally under the action $(3.31)$. The $(1,1)$ tensor $J^{\prime}$ defined through the relation $g_{q}\left(\cdot, J^{\prime} \cdot\right)=\bar{J}^{\prime}$ is an almost complex structure defined over the quaternion Kahler space $M$. The two-form $\bar{J}^{\prime}$ is evidently closed, thus sympletic and it can be expressed as $\bar{J}^{\prime}=d A$ being the one-form $A$ given by

$$
A=-\frac{(d \beta+\eta d \alpha)}{2 \rho^{2}}
$$

By introducing the complex quantities

$$
S=\rho^{2}+i(2 \beta+\eta \alpha)+C \bar{C}, \quad C=i \eta+\frac{1}{2} \alpha,
$$

it follows that the metric (3.33) can be written as

$$
g_{K}=u \otimes \bar{u}+v \otimes \bar{v}
$$

where

$$
u=\frac{1}{\rho} d C, \quad v=\frac{1}{2 \rho^{2}}(d S+C d \bar{C}) .
$$


This is the classical metric of the universal dilaton hypermultiplet [105] and is known to be Kahler with Kahler potential

$$
K=\log (S+\bar{S}-2 C \bar{C}) .
$$

This means that space $M$ is not only sympletic, but Kahler. It is also quaternion Kahler (thus Einstein), therefore is Kahler-Einstein. The form (3.34) is expressed in the co-ordinates $(S, C, \bar{S}, \bar{C})$ as

$$
A=2\left(\frac{d S-d \bar{S}+C d \bar{C}-\bar{C} d C}{S+\bar{S}-2 C \bar{C}}\right) .
$$

By going to the co-ordinate system defined by

$$
z_{1}=\frac{1-S}{1+S}, \quad z_{2}=\frac{2 C}{1+S}
$$

it is recognized that (3.37) is the Bargmann metric

$$
g_{q}=\frac{d z_{1} d \bar{z}_{1}+d z_{2} d \bar{z}_{2}}{1-\left|z_{1}\right|^{2}-\left|z_{2}\right|^{2}}-\frac{\left(z_{1} d \bar{z}_{1}+z_{2} d \bar{z}_{2}\right)\left(\bar{z}_{1} d z_{1}+\bar{z}_{2} d z_{2}\right)}{\left(1-\left|z_{1}\right|^{2}-\left|z_{2}\right|^{2}\right)^{2}},
$$

defined over the unit open ball in $C^{2}$ given by $\left|z_{1}\right|^{2}+\left|z_{2}\right|^{2}<1$, with Kahler potential $K=\log \left(1-\left|z_{1}\right|^{2}-\left|z_{2}\right|^{2}\right)$. This space is topologically equivalent to the homogeneous symmetric space $S U(2,1) / S U(2) \times S U(1)$. By going to spherical co-ordinates

$$
z_{1}=r \cos \frac{u}{2} \exp \left(i \frac{(v+w)}{2}\right), \quad z_{2}=r \sin \frac{u}{2} \exp \left(-i \frac{(v-w)}{2}\right),
$$

with $0<r<1,0<u<\pi, 0<v<2 \pi$ and $0<w<4 \pi$, the Bargmann metric takes the Bianchi IX form

$$
g_{q}=\frac{d r^{2}}{\left(1-r^{2}\right)^{2}}+\frac{r^{2} \sigma_{1}^{2}}{\left(1-r^{2}\right)^{2}}+\frac{r^{2}\left(\sigma_{2}^{2}+\sigma_{3}^{2}\right)}{1-r^{2}}
$$

with manifest $S U(2)$ symmetry.

Let us note that the metrics (3.33) can be extended with no difficulty to an Einstein-Sasaki space in five dimensions by use of (2.6), the local form of such metrics is

$$
g_{e s}=\left[d \tau-\frac{(d \beta+\eta d \alpha)}{2 \rho^{2}}\right]^{2}+\frac{1}{\rho^{2}}\left(d \rho^{2}+d \eta^{2}+\frac{d \alpha^{2}}{4}\right)+\frac{1}{\rho^{4}}(d \beta+\eta d \alpha)^{2},
$$

and is seen that the isometry group (3.31) is an isometry of (3.42). We have three commuting Killing vectors, namely $\partial_{\alpha} \partial_{\beta}$ and $\partial_{\tau}$, and so there is a $T^{3}$ action. 
For the Bargmann metric $F^{2}<4 \rho^{2} F_{\rho}^{2}$ and this means that $\kappa<0$. Thus the construction of tri-Sasaki metrics presented in previous sections cannot be applied to this example. But by making the replacement $z_{i} \rightarrow i z_{i}$ the Kahler potential of the Bargmann metric will be converted into $K=$ $\log \left(1+\left|z_{1}\right|^{2}+\left|z_{2}\right|^{2}\right)$. This is the potential for the Fubbini-Study metric over $\mathrm{CP}(2)$, which is dual to the Bergmann one. This metric is also KahlerEinstein and quaternion Kahler. Its metric tensor and the corresponding potential form $A$ can obtained from the formulae corresponding to the Bargmann metric by making the replacement $z_{i} \rightarrow i z_{i}$. But different from the Bargmann metric, this metric possesses positive scalar curvature and the construction presented in the previous section can be applied. The antiself-dual part of the spin connection of the Fubbini-Study metric is given by

$$
\begin{aligned}
& \omega_{-}^{1}=-\frac{1}{2}\left(\frac{\bar{z}_{2} d z_{1}+z_{2} d \bar{z}_{1}}{\left(1+\left|z_{1}\right|^{2}\right) \sqrt{1+\left|z_{1}\right|^{2}+\left|z_{2}\right|^{2}}}\right), \\
& \omega_{-}^{2}=\frac{i}{2}\left(\frac{\bar{z}_{2} d z_{1}-z_{2} d \bar{z}_{1}}{\left(1+\left|z_{1}\right|^{2}\right) \sqrt{1+\left|z_{1}\right|^{2}+\left|z_{2}\right|^{2}}}\right), \\
& \omega_{-}^{3}=\frac{i}{2}\left(\frac{\bar{z}_{1} d z_{1}-z_{1} d \bar{z}_{1}+z_{2} d \bar{z}_{2}-\bar{z}_{2} d z_{2}}{\left(1+\left|z_{1}\right|^{2}\right)}\right) .
\end{aligned}
$$

From (2.17) the tri-Sasaki metric is obtained

$$
g_{7}=\left(\sigma_{i}-\omega_{-}^{i}\right)^{2}+\frac{d z_{1} d \bar{z}_{1}+d z_{2} d \bar{z}_{2}}{1+\left|z_{1}\right|^{2}+\left|z_{2}\right|^{2}}-\frac{\left(z_{1} d \bar{z}_{1}+z_{2} d \bar{z}_{2}\right)\left(\bar{z}_{1} d z_{1}+\bar{z}_{2} d z_{2}\right)}{\left(1+\left|z_{1}\right|^{2}+\left|z_{2}\right|^{2}\right)^{2}},
$$

which is fibred over the Fubbini-Study metric. Here $\sigma_{i}$ is the one MaurerCartan forms in (2.4) and $\omega_{-}^{i}$ is given in (3.43).

The same procedure can be applied to the sphere $S^{4}$, which, together with $C P(2)$ constitute the unique four-dimensional quaternion Kahler spaces that are manifolds. The corresponding Kahler-Einstein and tri-Sasaki metrics are respectively

$$
\begin{aligned}
g_{6}= & \frac{1}{2} d \Omega_{4}^{2}+\left(d \theta-\sin \varphi A^{2}+\cos \varphi A^{1}\right)^{2} \\
& +\left(\sin \theta d \varphi-\cos \theta \sin \varphi A^{1}-\cos \theta \cos \varphi A^{2}+\sin \theta A^{3}\right)^{2}, \\
g_{7}= & \left(A_{i}-\sigma^{i}\right)^{2}+\frac{1}{2} d \Omega_{4}^{2},
\end{aligned}
$$

$A_{i}$ being the unit charge instantons on $S^{4}$ and $d \Omega_{4}^{2}$ the usual metric for the sphere. 


\subsection{More general quaternion Kahler orbifolds}

The space of metrics defined in (3.28) is very rich. They encode many wellknown examples in the literature as well as new ones. We briefly describe some of them, but a much more complete description can be found in the original references [93, 94].

The Backglund transformation defined in (3.24) is a correspondence between solutions $F$ of (3.29) and solutions of the Ward monopole equation (3.23). The Ward monopole equation describes hyperKahler metrics with two commuting Killing vectors, which in cylindrical co-ordinates take the form [71]

$$
g=\frac{\left(d t+\rho V_{\rho} d \varphi\right)^{2}}{V_{\eta}}+V_{\eta}\left(d \rho^{2}+d \eta^{2}+\rho^{2} d \varphi^{2}\right) .
$$

Several of these metrics were considered recently in [96, 97]. The commuting Killing vectors are $\partial_{t}$ and $\partial_{\varphi}$. It is not difficult to see that these metrics are hyperKahler. By defining the one-form $A=\rho V_{\rho} d \varphi$ and $U=V_{\eta}$, the metrics (3.45) take the Gibbons-Hawking form [113]

$$
g=V^{-1}(d t+A)^{2}+V d x_{i} d x_{j} \delta^{i j},
$$

and it follows that $A$ and $V$ satisfy the linear system of equations

$$
\nabla V=\nabla \times A .
$$

Any element of the family (3.47) is hyperKahler with respect to the hyperKahler triplet

$$
\begin{aligned}
& \bar{J}_{1}=(d t+A) \wedge d x-V d y \wedge d z \\
& \bar{J}_{2}=(d t+A) \wedge d y-V d z \wedge d x \\
& \bar{J}_{3}=(d t+A) \wedge d z-V d x \wedge d y
\end{aligned}
$$

which is actually $t$-independent. Therefore the Killing vector $\partial_{t}$ is triholomorphic. Instead $\partial_{\varphi}$ is not, i.e., it does not preserve (3.48). It follows that the Backglund transformation is a correspondence between toric quaternion Kahler spaces and toric hyperKahler spaces with at least one tri-holomorphic isometry.

The elemental solution of the equation (3.47) is given by the single $\mathrm{Wu}-$ Yang monopole potential $A$ and the scalar field $V$ of the form

$$
V=c+\frac{a}{r}, \quad A=\frac{a(y d x-x d y)}{r(r+z)} \quad z>0, \quad \widetilde{A}^{\prime}=\frac{a(y d x-x d y)}{r(r-z)} \quad z \leq 0,
$$


$r$ being the radius; $r=\sqrt{x^{2}+y^{2}+z^{2}}$. The vector potential $A$ is not globally defined in $\mathbf{R}^{3}$ due to the presence of Dirac string singularities in the upper $z$ axis for $A$ and in the lower $z$ axis for $\widetilde{A}^{\prime}$. In the overlapping region both potentials differ from one another by a gauge transformation $\widetilde{A}^{\prime}=A-2 a d \arctan (y / x)$. Any array of Dirac monopoles will describe an hyperKahler metric, but only if such monopoles are aligned along an axis, then we will have axial symmetry as in the Ward case (3.45). The elementary Ward solutions are of the form

$$
U_{i}=a_{i} \log \left(\eta-\eta_{i}+\sqrt{\left(\eta-\eta_{i}\right)^{2}+\rho^{2}}\right)
$$

and represent a monopole located in the position $\left(0, \eta_{i}\right)$. Any superposition of such elementary functions will give rise to a toric hyperKahler metric. The Backglund transformation of the solutions $U_{i}$ is given by

$$
F_{i}=\frac{\sqrt{a_{i}^{2} \rho^{2}+\left(a_{i} \eta-\eta_{i}\right)^{2}}}{\sqrt{\rho}} .
$$

Any superposition of these solutions, namely

$$
F=\sum_{k=1}^{m} \frac{\sqrt{a_{k}^{2} \rho^{2}+\left(a_{k} \eta-b_{k}\right)^{2}}}{\sqrt{\rho}} .
$$

will give rise to a toric quaternion Kahler metric. There are also elementary solutions $F=\rho^{3 / 2}$ and $F=\rho^{-1 / 2}$, which are $\eta$-independent.

For $m=2$ the solutions are called two-pole functions and are given by

$$
F_{1}=\frac{1+\sqrt{\rho^{2}+\eta^{2}}}{\sqrt{\rho}} ; \quad F_{2}=\frac{\sqrt{(\rho)^{2}+(\eta+1)^{2}}}{\sqrt{\rho}}-\frac{\sqrt{(\rho)^{2}+(\eta-1)^{2}}}{\sqrt{\rho}} .
$$

The first one gives rise to the spherical metric, while the second one gives rise to the hyperbolic metric. This is seen by defining the co-ordinates $\left(r_{1}, r_{2}\right)$ which are related to the hyperbolic ones $(\rho, \eta)$ by the relation

$$
\left(r_{1}+i r_{2}\right)^{2}=\frac{\eta-1+i \rho}{\eta+1+i \rho} .
$$

By writing the corresponding metric in terms of $(\rho, \eta)$ and making the change to $\left(r_{1}, r_{2}\right)$ gives [93]

$$
g=\left(1-r_{1}^{2}-r_{2}^{2}\right)^{-2}\left(d r_{1}^{2}+d r_{2}^{2}+r_{1}^{2} d \theta_{1}^{2}+r_{2}^{2} d \theta_{2}^{2}\right),
$$

which is the hyperbolic metric on the unit ball on $R^{4}$. This is a conformally flat metric.

Now, let us discuss the case of two monopoles on the $z$ axis. Without loosing generality, it can be considered that the monopoles are located in 
the positions $(0,0, \pm c)$. The potentials for this configurations are

$$
\begin{aligned}
V=\frac{1}{r_{+}}+\frac{1}{r_{-}}, & A=A_{+}+A_{-}=\left(\frac{z_{+}}{r_{+}}+\frac{z_{-}}{r_{-}}\right) d \arctan \left(\frac{y}{x}\right), \\
r_{ \pm} & =\sqrt{x^{2}+y^{2}+(z \pm m)^{2}} .
\end{aligned}
$$

This case corresponds to the Eguchi-Hanson instanton, whose metric, in Cartesian co-ordinates, reads

$$
\begin{aligned}
g= & \left(\frac{1}{r_{+}}+\frac{1}{r_{-}}\right)^{-1}\left(d t+\left(\frac{z_{+}}{r_{+}}+\frac{z_{-}}{r_{-}}\right) d \arctan \left(\frac{y}{x}\right)\right)^{2} \\
& +\left(\frac{1}{r_{+}}+\frac{1}{r_{-}}\right)\left(d x^{2}+d y^{2}+d z^{2}\right) .
\end{aligned}
$$

In order to recognize the Eguchi-Hanson metric in its standard form it is convenient to introduce a new parameter $a^{2}=8 m$, and the elliptic coordinates defined by

$$
x=\frac{r^{2}}{8} \sqrt{1-\left(\frac{a}{r}\right)^{4}} \sin \varphi \cos \theta, \quad y=\frac{r^{2}}{8} \sqrt{1-\left(\frac{a}{r}\right)^{4}} \sin \varphi \sin \theta, \quad z=\frac{r^{2}}{8} \cos \varphi .
$$

In this co-ordinate system it can be checked that

$$
\begin{gathered}
r_{ \pm}=\frac{r^{2}}{8}\left(1 \pm\left(\frac{a}{r}\right)^{2} \cos \varphi\right), \quad z_{ \pm}=\frac{r^{2}}{8}\left(\cos \varphi \pm\left(\frac{a}{r}\right)^{2}\right), \\
V=\frac{16}{r^{2}}\left(1-\left(\frac{a}{r}\right)^{4} \cos ^{2} \varphi\right)^{-1}, \\
A=2\left(1-\left(\frac{a}{r}\right)^{4} \cos ^{2} \varphi\right)^{-1}\left(1-\left(\frac{a}{r}\right)^{4}\right) \cos \varphi d \theta,
\end{gathered}
$$

and, with the help of these expressions, it is found

$$
\begin{aligned}
g= & \frac{r^{2}}{4}\left(1-\left(\frac{a}{r}\right)^{4}\right)(d \theta+\cos \varphi d \tau)^{2}+\left(1-\left(\frac{a}{r}\right)^{4}\right)^{-1} d r^{2} \\
& +\frac{r^{2}}{4}\left(d \varphi^{2}+\sin ^{2} \varphi d \tau\right)
\end{aligned}
$$

being $\tau=2 t$. This is actually a more familiar expression for the EguchiHanson instanton, indeed [114]. Let us also note that the Eguchi-Hanson metric corresponds to two monopoles in the $z$ axis, but if we choose $m^{2}=-1$ this will correspond to the potential for an axially symmetric circle of charge. The corresponding metric is called Eguchi-Hanson metric of the type I, and is always incomplete.

Let us go back to the Backglund transformed geometry corresponding to the Eguchi-Hanson metric. The general "three-pole" solutions can be 
written as

$$
F=\frac{a}{\sqrt{\rho}}+\frac{b+c / m}{2} \frac{\sqrt{\rho^{2}+(\eta+m)^{2}}}{\sqrt{\rho}}+\frac{b-c / m}{2} \frac{\sqrt{\rho^{2}+(\eta-m)^{2}}}{\sqrt{\rho}} .
$$

By definition $-m^{2}= \pm 1$, which means that $m$ can be imaginary or real. The corresponding solutions are denominated as type I and type II, respectively, by analogy with the hyperKahler case. It is interesting to note that for $c=0$ and $b=-1$ and defining the co-ordinates $(t, \theta)$ by $\eta=\left(\cosh ^{2} t-1\right) \cos \theta$ and $\rho=2 \operatorname{coth} t \sinh ^{-1} t \sin \theta$ the metric takes the form

$$
g_{q}=4 d t^{2}+\sinh ^{2} t\left(d \theta^{2}+\sin ^{2} \theta d \varphi^{2}\right)+\frac{\sinh ^{2} 2 t}{2}(d \psi+\cos \theta d \varphi)^{2} .
$$

By making further transformation $2 t=\log (1+r)-\log (1-r)$ this metric takes the form (3.41) and therefore is the Bargmann metric. The Bargmann metric can also be obtained with the function $F=\rho^{3 / 2}$ and this means that different solutions of the equation (3.29) can give rise to the same metric. In fact, the Bargmann metric can be recovered for $c=0$ and $b=1$ and also for $c=1$ and $b=0$. There exists certain freedom in the choice of $F$ that leaves the metric invariant. This freedom allows in particular to set $a=1$. If $c=0$ and $b$ is arbitrary then the metric reduces to the Pedersen metric [99] (see also [95])

$$
g_{q}=\frac{\left(w^{2}+q^{2}\right)}{\left(w-s q r^{2}\right)^{2}}\left(\frac{w r^{2}+s}{1+q r^{2}} d r^{2}+\frac{r^{2}}{4}\left(\frac{1+q r^{4}}{w r^{2}+s} \sigma_{1}^{2}+\left(w r^{2}+s\right)\left(\sigma_{2}^{2}+\sigma_{3}^{2}\right)\right)\right),
$$

$w, q$ and $s$ being three parameters. We should be aware that these metrics possesses only one parameter up to an homothety, the other two can be selected to one by a suitable rescaling. This is in accordance with that, by construction, the only parameter is $b$. The advantage of this notation is that several limits are better understood. The scalar curvature of this metrics is $-48 w q /\left(w^{2}+q^{2}\right)$ and we see that in the limit $w=0$ or $q=0$ the metric will be hyperKahler. In the first case the metric reduces to the Taub-Nut one [115], in the second corresponds to the I and II Eguchi-Hanson metrics.

It is natural to introduce the cylindrical co-ordinate system

$$
\rho=\sqrt{R^{2} \pm 1} \cos \theta, \quad \eta=R \sin \theta
$$

where $\theta$ takes values in the interval $(-\pi / 2, \pi / 2)$. In these co-ordinates

$$
\begin{aligned}
\sqrt{\rho} F & =1+b R+c \sin \theta \\
\rho^{-1}\left[\frac{1}{4} F^{2}-\rho^{2}\left(F_{\rho}^{2}+F_{\eta}^{2}\right)\right] & =\frac{b(R \mp b)+c(\sin \theta+c)}{R^{2} \pm \sin ^{2} \theta} .
\end{aligned}
$$

The zeroes of $F$ are the conformal infinite of the metric, while the zeroes of $\frac{1}{4} F^{2}-\rho^{2}\left(F_{\rho}^{2}+F_{\eta}^{2}\right)$ are singularities separating the domains of positive and 
negative scalar curvatures. For the type II metrics the co-ordinate $R$ can take values between 1 and $\infty$. For $b=1$ and $c=0$ the Fubbini-Study metric on $\mathrm{CP}(2)$ is obtained, while for $b=-1$ and $c=0$ or $b=0$ and $c= \pm 1$ the Bargmann metric is obtained. By introducing the vector $(b, c)$ we have that along the lines joining these four points the metrics will be bi-axial Bianchi. Along the lines joining $(1,0)$ with the other points there will live Bianchi IX metrics whereas on the lines between $(-1,0),(0,1)$ and $(0,-1)$ the metric is Bianchi VIII. For the type I metrics the value of $R$ is non-restricted. The zeroes of $F$ defining the conformal infinite are $R_{\infty}=-\frac{(1+c \sin \theta)}{b}$. The zeroes of $\frac{1}{4} F^{2}-\rho^{2}\left(F_{\rho}^{2}+F_{\eta}^{2}\right)$ are $R_{ \pm}=\left(b^{2}+c^{2}+c \sin \theta\right) / b$. The case $b=0$ corresponds to Bianchi VIII metrics. If $c=0$ we obtain the Pedersen metrics. The conformal infinite is $R_{\infty}=1 / b$ and $R_{ \pm}=b$ [93].

There have been found certain quaternion Kahler deformations of the Taub-Nut gravitational instantons and other examples in [100], and the relation between these metrics and the $m$-pole solutions has been worked out in that reference. Also we would like to remark that the orbifolds (3.1) can be represented in the form (3.28) but the co-ordinate change is rather complicated and we will not describe it here, see [73, 74]. Higher multiinstanton solutions, or $m$-pole solutions are a linear combinations of the form

$$
F=\sum_{k}^{m} \frac{\sqrt{a_{k}^{2} \rho^{2}+\left(a_{k} \eta-b_{k}\right)^{2}}}{\sqrt{\rho}}
$$

for some real parameters $\left(a_{k}, b_{k}\right)$ for $1<k<m$. But there is an $S L(2, R)$ action that leaves the metric invariant up to an overall factor and therefore, as a vector space, $\left(a_{k}, b_{k}\right)$ is $2 m-\operatorname{dim} S L(2, R)-1=2(m-2)$-dimensional. The $m$-pole solutions arise as quaternion-Kahler quotients of $H P^{m-1}$ by an $(m-2)$-dimensional subtorus of a maximal torus $T^{m}$ in $S p(m)$, all these metrics are therefore defined on a compact orbifolds [77]. Applications of these to the universal hypermultiplet have been found, for instance in [103-105]. Other applications have been considered in [88-90].

\section{Explicit tri-Sasaki and weak $G_{2}$ metrics and supergravity solutions}

\subsection{Tri-Sasaki and weak $G_{2}$ over AdS-Kerr and AdS-Taub-Nut}

We are now in a position to construct compact tri-Sasaki and weak $G_{2}$ holonomy metrics. The main ingredient in this construction is Proposition 2.1, 
applied to limiting cases of the euclidean Plebanski-Demianski solution (3.8) or to the toric metrics (3.28). But before we start it is convenient to mention that there exists in the literature Einstein-Sasaki spaces fibred over the so-called "orthotoric" Kahler-Einstein spaces [40, 41]. As was shown in [41] this space can be obtained by taking certain scaling limit of the Euclidean Plebanski-Demianski metrics (3.8). In particular, there were found several toric Einstein-Sasaki metrics defined over $S^{2} \times S^{3}$. Nevertheless, those spaces are five-dimensional and are fibrations over four-dimensional KahlerEinstein spaces. Instead, we are presenting a seven-dimensional family which is fibred over four-dimensional quaternion Kahler spaces. Thus, in principle, our examples bear no relation to those found in $[40,41]$.

After this comment, we turn our attention now to the construction of tri-Sasaki (and weak $G_{2}$ ) metrics implicit in Proposition 2.1.

The AdS-Taub-Nut case. It is direct, by using Proposition 2.1 and the lifting formula (2.34), to work out tri-Sasaki and weak $G_{2}$ holonomy metrics fibred over the AdS-Taub-Nut metrics (3.5), the result is

$$
\begin{aligned}
g_{7}= & \left(\sqrt{(\widetilde{r}+N) V(\widetilde{r})} \sin \tilde{\theta} d \widetilde{\phi}+\sigma_{1}\right)^{2}+\left((\widetilde{r}-N) d \widetilde{t}+g(\widetilde{r}) \cos \widetilde{\theta} d \widetilde{\phi}-\sigma_{2}\right)^{2} \\
& +\left(\sqrt{(\widetilde{r}+N) V(\widetilde{r})} d \widetilde{\theta}-\sigma_{3}\right)^{2}+b\left(V(\widetilde{r})(d \widetilde{t}-2 N \cos \widetilde{\theta} d \widetilde{\phi})^{2}\right. \\
& \left.+\frac{d \widetilde{r}^{2}}{V(\widetilde{r})}+\left(\widetilde{r}^{2}-N^{2}\right)\left(d \widetilde{\theta}^{2}+\sin ^{2} \widetilde{\theta} d \widetilde{\phi}^{2}\right)\right)
\end{aligned}
$$

Although the base quaternion Kahler space possess $S U(2) \times U(1)$ isometry, this group does not preserve the fibres, so the isometry group is $S U(2)^{\prime} \times$ $U(1)^{2}$, the $S U(2)^{\prime}$ group being related to the Maurer-Cartan forms of the fibre metric and $U(1)^{2}$ generated by $\partial_{\tilde{t}}$ and $\partial_{\widetilde{\phi}}$. Let us notice that we have a third commuting Killing vector, which is the Reeb vector $\partial_{\tau}$, which is present in the expression for the Maurer-Cartan forms $\sigma_{i}$. Therefore we have a $T^{3}$ subgroup of isometries. By taking into account the explicit form of the $\sigma_{i}$ s given in (2.4) we obtain the following metric components

$$
\begin{aligned}
& g_{\widetilde{t t}}=(\widetilde{r}-N)^{2}+b V(\widetilde{r}), \quad g_{\widetilde{\phi} \widetilde{\phi}}=4 b N^{2} V(\widetilde{r}) \cos ^{2} \widetilde{\theta}+\left(\widetilde{r}^{2}-N^{2}\right) \sin ^{2} \widetilde{\theta} \\
& g_{\widetilde{\theta \theta}}=b\left(\widetilde{r}^{2}-N^{2}\right)+(\widetilde{r}+N) V(\widetilde{r}), \quad g_{\widetilde{r} \widetilde{r}}=\frac{b}{V(\widetilde{r})}, \quad g_{\tau \tau}=g_{\varphi \varphi}=g_{\theta \theta}=1 \\
& g_{\widetilde{t \phi}}=-2 N b V(\widetilde{r}) \cos \widetilde{\theta}+(\widetilde{r}-N) g(\widetilde{r}) \cos \widetilde{\theta}, \quad g_{\widetilde{t} \tau}=-(\widetilde{r}-N) \cos \varphi \sin \theta \\
& g_{\widetilde{\phi} \tau}=\sqrt{(\widetilde{r}+N) V(\widetilde{r})} \sin \widetilde{\theta} \sin \theta \sin \varphi+g(\widetilde{r}) \cos \widetilde{\theta} \sin \theta \cos \varphi \\
& g_{\widetilde{\phi} \theta}=\sqrt{(\widetilde{r}+N) V(\widetilde{r})} \sin \widetilde{\theta} \cos \varphi+g(\widetilde{r}) \cos \widetilde{\theta} \sin \varphi
\end{aligned}
$$




$$
\begin{aligned}
g_{\widetilde{\theta} \tau} & =-\sqrt{(\widetilde{r}+N) V(\widetilde{r})} \cos \theta, \quad g_{\widetilde{\theta} \varphi}=-\sqrt{(\widetilde{r}+N) V(\widetilde{r})} \\
g_{\tilde{t} \theta} & =-(\widetilde{r}-N) \sin \varphi \sin \theta, \quad g_{\tau \varphi}=\cos \theta,
\end{aligned}
$$

the remaining components are all zero. The parameter $b$ takes the values 1 or $5, b=1$ corresponds to an Einstein-Sasaki metric, while $b=5$ corresponds to a weak $G_{2}$ holonomy metric.

The AdS-Kerr-Newman case. For the rotating case, i.e., for the AdSKerr-Newman metrics (3.8) we obtain the metrics

$$
\begin{aligned}
g_{q}= & \left(\frac{\sqrt{f(\widetilde{\theta}) c(\widetilde{r}) d(\widetilde{r})}}{e(\widetilde{r}, \widetilde{\theta})} \frac{\sin \widetilde{\theta}}{\Xi} d \widetilde{\phi}-\sigma_{1}\right)^{2}+\left(e(\widetilde{r}, \widetilde{\theta}) d \widetilde{t}+\frac{W(\widetilde{r}, \widetilde{\theta})}{\Xi e(\widetilde{r}, \widetilde{\theta})} d \widetilde{\phi}-\sigma_{2}\right)^{2} \\
& +\left(\sqrt{\frac{c(\widetilde{r}) d(\widetilde{r})}{f(\widetilde{\theta})}} \frac{d \widetilde{\theta}}{e(\widetilde{r}, \widetilde{\theta})}-\sqrt{\frac{f(\widetilde{\theta})}{c(\widetilde{r}) d(\widetilde{r})}} \frac{a \sin \widetilde{\theta}}{e(\widetilde{r}, \widetilde{\theta})} d \widetilde{r}-\sigma_{3}\right)^{2} \\
& +\frac{b f(\widetilde{\theta}) \sin ^{2} \widetilde{\theta}}{\widetilde{r}^{2}-a^{2} \cos ^{2} \widetilde{\theta}}\left(a d \widetilde{t}-\frac{c(\widetilde{r})}{\Xi} d \widetilde{\phi}\right)^{2}+\frac{b c(\widetilde{r}) d(\widetilde{r})}{\widetilde{r}^{2}-a^{2} \cos \widetilde{\theta}^{2}}\left(d \widetilde{t}+\frac{a \sin ^{2} \widetilde{\theta}}{\Xi} d \widetilde{\phi}\right)^{2} \\
& +\frac{\widetilde{r}^{2}-a^{2} \cos \widetilde{\theta}^{2}}{f(\widetilde{\theta})} b d \widetilde{\theta}^{2}+\frac{\widetilde{r}^{2}-a^{2} \cos \widetilde{\theta}^{2}}{c(\widetilde{r}) d(\widetilde{r})} b d \widetilde{r}^{2},
\end{aligned}
$$

where we have introduced the functions

$$
\begin{aligned}
f(\widetilde{\theta}) & =1-a^{2} \cos ^{2} \widetilde{\theta}, \quad c(\widetilde{r})=\widetilde{r}^{2}-a^{2}, \\
d(\widetilde{r}) & =1-\widetilde{r}^{2}, \quad e(\widetilde{r}, \widetilde{\theta})=\widetilde{r}-a \cos \widetilde{\theta} .
\end{aligned}
$$

The local isometry is $S U(2) \times U(1)^{2}$ and as before, the vectors $\partial_{\tilde{t}}, \partial_{\widetilde{\phi}}$ and $\partial_{\tau}$ generate a $T^{3}$ isometry subgroup. From expression (4.2) we read the following components

$$
\begin{aligned}
g_{\widetilde{t t}}= & \frac{b c(\widetilde{r}) d(\widetilde{r})}{\widetilde{r}^{2}-a^{2} \cos \widetilde{\theta}^{2}}+a^{2} \frac{b f(\widetilde{\theta}) \sin ^{2} \widetilde{\theta}}{\widetilde{r}^{2}-a^{2} \cos ^{2} \widetilde{\theta}}+e^{2}(\widetilde{r}, \widetilde{\theta}) \\
g_{\widetilde{\phi} \widetilde{\phi}}= & \frac{b c(\widetilde{r}) d(\widetilde{r})}{\widetilde{r}^{2}-a^{2} \cos \widetilde{\theta}^{2}} \frac{a^{2} \sin ^{4} \widetilde{\theta}}{\Xi^{2}}+\frac{b f(\widetilde{\theta}) \sin ^{2} \widetilde{\theta}}{\widetilde{r}^{2}-a^{2} \cos ^{2} \widetilde{\theta}} \frac{c^{2}(\widetilde{r})}{\Xi^{2}}+\frac{W^{2}(\widetilde{r}, \widetilde{\theta})}{\Xi^{2} e^{2}(\widetilde{r}, \widetilde{\theta})} \\
& +\frac{f(\widetilde{\theta}) c(\widetilde{r}) d(\widetilde{r})}{e^{2}(\widetilde{r}, \widetilde{\theta})} \frac{\sin ^{2} \widetilde{\theta}}{\Xi^{2}}
\end{aligned}
$$




$$
\begin{aligned}
& g_{\widetilde{\theta} \widetilde{\theta}}=\frac{\widetilde{r}^{2}-a^{2} \cos \widetilde{\theta}^{2}}{f(\widetilde{\theta})} b+\frac{1}{e^{2}(\widetilde{r}, \widetilde{\theta})} \frac{c(\widetilde{r}) d(\widetilde{r})}{f(\widetilde{\theta})}, \\
& g_{\widetilde{r} \widetilde{r}}=\frac{\widetilde{r}^{2}-a^{2} \cos \widetilde{\theta}^{2}}{c(\widetilde{r}) d(\widetilde{r})} b+\frac{f(\widetilde{\theta})}{c(\widetilde{r}) d(\widetilde{r})} \frac{a^{2} \sin ^{2} \widetilde{\theta}}{e^{2}(\widetilde{r}, \widetilde{\theta})}, \\
& g_{\widetilde{r} \widetilde{\theta}}=-\frac{a \sin \widetilde{\theta}}{e^{2}(\widetilde{r}, \widetilde{\theta})}, \quad g_{\widetilde{r} \varphi}=\sqrt{\frac{f(\widetilde{\theta})}{c(\widetilde{r}) d(\widetilde{r})}} \frac{a \sin \widetilde{\theta}}{e(\widetilde{r}, \widetilde{\theta})}, \\
& g_{\widetilde{r} \tau}=\sqrt{\frac{f(\widetilde{\theta}) \cos \theta}{c(\widetilde{r}) d(\widetilde{r})}} \frac{a \sin \widetilde{\theta} \cos \theta}{e(\widetilde{r}, \widetilde{\theta})} \\
& g_{\widetilde{t} \widetilde{\phi}}=\frac{b c(\widetilde{r}) d(\widetilde{r})}{\widetilde{r}^{2}-a^{2} \cos \widetilde{\theta}^{2}} \frac{a \sin ^{2} \widetilde{\theta}}{\Xi}+a \frac{b f(\widetilde{\theta}) \sin ^{2} \widetilde{\theta}}{\widetilde{r}^{2}-a^{2} \cos ^{2} \widetilde{\theta}} \frac{c(\widetilde{r})}{\Xi}+\frac{W(\widetilde{r}, \widetilde{\theta})}{\Xi}, \\
& g_{\tau \tau}=g_{\varphi \varphi}=g_{\theta \theta}=1, \quad g_{\widetilde{t} \tau}=-e(\widetilde{r}, \widetilde{\theta}) \cos \varphi \sin \theta \\
& g_{\widetilde{\phi} \tau}=-\frac{\sqrt{f(\widetilde{\theta}) c(\widetilde{r}) d(\widetilde{r})}}{e(\widetilde{r}, \widetilde{\theta})} \frac{\sin \widetilde{\theta}}{\Xi} \sin \theta \sin \varphi-\frac{W(\widetilde{r}, \widetilde{\theta})}{\Xi e(\widetilde{r}, \widetilde{\theta})} \sin \theta \cos \varphi \\
& g_{\widetilde{\phi} \theta}=\frac{W(\widetilde{r}, \widetilde{\theta})}{\Xi e(\widetilde{r}, \widetilde{\theta})} \sin \varphi+\frac{\sqrt{f(\widetilde{\theta}) c(\widetilde{r}) d(\widetilde{r})}}{e(\widetilde{r}, \widetilde{\theta})} \frac{\sin \widetilde{\theta}}{\Xi} \cos \varphi \\
& g_{\widetilde{\theta} \tau}=-\sqrt{\frac{c(\widetilde{r}) d(\widetilde{r})}{f(\widetilde{\theta})}} \frac{\cos \theta}{e(\widetilde{r}, \widetilde{\theta})}, \quad g_{\widetilde{\theta} \varphi}=-\sqrt{\frac{c(\widetilde{r}) d(\widetilde{r})}{f(\widetilde{\theta})}} \frac{1}{e(\widetilde{r}, \widetilde{\theta})} \\
& g_{\widetilde{t} \theta}=-e(\widetilde{r}, \widetilde{\theta}) \sin \varphi, \quad g_{\tau \varphi}=\cos \theta
\end{aligned}
$$

and the other components are zero. In the limit $a=0$ the base metric reduces to $S^{4}$, the resulting tri-Sasaki metrics is

$$
\begin{aligned}
g_{7}= & (\sin \widetilde{\rho} d \widetilde{t}+\cos \widetilde{\theta} d \widetilde{\phi}+\sin \varphi d \theta-\cos \varphi \sin \theta d \tau)^{2}+(\cos \widetilde{\rho} d \widetilde{\theta}-d \varphi-\cos \theta d \tau)^{2} \\
& +(\cos \widetilde{\rho} \sin \widetilde{\theta} d \widetilde{\phi}-\cos \varphi d \theta-\sin \varphi \sin \theta d \tau)^{2}+b \cos ^{2} \widetilde{\rho} d \widetilde{t}^{2}+b d \widetilde{\rho}^{2} \\
& +b \sin ^{2} \widetilde{\rho}\left(d \widetilde{\theta}^{2}+\sin ^{2} \widetilde{\theta} d \widetilde{\phi}^{2}\right) .
\end{aligned}
$$

Finally we should recall that the tri-Sasaki and weak $G_{2}$ metrics fibred over $C P(2)$ are those corresponding to the spaces $N(1,1)_{I}$ and $N(1,1)_{I I}$ and were given in (2.37), so we will not discuss them again.

\subsection{An infinite family of weak G2 and tri-Sasaki orbifolds}

Let us now turn our attention to the construction of the tri-Sasaki and the Kahler-Einstein metrics fibred over the toric quaternion Kahler metrics (3.29). The anti-self-dual part of the spin connection $\omega-^{i}$ possesses a 
remarkable simple form in terms of the potential function $F$ [93]

$$
\omega_{-}^{1}=\frac{1}{F}\left[-\rho F_{\eta} \frac{d \rho}{\rho}+\left(\frac{1}{2} F+\rho F_{\rho}\right) \frac{d \eta}{\rho}\right], \quad \omega_{-}^{2}=\frac{u}{F}, \quad \omega_{-}^{3}=\frac{v}{F} .
$$

The Kahler-Einstein metric defined over the twistor space $Z$ of (3.28) is obtained directly from (2.22) and is given by

$$
\begin{aligned}
g_{6}= & \frac{F^{2}-4 \rho^{2}\left(F_{\rho}^{2}+F_{\eta}^{2}\right)}{4 F^{2}} \frac{d \rho^{2}+d \eta^{2}}{\rho^{2}} \\
& +\frac{\left[\left(F-2 \alpha F_{\rho}\right) u-2 \rho F_{\eta} v\right]^{2}+\left[-2 \rho F_{\eta} u+\left(F+2 \rho F_{\rho}\right) v\right]^{2}}{F^{2}\left[F^{2}-4 \rho^{2}\left(F_{\rho}^{2}+F_{\eta}^{2}\right)\right]} \\
& +\left(d \theta-\sin \varphi \omega_{-}^{2}+\cos \varphi \omega_{-}^{1}\right)^{2}+\left(\sin \theta d \varphi-\cos \theta \sin \varphi \omega_{-}^{1}\right. \\
& \left.-\cos \theta \cos \varphi \omega_{-}^{2}+\sin \theta \omega_{-}^{3}\right)^{2} .
\end{aligned}
$$

The Kahler form for (4.6) is $\bar{J}=d H$ where

$$
\begin{aligned}
H= & \frac{\sin \theta \sin \varphi}{2 \rho F}\left(-2 \rho F_{\eta} d \rho+\left(F+2 \rho F_{\rho}\right) d \eta\right)+\frac{\sin \theta \cos \varphi u}{F} \\
& +\frac{\cos \theta v}{F}-\cos \theta d \varphi,
\end{aligned}
$$

and with the help of this expression we obtain the tri-Sasaki metrics

$$
g_{7}=(d \tau+H)^{2}+g_{6},
$$

$H$ being defined in (4.7). Both expressions for the tri-Sasaki and the KahlerEinstein metrics are completely determined in terms of a single eigenfunction $F$ of the hyperbolic Laplacian. Indeed the components of $g_{7}$ are given explicitly by

$$
\begin{aligned}
\left(g_{7}\right)_{\rho \rho}= & \left(g_{q}\right)_{\rho \rho}+\frac{F_{\eta}^{2}}{F^{2}}, \quad\left(g_{7}\right)_{\rho \eta}=\frac{F_{\eta}}{F^{2}}\left(F+2 \rho F_{\rho}\right), \quad\left(g_{7}\right)_{\eta \eta}=\left(g_{q}\right)_{\eta \eta} \\
& +\frac{1}{\rho^{2} F^{2}}\left(\frac{F}{2}+\rho F_{\rho}\right)^{2}, \\
\left(g_{7}\right)_{\alpha \alpha}= & \left(g_{q}\right)_{\alpha \alpha}+\frac{\left(1+\rho^{2}\right)}{\rho F^{2}}, \quad\left(g_{7}\right)_{\alpha \beta}=\left(g_{q}\right)_{\alpha \beta} \\
& +\frac{2 \eta}{\rho F^{2}}, \quad\left(g_{7}\right)_{\beta \beta}=\left(g_{q}\right)_{\beta \beta}+\frac{1}{\rho F^{2}} \\
\left(g_{7}\right)_{\rho \theta}= & \frac{2 F_{\eta}}{F} \cos \theta, \quad\left(g_{7}\right)_{\rho \tau}=\frac{2 F_{\eta}}{F} \sin \theta \sin \varphi
\end{aligned}
$$




$$
\begin{aligned}
& \left(g_{7}\right)_{\eta \theta}=-\frac{1}{\rho F}\left(F+2 \rho F_{\rho}\right) \cos \theta, \quad\left(g_{7}\right)_{\eta \tau}=-\frac{1}{\rho F}\left(F+2 \rho F_{\rho}\right) \sin \theta \sin \varphi \\
& \left(g_{7}\right)_{\alpha \theta}=\frac{2 \sqrt{\rho}}{F} \sin \varphi, \quad\left(g_{7}\right)_{\alpha \varphi}=-\frac{2 \eta}{\sqrt{\rho} F}, \\
& \left(g_{7}\right)_{\alpha \tau}=-\frac{2 \sqrt{\rho}}{F} \sin \theta \cos \varphi+\frac{2 \eta}{\sqrt{\rho} F} \cos \theta, \\
& \left(g_{7}\right)_{\beta \varphi}=-\frac{2}{\sqrt{\rho} F}, \quad\left(g_{7}\right)_{\beta \tau}=-\frac{2}{\sqrt{\rho} F} \cos \theta \\
& \left(g_{7}\right)_{\theta \theta}=\left(g_{7}\right)_{\tau \tau}=\left(g_{7}\right)_{\varphi \varphi}=1, \quad\left(g_{7}\right)_{\tau \varphi}=\cos \theta
\end{aligned}
$$

and the remaining components are zero.

The tri-Sasaki metric possesses an $S U(2)$ isometry group associated with the $\sigma^{i}$ and a $T^{2}$ isometry of the quaternion Kahler base. Therefore the isometry group is at least $S O(3) \times T^{2}$. The Killing vectors are

$$
\begin{aligned}
& K_{1}=\partial_{\alpha}, \quad K_{2}=\partial_{\beta}, \quad K_{3}=\partial_{\tau} \\
& K_{4}=\cos \tau \partial_{\varphi}-\operatorname{coth} \varphi \sin \tau \partial_{\tau}+\frac{\sin \tau}{\sin \varphi} \partial_{\theta} \\
& K_{5}=-\sin \tau \partial_{\varphi}-\operatorname{coth} \varphi \cos \tau \partial_{\tau}+\frac{\cos \tau}{\sin \varphi} \partial_{\theta}
\end{aligned}
$$

with commutation rule

$$
\begin{aligned}
& {\left[K_{1}, K_{i}\right]=\left[K_{2}, K_{i}\right]=0, \quad i=1, \ldots, 5} \\
& {\left[K_{i}, K_{j}\right]=\epsilon_{i j k} K_{k}, \quad i, j, k=3,4,5 .}
\end{aligned}
$$

Both the tri-Sasaki metric and the Kahler-Einstein possess three commuting Killing vectors. For the Kahler-Einstein metric the vectors are $\partial_{\theta}, \partial_{\alpha}$ and $\partial_{\beta}$, for the tri-Sasaki metric they are $\partial_{\tau}, \partial_{\alpha}$ and $\partial_{\beta}$.

By making the replacement $g_{q} \rightarrow 5 g_{q}$ in the formulae before we obtain a family of weak $G_{2}$ holonomy metric. Locally the isometry group will be the same for them than for the tri-Sasaki ones that we have presented.

\subsection{Supergravity solutions fibred over Einstein spaces}

Let us describe how to construct supergravity backgrounds fibred over conical Ricci-flat metrics and their role in the AdS/CFT correspondence. 
Consider an stack of $N$ parallel Dp branes. The general form of such background is

$$
\begin{aligned}
g_{10} & =H_{p}^{-1 / 2}(r) g_{1, p}+H_{p}^{1 / 2}(r)\left(d r^{2}+r^{2} g_{8-p}\right), \\
e^{2 \phi-2 \phi_{\infty}} & =H_{p}^{(p-3) / 2}, \quad A_{p+1}=-\frac{1}{2}\left(H_{p}^{-1}-1\right) d x^{0} \wedge \ldots \wedge d x^{p},
\end{aligned}
$$

where

$$
H_{p}(r)=1+2^{5-p} \pi^{(5-p) / 2} g_{s} N_{c} \Gamma\left(\frac{7-p}{2}\right) \frac{\alpha^{\prime(7-p) / 2}}{r^{7-p}} .
$$

The metric $g_{8-p}$ is Einstein and is assumed to be independent on $r$ and also independent on the Minkowski co-ordinates $(t, x, y, z)$. If the Dp branes are flat, the light open spectrum is $U\left(N_{c}\right)$ super Yang-Mills in $p+1$ dimensions. We have that $g_{Y M}=2 \pi^{p-2} g_{s} \alpha^{\prime(p-3) / 2}$ being $g_{s}=e^{2 \phi_{\infty}}$. The field theory limit is obtained by taking $\alpha \rightarrow 0$ such that $g_{Y M}$ is fixed. For $p<3$ the ten-dimensional Newton constant goes to zero and the theory is decoupled from the bulk. Instead for $p>3$ the constant $g_{s}$ goes to infinite and a dual description is convenient in order to analyse the decoupling problem. In order to have finite energy configurations in the field theory limit one should consider the near horizon limit in the IIB background. Such limit is obtained by taking $r \rightarrow 0$ and $\alpha^{\prime} \rightarrow 0$ such that the quantity with energy units $U=r / \alpha^{\prime}$ is fixed. For any $p$ the resulting metric will be

$$
\begin{aligned}
g_{I I B} & =\alpha^{\prime}\left[\left(\frac{d_{p} g_{Y M}^{2} N}{U^{7-p}}\right)^{-1 / 2} g_{1, p}+\left(\frac{d_{p} g_{Y M}^{2} N}{U^{7-p}}\right)^{1 / 2}\left(d U^{2}+U^{2} d \Omega_{8-p}\right)\right] \\
e^{\phi} & =(2 \pi)^{2-p} g_{Y M}^{2}\left(\frac{d_{p} g_{Y M}^{2} N}{U^{7-p}}\right)^{(3-p) / 4}, \quad d_{p}=2^{7-2 p} \pi^{(9-3 p) / 2} \Gamma\left(\frac{7-p}{2}\right) .
\end{aligned}
$$

The Yang-Mills coupling constant $g_{Y M}$ is not dimensionless for any $p$, but the effective constant $g_{\text {eff }}^{2} \sim g_{Y M}^{2} N U^{p-3}$ is. The low energy description of super Yang-Mills can be trusted for $g_{\text {eff }}^{2} \ll 1$ which means that $U \gg\left(g_{Y M}^{2} N\right)^{1 /(3-p)}$ for $p<3$ and $U \ll\left(g_{Y M}^{2} N\right)^{1 /(3-p)}$ for $p>3$. In the ultraviolet limit $U \rightarrow \infty$ the string coupling vanish for $p<3$ and the theory is UV free. In the other case a dual description is needed, which is in accordance with the fact that the corresponding SYM theories are not renormalizable and new degrees of freedom appears at short distances. 
The situation is different for $p=3$, in which the AdS/CFT correspondence fully applies. The type IIB supergravity solutions of the form

$$
\begin{aligned}
& g_{10}=H^{-1 / 2}\left(-d t^{2}+d x^{2}+d y^{2}+d z^{2}\right)+H^{1 / 2}\left(d r^{2}+r^{2} g_{5}\right), \\
& e^{2 \phi}=e^{2 \phi_{\infty}}, \quad F_{5}=(1+*) d H_{3}^{-1} \wedge d x^{0} \wedge d x^{1} \wedge d x^{2} \wedge d x^{3},
\end{aligned}
$$

$H(r)$ being a harmonic function over the Ricci flat metric given by

$$
H(r)=1+\frac{L^{4}}{r^{4}}, \quad L^{4}=4 \pi g_{8} N \alpha^{2} .
$$

Such solutions represent an stack of $N$ parallel D3 branes separated by some distance called $r$. Then it follows that the six-dimensional metric in (4.11) possesses a conical singularity, except for the round five sphere. It is also Ricci flat by construction and therefore $g_{5}$ is Einstein. If the Ricci flat metric is indeed flat then the theory living in the D3 brane decouples from the bulk and the branes come close together. The resulting theory is $N=4$ super Yang-Mills. Also the near horizon limit of the geometry of (4.11) is $A d S_{5} \times X_{5}, X_{5}$ being the Einstein space over which $g_{5}$ is defined. In the context of the AdS/CFT correspondence the gauge field theory living on the D3 brane at the conical singularity is identified as the dual of type IIB string theory on $A d S_{5} \times X_{5}$. The open and closed string massive modes decouple by taking the limit $\alpha^{\prime} \rightarrow 0$ and the Planck length $l_{p}=g_{s}^{1 / 2} \alpha^{\prime}$ goes to zero because $g_{s}$ is given in terms of the dilaton, which is constant. The $A d S$ factor reflects that the dual-field theory is conformally invariant. The number of supersymmetries of the gauge theory is related to the number of independent Killing vectors, which depends on the holonomy of the cone. Instead for $p$ is different from 3 the curvature in the near horizon limit is $R \sim \frac{1}{g_{e f f}}$ which is $U$ dependent, thus no AdS factor appears. This reflects that $U\left(N_{c}\right)$ super Yang Mills theory is not conformal invariant. The same happens for non-flat branes.

There are also of interest 11-dimensional supergravity solutions over a manifold with local form $M_{3} \times X_{8}$, the manifold $X_{8}$ being Ricci flat and developing a conical singularity. The generic supergravity solution in consideration is of the form

$$
\begin{aligned}
g_{11} & =H^{-2 / 3}\left(-d t^{2}+d x^{2}+d y^{2}\right)+H^{1 / 3}\left(d r^{2}+r^{2} g_{7}\right), \\
F & = \pm d x \wedge d y \wedge d t \wedge d H^{-1}
\end{aligned}
$$

where

$$
H(r)=1+\frac{2^{5} \pi^{2} N l_{p}^{6}}{r^{6}} .
$$

This solution describes $N$ M2 branes. The near horizon limit of this geometry is obtained taking the 11-dimensional Planck length $l_{p} \rightarrow 0$ and keeping fixed $U=\frac{r^{2}}{l_{p}^{3}}$. The resulting background is $A d S_{4} \times X_{7}, X_{7}$ being an 
Einstein manifold with cosmological constant $\Lambda=5$, and the radius of $A d S_{4}$ is $2 R_{A d S}=l_{p}\left(2^{5} \pi^{2} N\right)^{1 / 6}$. Such solutions have the generic form

$$
g_{11}=g_{A d S}+g_{7}, \quad F_{4} \sim \omega_{A d S},
$$

$\omega_{A d S}$ being the volume form of $A d S_{4}$. If $X_{7}$ is the round sphere the radius will be the same than the $A d S$ part. This is the flat case and it is conjectured that the dual theory is the $2+1$ dimensional $N=8$ superconformal field theory living on the $M 2$ brane. The isometry group $S O(7)$ of the sphere reflects the fact that a $N=8 \mathrm{SCFT}$ is invariant under $S O(7)$ subgroup instead of $S O(8)$. The quantization of the flux of the tensor $F$ implies that the constant $\alpha$ is quantized in units of $l_{11}^{6}, l_{11}$ being the Planck length in 11-dimensions. The backgrounds are in general associated to three-dimensional conformal field theories arising as the infrared limit of the world volume theory of $N$ coincident M2 branes located at the singularity of $M_{3} \times X_{8}$. Also in this case, the number of supersymmetries of the field theory is determined by the holonomy of $X_{8}$. In the case of $\operatorname{Spin}(7), S U(4)$ or $S p(2)$ holonomies we have $N=1,2,3$ supersymmetries, respectively. This implies that the sevendimensional cone will be of weak $G_{2}$ holonomy (if the eight-dimensional metric is of cohomogenity one, see next), tri-Sasaki or a Sasaki-Einstein, respectively. If $g_{8}$ is flat, then we have the maximal number of supersymmetries, namely eight.

\subsection{Supergravity backgrounds over tri-Sasaki and weak $G_{2}$}

Let us construct supergravity backgrounds corresponding to the Einstein seven-metrics (4.8) or (4.1), (4.3) and (4.4). The generic 11-dimensional supergravity solution is

$$
\begin{aligned}
g_{11}= & H^{-2 / 3}\left(-d t^{2}+d x^{2}+d y^{2}\right) \\
& +H^{1 / 3} d r^{2}+r^{2} H^{1 / 3}\left(\left(g_{7}\right)_{\alpha \alpha} d \alpha^{2}+\left(g_{7}\right)_{\alpha \beta} d \alpha \otimes d \beta\right. \\
& +\left(g_{7}\right)_{\alpha \phi} d \alpha \otimes d \phi+\left(g_{7}\right)_{\beta \beta} d \beta^{2}+\left(g_{7}\right)_{\beta \phi} d \beta \otimes d \phi+\left(g_{7}\right)_{\phi \phi} d \phi^{2} \\
& \left.+Q_{\alpha} d \alpha+Q_{\beta} d \beta+Q_{\phi} d \phi+\widetilde{H}\right), \\
F= & \pm d x \wedge d y \wedge d t \wedge d H^{-1}
\end{aligned}
$$

$H$ being a harmonic function over the hyperKahler cone. In particular if $H=H(r)$ we have

$$
H(r)=1+\frac{2^{5} \pi^{2} N l_{p}^{6}}{r^{6}}
$$


In the expression for the metric we have introduced the one-forms $Q$ and the symmetric tensor $\widetilde{H}$ given by

$$
\begin{aligned}
\widetilde{H}= & d \theta^{2}+d \varphi^{2}+\left(g_{7}\right)_{\theta \eta} d \theta \otimes d \eta+\left(g_{7}\right)_{\rho \rho} d \rho^{2}+\left(g_{7}\right)_{\rho \eta} d \rho \otimes d \eta+\left(g_{7}\right)_{\eta \eta} d \eta^{2}, \\
Q_{\alpha}= & \left(g_{7}\right)_{\alpha \theta} d \theta+\left(g_{7}\right)_{\alpha \varphi} d \varphi, \quad Q_{\beta}=\left(g_{7}\right)_{\beta \varphi} d \varphi, \quad Q_{\phi}=\left(g_{7}\right)_{\phi \varphi} d \varphi \\
& +\left(g_{7}\right)_{\phi \eta} d \eta+\left(g_{7}\right)_{\phi \rho} d \rho .
\end{aligned}
$$

This supergravity solution describes $N$ M2 branes. The near horizon limit of this geometry is obtained by taking the 11-dimensional Planck length $l_{p} \rightarrow 0$ and keeping fixed $U=r^{2} / l_{p}^{3}$. The resulting background is $A d S_{4} \times X_{7}, X_{7}$ being an Einstein manifold with cosmological constant $\Lambda=5$, and the radius of $A d S_{4}$ is $2 R_{A d S}=l_{p}\left(2^{5} \pi^{2} N\right)^{1 / 6}$. Such solutions have the generic form

$$
g_{11}=g_{A d S}+g_{7}, \quad F_{4} \sim \omega_{A d S},
$$

$g_{7}$ being an Einstein metric over $X_{7}$ and $\omega_{A d S}$ the volume form of $A d S_{4}$.

Non-AdS backgrounds and harmonic functions. Non- $A d S_{4}$ backgrounds are also of interest because they are related to non-conformal field theories. Therefore it is of interest to find harmonic functions which are functions not only of the radius $r$, but also of other co-ordinates of the internal space.

We will now give here a simple way to construct non-trivial harmonic functions. Let us notice that all the four-dimensional quaternion Kahler orbifolds that we have constructed have two commuting Killing vectors which also preserve the one-forms $\omega_{-}^{i}$. This vector also preserves the Kahler triplet $d \bar{J}=$ $d \omega_{-}+\omega_{-} \wedge \omega_{-}$. Consequently they preserve the HyperKahler triplet (2.28) for the corresponding Swann fibration. Such vectors are therefore Killing and tri-holomorphic (thus tri-Hamiltonian). For any eight-dimensional hyperKahler metric with two commuting Killing vectors there exists a co-ordinate system which takes the form [35]

$$
g_{8}=U_{i j} d x^{i} \cdot d x^{j}+U^{i j}\left(d t_{i}+A_{i}\right)\left(d t_{j}+A_{j}\right)
$$

$\left(U_{i j}, A_{i}\right)$ being solutions of the generalized monopole equation

$$
\begin{aligned}
F_{x_{\mu}^{i} x_{\nu}^{j}} & =\epsilon_{\mu \nu \lambda} \nabla_{x_{\lambda}^{i}} U_{j}, \\
\nabla_{x_{\lambda}^{i}} U_{j} & =\nabla_{x_{\lambda}^{j}} U_{i}, \\
U_{i} & =\left(U_{i 1}, U_{i 2}\right) .
\end{aligned}
$$

The co-ordinates $\left(x_{i}^{1}, x_{i}^{2}\right)$ with $i=1,2,3$ are the momentum maps of the triholomorphic vector fields $\partial / \partial \theta$ and $\partial / \partial \varphi$, but we do not need to go in further details. In the momentum map system the 11-dimensional supergravity 
solution reads

$$
\begin{aligned}
g_{11} & =H^{-2 / 3} g_{2,1}+H^{1 / 3}\left[U_{i j} d x^{i} \cdot d x^{j}+U^{i j}\left(d t_{i}+A_{i}\right)\left(d t_{j}+A_{j}\right)\right], \\
F & = \pm \omega\left(E^{2,1}\right) \wedge d H^{-1},
\end{aligned}
$$

and the harmonic condition on $H$ is expressed as

$$
U^{i j} \partial_{i} \cdot \partial_{j} H=0
$$

All the Swann hyperKahler cones that we have presented are toric, and therefore they can be expressed as

$$
g_{8}=d r^{2}+r^{2} g_{7}=U_{i j} d x^{i} \cdot d x^{j}+U^{i j}\left(d t_{i}+A_{i}\right)\left(d t_{j}+A_{j}\right) .
$$

Let us recall that, as a consequence of (4.18), we have that $\partial_{i} \cdot \partial_{j} U_{i j}=0$, which implies that $U^{i j} \partial_{i} \cdot \partial_{j} U_{i j}=0$. This means that any entry $U_{i j}$ is a harmonic function over the hyperKahler cone. The matrix $U^{i j}$ is determined by the relation $U^{i j}=g_{8}\left(\partial_{i}, \partial_{j}\right)$, and the inverse matrix $U_{i j}$ will give us three independent non-trivial harmonic functions for the internal space in consideration.

As an example we can consider the cone $g_{8}=d r^{2}+r^{2} g_{7}, g_{7}$ being the tri-Sasaki metric corresponding to the AdS-Taub-Nut solution (4.1). For this cone we have that

$$
\begin{aligned}
U^{\widetilde{t t}} & =r^{2}(\widetilde{r}-N)^{2}+r^{2} V(\widetilde{r}), \\
U^{\widetilde{\phi} \widetilde{\phi}} & =4 N^{2} r^{2} V(\widetilde{r}) \cos ^{2} \widetilde{\theta}+\left(\widetilde{r}^{2}-N^{2}\right) r^{2} \sin ^{2} \widetilde{\theta} \\
U^{\widetilde{t \phi}} & =-2 N r^{2} V(\widetilde{r}) \cos \widetilde{\theta}+r^{2}(\widetilde{r}-N) g(\widetilde{r}) \cos \widetilde{\theta} .
\end{aligned}
$$

By defining $\Delta=\widetilde{U^{\widetilde{t t}}} U^{\widetilde{\phi} \widetilde{\phi}}-\left(\widetilde{U^{\widetilde{\phi}}}\right)^{2}$ we obtain the following harmonic functions

$$
U_{\widetilde{t t}}=\frac{U^{\widetilde{\phi} \widetilde{\phi}}}{\Delta}, \quad U_{\widetilde{\phi} \widetilde{\phi}}=-\frac{U^{\widetilde{t t}}}{\Delta}, \quad U_{\widetilde{t \phi}}=\frac{U^{\widetilde{t} \widetilde{\phi}}}{\Delta} .
$$

In the $S^{4}$ manifold limit we obtain more simple expressions, namely

$$
\begin{aligned}
U_{\widetilde{\phi} \widetilde{\phi}} & =-\frac{1}{r^{2}}\left(\frac{1}{\sin ^{2} \widetilde{\theta}+\sin ^{2} \widetilde{\rho} \cos ^{2} \widetilde{\theta}-\sin ^{4} \widetilde{\rho} \cos ^{2} \widetilde{\theta}}\right), \\
U_{\widetilde{\phi} \widetilde{t}} & =\frac{1}{r^{2}}\left(\frac{\sin ^{2} \widetilde{\theta}+\sin ^{2} \widetilde{\rho} \cos ^{2} \widetilde{\theta}}{\sin ^{2} \widetilde{\theta}+\sin ^{2} \widetilde{\rho} \cos ^{2} \widetilde{\theta}-\sin ^{4} \widetilde{\rho} \cos ^{2} \widetilde{\theta}}\right) \\
U_{\widetilde{t t}} & =\frac{1}{r^{2}}\left(\frac{\sin ^{2} \widetilde{\rho} \cos \widetilde{\theta}}{\sin ^{2} \widetilde{\theta}+\sin ^{2} \widetilde{\rho} \cos ^{2} \widetilde{\theta}-\sin ^{4} \widetilde{\rho} \cos ^{2} \widetilde{\theta}}\right)
\end{aligned}
$$


For the AdS-Kerr-Newman case (4.3) we have

$$
\begin{aligned}
U^{\widetilde{t t}}= & r^{2} \frac{c(\widetilde{r}) d(\widetilde{r})}{\widetilde{r}^{2}-a^{2} \cos \widetilde{\theta}^{2}}+a^{2} r^{2} \frac{f(\widetilde{\theta}) \sin ^{2} \widetilde{\theta}}{\widetilde{r}^{2}-a^{2} \cos ^{2} \widetilde{\theta}}+r^{2} e^{2}(\widetilde{r}, \widetilde{\theta}), \\
U^{\widetilde{t \phi}}= & r^{2} \frac{c(\widetilde{r}) d(\widetilde{r})}{\widetilde{r}^{2}-a^{2} \cos \widetilde{\theta}^{2}} \frac{a \sin ^{2} \widetilde{\theta}}{\Xi}+a r^{2} \frac{f(\widetilde{\theta}) \sin ^{2} \widetilde{\theta}}{\widetilde{r}^{2}-a^{2} \cos ^{2} \widetilde{\theta}} \frac{c(\widetilde{r})}{\Xi}+r^{2} \frac{W(\widetilde{r}, \widetilde{\theta})}{\Xi}, \\
U^{\widetilde{\phi} \widetilde{\phi}}= & r^{2} \frac{c(\widetilde{r}) d(\widetilde{r})}{\widetilde{r}^{2}-a^{2} \cos \widetilde{\theta}^{2}} \frac{a^{2} \sin ^{4} \widetilde{\theta}}{\Xi^{2}}+r^{2} \frac{f(\widetilde{\theta}) \sin ^{2} \widetilde{\theta}}{\widetilde{r}^{2}-a^{2} \cos ^{2} \widetilde{\theta}} \frac{c^{2}(\widetilde{r})}{\Xi^{2}} \\
& +r^{2} \frac{W^{2}(\widetilde{r}, \widetilde{\theta})}{\Xi^{2} e^{2}(\widetilde{r}, \widetilde{\theta})}+r^{2} \frac{f(\widetilde{\theta}) c(\widetilde{r}) d(\widetilde{r})}{e^{2}(\widetilde{r}, \widetilde{\theta})} \frac{\sin ^{2} \widetilde{\theta}}{\Xi^{2}}
\end{aligned}
$$

and again, the three functions $U^{i j} / \Delta$ are harmonic functions over the internal hyperKahler space. Notice that $\Delta \sim r^{4}$ and therefore all these harmonic functions depend on $r$ as $1 / r^{2}$. Finally, for the Swann metrics fibred over the toric orbifolds (3.28) we find

$$
U_{\alpha \alpha}=\frac{F}{r^{2}}\left(\frac{1}{2} F+\rho F_{\rho}\right), \quad U_{\alpha \beta}=\frac{\rho F_{\eta} F}{r^{2}}, \quad U_{\beta \beta}=\frac{F}{r^{2}}\left(\frac{1}{2} F-\rho F_{\rho}\right),
$$

are also harmonic functions over the cone, depending on a solution $F$ of a linear differential equation. All these harmonic functions provide non- $A d S_{4}$ horizon limits.

\section{Gamma deformations of supergravity backgrounds}

\subsection{Deformations of 11-supergravity solutions}

Let us describe in more detail the $S L(2, R)$ solution-generating technique sketched in the introduction. This technique was applied in order to find the dual of marginal deformed field theories in [12]. One usually starts with a solution of the 11-dimensional supergravity with $U(1) \times U(1) \times U(1)$ isometry. Any of such solutions can be written in the generic form

$$
\begin{aligned}
g_{11} & =\Delta^{1 / 3} M_{a b} D \alpha_{a} D \alpha_{b}+\Delta^{-1 / 6} \widetilde{g}_{\mu \nu} d x^{\mu} d x^{\nu} \\
C_{3} & =C D \alpha_{1} \wedge D \alpha_{2} \wedge D \alpha_{3}+C_{1(a b)} \wedge D \alpha_{a} \wedge D \alpha_{b}+C_{2(a)} \wedge D \alpha_{a}+C_{(3)},
\end{aligned}
$$

with the indices $a, b=1,2,3$ are associated to three co-ordinates $\alpha_{1}, \alpha_{2}$ and $\alpha_{3}$. The metric and the field $C_{3}$ does not depend on these co-ordinates and the greek indices $\mu, \nu$ run over the remaining eight-dimensional coordinates. We have introduced the covariant derivative $D \alpha_{i}=d \alpha_{i}+A_{i}, A_{i}$ being a triplet of $\alpha_{i}$-independent one-forms. The expression (5.13) possesses 
a manifest $S L(3, R)$ symmetry for which the co-ordinates $\alpha_{i}$ of (5.13) and the tensor fields $M$ and $A_{i}$ have the following transformation law

$$
\begin{aligned}
&\left(\begin{array}{l}
\alpha_{1} \\
\alpha_{2} \\
\alpha_{3}
\end{array}\right)^{\prime}=\left(\Lambda^{T}\right)^{-1}\left(\begin{array}{l}
\alpha_{1} \\
\alpha_{2} \\
\alpha_{3}
\end{array}\right), \\
& M^{\prime}=\Lambda M \Lambda^{T} \quad\left(\begin{array}{l}
A_{1} \\
A_{2} \\
A_{3}
\end{array}\right)=\left(\Lambda^{T}\right)^{-1}\left(\begin{array}{l}
A_{1} \\
A_{2} \\
A_{3}
\end{array}\right) . \\
&\left(\begin{array}{l}
C_{23 \mu} \\
C_{31 \mu} \\
C_{12 \mu}
\end{array}\right) \rightarrow\left(\Lambda^{T}\right)^{-1}\left(\begin{array}{l}
C_{23 \mu} \\
C_{31 \mu} \\
C_{12 \mu}
\end{array}\right), \quad\left(\begin{array}{l}
C_{1 \mu \nu} \\
C_{2 \mu \nu} \\
C_{3 \mu \nu}
\end{array}\right) \rightarrow \Lambda\left(\begin{array}{l}
C_{1 \mu \nu} \\
C_{2 \mu \nu} \\
C_{3 \mu \nu}
\end{array}\right) .
\end{aligned}
$$

The full isometry group of 11-dimensional supergravity compactified on a three torus is $S L(3, R) \times S L(2, R)$. The $S L(3, R)$ group leaves the background (5.13) unaltered. Following [12] and [108] we will deform these $T^{3}$ invariant backgrounds by an element of $S L(2, R)$. This deformation is a solution-generating technique which does not leave the background unchanged, but gives new supergravity backgrounds. We find convenient to define a complex parameter $\tau=C+i \Delta^{1 / 2}$ which, under the $S L(2, R)$ action is transformed as

$$
\tau \longrightarrow \frac{a \tau+b}{c \tau+d} ; \quad \Lambda=\left(\begin{array}{ll}
a & b \\
c & d
\end{array}\right) \in S L(2, R) .
$$

The eight-dimensional metric $g_{\mu \nu}$ and the tensor $C_{2}$ are invariant under this action. The tensor $C_{(1) a b \mu}$ and $A_{a \mu}$ form a doublet in a similar way that the RR and NSNS two-form fields do in IIB supergravity, their transformation law is

$$
B^{a}=\left(\begin{array}{c}
2 A_{a} \\
-\epsilon^{a b c} C_{(1) b c}
\end{array}\right), \quad B_{a} \longrightarrow \Lambda^{-T} B_{a} .
$$

The field strength $C_{3}$ also forms a doublet with its magnetic dual with transformation law

$$
H=\left(\begin{array}{c}
F_{4} \\
\Delta^{\frac{-1}{2}} *_{8} F_{4}+C_{(0)} F_{4}
\end{array}\right), \quad H \longrightarrow \Lambda^{-T} H,
$$

the Hodge operation being taken with respect to the eight-dimensional metric $\widetilde{g}$. As we discussed in the introduction, this transformation deforms the original metric (5.13) and the deformed metric will be regular only with elements of the form [12]

$$
\Lambda=\left(\begin{array}{ll}
1 & 0 \\
\gamma & 1
\end{array}\right) \in S L(2, R)
$$

which constitute a subgroup called $\gamma$-transformations. We will be concerned with such transformations in the following. 
If the fields $C, C_{1}$ and $C_{2}$ are zero, it follows that $A_{i}$ and $\widetilde{g}_{\mu \nu}$ are unchanged by a $\gamma$-transformation and $C_{1}$ and $C_{2}$ remains zero. The deformation then gives the new fields

$$
\Delta^{\prime}=G^{2} \Delta, \quad C^{\prime}=-\gamma G \Delta, \quad G=\frac{1}{1+\gamma^{2} \Delta} .
$$

By inspection of the transformation rule (5.6) it follows that

$$
F_{4}^{\prime}=F_{4}-\gamma \Delta^{-1 / 2} *_{8} F_{4}-\gamma d\left(G \Delta D \alpha_{1} \wedge D \alpha_{2} \wedge D \alpha_{3}\right) .
$$

The $\gamma$-deformed 11-dimensional metric results [108]

$$
g_{11}=G^{-1 / 3}\left(G \Delta^{1 / 3} M_{a b} D \alpha_{a} D \alpha_{b}+\Delta^{-1 / 6} \widetilde{g}_{\mu \nu} d x^{\mu} d x^{\nu}\right) .
$$

Note that if the initial four-form $F_{4}$ was zero, then from the last term in (5.9) a non-trivial flux is obtained in the deformed background.

\subsection{A family of deformed backgrounds}

As we have already mentioned, any eight-dimensional Ricci flat metric $g_{8}$ can be extended to a 11-dimensional supergravity solution of the form

$$
\begin{aligned}
& g_{11}=H^{-2 / 3}\left(-d t^{2}+d x^{2}+d y^{2}\right)+H^{1 / 3} g_{8} \\
& C^{3}= \pm H^{-1} d x \wedge d y \wedge d t, \quad F^{4}= \pm d x \wedge d y \wedge d t \wedge d H^{-1}
\end{aligned}
$$

$H$ being a harmonic function over $g_{8}$. If the metric $g_{8}$ is a cone then we can re-express the 11-dimensional metric as

$$
g_{11}=H^{-2 / 3}\left(-d t^{2}+d x^{2}+d y^{2}\right)+H^{1 / 3}\left(d r^{2}+r^{2} g_{7}\right)
$$

$g_{7}$ being an Einstein metric. If $g_{8}$ is hyperKahler then $g_{7}$ will be tri-Sasaki; if $g_{8}$ is of $\operatorname{Spin}(7)$ holonomy with cohomogeneity one, then $g_{7}$ will be of weak $G_{2}$ holonomy. We have constructed a whole family of tri-Sasaki metrics in (4.1), (4.3) and (4.4). In addition, the replacement $g_{q} \rightarrow 5 g_{q}$ in all these expressions give a family of metrics with weak $G_{2}$ holonomy. All these metrics possess three commuting Killing vectors, namely $\partial_{\tau}, \partial_{\alpha}$ and $\partial_{\beta}$. Therefore they are suitable to apply the $S L(2, R)$ solution-generating technique described previously. The corresponding 11-dimensional supergravity 
background with three commuting isometries is

$$
\begin{aligned}
g_{11}= & H^{-2 / 3}\left(-d t^{2}+d x^{2}+d y^{2}\right)+H^{1 / 3} d r^{2}+H^{1 / 3} r^{2}\left(\left(g_{7}\right)_{\widetilde{t t}} d \widetilde{t}^{2}+\left(g_{7}\right)_{\widetilde{t \phi}} d \widetilde{t}\right. \\
& \otimes d \widetilde{\phi}+\left(g_{7}\right)_{\widetilde{t} \tau} d \widetilde{t} \otimes d \tau+\left(g_{7}\right)_{\widetilde{\phi} \widetilde{\phi}} d \widetilde{\phi}^{2}+\left(g_{7}\right)_{\widetilde{\phi} \tau} d \widetilde{\phi} \otimes d \tau+\left(g_{7}\right)_{\tau \tau} d \tau^{2} \\
& \left.+Q_{\widetilde{t}} \widetilde{d t}+Q_{\widetilde{\phi}} d \widetilde{\phi}+Q_{\tau} d \tau+\widetilde{H}\right) \\
C^{3}= & \pm H^{-1} d x \wedge d y \wedge d t, \quad F^{4}= \pm d x \wedge d y \wedge d t \wedge d H^{-1}
\end{aligned}
$$

where we have defined

$$
\begin{aligned}
Q_{\widetilde{t}}= & \left(g_{7}\right)_{\widetilde{t} \theta} d \theta, \quad Q_{\widetilde{\phi}}=\left(g_{7}\right)_{\widetilde{\phi} \theta} d \theta, \quad Q_{\tau}=\left(g_{7}\right)_{\tau \varphi} d \varphi+\left(g_{7}\right)_{\tau \widetilde{\theta}} d \widetilde{\theta}+\left(g_{7}\right)_{\tau \widetilde{r}} d \widetilde{r} \\
\widetilde{H}= & d \theta^{2}+d \varphi^{2}+\left(g_{7}\right)_{\widetilde{\theta} \varphi} d \widetilde{\theta} \otimes d \varphi+\left(g_{7}\right)_{\widetilde{r} \widetilde{r}} d \widetilde{r}^{2}+\left(g_{7}\right)_{\widetilde{r} \widetilde{\theta}} d \widetilde{r} \otimes d \widetilde{\theta} \\
& +\left(g_{7}\right)_{\widetilde{r} \varphi} d \widetilde{r} \otimes d \varphi+g_{\widetilde{\theta} \widetilde{\theta}} d \widetilde{\theta}^{2} .
\end{aligned}
$$

Under the replacement $g_{q} \rightarrow 5 g_{q}$ we will obtain a new supergravity solution which is fibred over a weak $G_{2}$ holonomy space. The solution-generating technique applies to both cases exactly in the same manner, but the dualfield theories will possess different number of supercharges. Let us note that if the harmonic function is selected to be

$$
H(r)=1+\frac{2^{5} \pi^{2} N l_{p}^{6}}{r^{6}} .
$$

then the near horizon limit will be

$$
g_{11}=g_{A d S}+g_{7} .
$$

But we can consider backgrounds with other horizon limits by considering harmonic functions such as those constructed in (4.21) to (4.24).

Our task now is to find a local co-ordinate system for which (5.11) takes the manifest $T^{3}$ symmetric form

$$
g_{11}=\Delta^{1 / 3} M_{a b} D \phi_{a} D \phi_{b}+\Delta^{-1 / 6} \widetilde{g}_{\mu \nu} d x^{\mu} d x^{\nu}
$$

with the indices $a, b=1,2,3$ associated to the isometries $\phi_{1}=\widetilde{t}, \phi_{2}=\widetilde{\phi}$ and $\phi_{3}=\tau$ and the greek indices $\mu, \nu$ running over the remaining eightdimensional co-ordinates. We need to introduce the following quantities

$$
\begin{aligned}
\left(\begin{array}{l}
A_{1} \\
A_{2} \\
A_{3}
\end{array}\right) & =\left(g_{7}\right)^{a b}\left(\begin{array}{l}
Q_{\widetilde{t}} \\
Q_{\widetilde{\phi}} \\
Q_{\tau}
\end{array}\right) \\
h & =\widetilde{H}-r^{2} H^{1 / 3}\left(g_{7}\right)_{a b} A_{a} A_{b} .
\end{aligned}
$$

With the help of these quantities it is not difficult to check that the metric takes the form

$$
g_{11}=g_{8}+H^{1 / 3} r^{2}\left(g_{7}\right)_{a b}\left(d \phi_{a}+A_{a}\right)\left(d \phi_{b}+A_{b}\right)
$$


$g_{8}$ being given by

$$
g_{8}=H^{-2 / 3}\left(-d t^{2}+d x^{2}+d y^{2}\right)+H^{1 / 3}\left(d r^{2}+r^{2} h\right)
$$

By further defining

$$
\begin{gathered}
\Delta=\left[\operatorname{det} \Omega^{a b}\right], \quad \widetilde{g}=\Delta^{1 / 6} g_{8} \\
D \phi_{a}=d \phi_{a}+A_{a}, \quad \Omega^{a b}=r^{2} H^{1 / 3}\left(g_{7}\right)_{a b}, \quad M_{a b}=\frac{\Omega^{a b}}{\operatorname{det}\left(\Omega^{a b}\right)^{1 / 3}} .
\end{gathered}
$$

The metric take the desired form with manifest $S L(3, R)$ symmetry

$$
g_{11}=\Delta^{1 / 3} M_{a b} D \phi_{a} D \phi_{b}+\Delta^{-1 / 6} \widetilde{g}_{\mu \nu} d x^{\mu} d x^{\nu}
$$

By using these quantities together with formulae (5.8) to (5.10) we obtain the following deformed backgrounds.

For the orbifolds (4.8) we have that

$$
\begin{gathered}
Q_{\alpha}=\left(g_{7}\right)_{\alpha \theta} d \theta+\left(g_{7}\right)_{\alpha \varphi} d \varphi, \quad Q_{\beta}=\left(g_{7}\right)_{\beta \varphi} d \varphi \\
Q_{\phi}=\left(g_{7}\right)_{\phi \varphi} d \varphi+\left(g_{7}\right)_{\phi \eta} d \eta+\left(g_{7}\right)_{\phi \rho} d \rho \\
\widetilde{H}=d \theta^{2}+d \varphi^{2}+\left(g_{7}\right)_{\theta \eta} d \theta \otimes d \eta+\left(g_{7}\right)_{\rho \rho} d \rho^{2}+\left(g_{7}\right)_{\rho \eta} d \rho \otimes d \eta+\left(g_{7}\right)_{\eta \eta} d \eta^{2} .
\end{gathered}
$$

The explicit form of the matrix $\Omega_{a b}$ turns out to be

$$
\Omega_{a b}=r^{2} H^{1 / 3}\left(\begin{array}{ccc}
1 & -\frac{2 \sqrt{\rho}}{F} \sin \theta \cos \varphi+\frac{2 \eta}{\sqrt{\rho} F} \cos \theta & -\frac{2}{\sqrt{\rho} F} \cos \theta \\
-\frac{2 \sqrt{\rho}}{F} \sin \theta \cos \varphi+\frac{2 \eta}{\sqrt{\rho} F} \cos \theta & \left(g_{q}\right)_{\alpha \alpha}+\frac{\left(1+\rho^{2}\right)}{\rho F^{2}} & \left(g_{q}\right)_{\alpha \beta}+\frac{2 \eta}{\rho F^{2}} \\
-\frac{2}{\sqrt{\rho} F} \cos \theta & \left(g_{q}\right)_{\alpha \beta}+\frac{2 \eta}{\rho F^{2}} & \left(g_{q}\right)_{\beta \beta}+\frac{1}{\rho F^{2}}
\end{array}\right),
$$

$g_{q}$ being the quaternion Kahler metric defined in (3.28). All these quantities are expressed in terms of a single eigenfunction $F$ of the laplacian operator in the hyperbolic two-dimensional space. By using the formulae (5.8), (5.9) and (5.10) we obtain directly the deformed backgrounds, as before.

\subsection{Explicit formulae for the spherical case}

It will be instructive to repeat this procedure to the background (5.31) fibred over $S^{4}$. We need to define the relevant quantities first. From the definition $\Omega^{a b}=g_{a b}$ being $a, b=1,2,3$ and $\phi_{1}=\widetilde{t}, \phi_{2}=\widetilde{\phi}$ and $\phi_{3}=\tau$, we find the 
following toric fibre metric

$$
\begin{gathered}
\Omega^{\widetilde{t t}}=\Omega^{\widetilde{\phi} \widetilde{\phi}}=\Omega^{\tau \tau}=1, \quad \Omega^{\widetilde{t} \widetilde{\phi}}=\sin \widetilde{\rho} \cos \widetilde{\theta}, \quad \Omega^{\widetilde{t \tau}}=-\sin \widetilde{\rho} \sin \theta \cos \varphi, \\
\Omega^{\widetilde{\phi} \tau}=-\sin \theta(\sin \varphi \cos \widetilde{\rho} \sin \widetilde{\theta}+\cos \varphi \cos \widetilde{\theta}) .
\end{gathered}
$$

The determinant $\Delta=\operatorname{det} \Omega^{a b}$ of this matrix is

$$
\begin{aligned}
\Delta= & -\sin \widetilde{\rho} \sin \theta \cos \varphi[\sin \widetilde{\rho} \cos \widetilde{\theta} \sin \theta(\sin \varphi \cos \widetilde{\rho} \sin \widetilde{\theta}+\cos \varphi \cos \widetilde{\theta}) \\
& +\sin \widetilde{\rho} \sin \theta \cos \varphi]+\left[1-\sin ^{2} \theta(\sin \varphi \cos \widetilde{\rho} \sin \widetilde{\theta}+\cos \varphi \cos \widetilde{\theta})^{2}\right] \\
& -\sin \widetilde{\rho} \cos \widetilde{\theta}(\sin \widetilde{\rho} \cos \widetilde{\theta}+\sin \widetilde{\rho} \sin \theta \cos \varphi) .
\end{aligned}
$$

From (5.19) and (5.20) we define the matrix $M_{a b}=\Omega^{a b} / \Delta^{1 / 3}$ with unit determinant. Also, from the definition $\Omega_{a b}=g^{a b}$ of the inverse matrix we obtain that

$$
\begin{aligned}
& \Omega_{\widetilde{t t}}=\frac{1}{\Delta}\left(1-\sin ^{2} \theta(\sin \varphi \cos \widetilde{\rho} \sin \widetilde{\theta}+\cos \varphi \cos \widetilde{\theta})^{2}\right), \\
& \Omega_{\widetilde{\phi} \widetilde{\phi}}=\frac{1}{\Delta}\left(1+\sin ^{2} \widetilde{\rho} \sin ^{2} \theta \cos ^{2} \varphi\right), \quad \Omega_{\tau \tau}=\frac{1}{\Delta}\left(1-\sin ^{2} \widetilde{\rho} \cos ^{2} \widetilde{\theta}\right), \\
& \Omega_{\widetilde{t \phi}}=\frac{1}{\Delta}\left(\sin \widetilde{\rho} \cos \widetilde{\theta}-\sin ^{2} \theta \sin \widetilde{\rho} \cos \varphi(\sin \varphi \cos \widetilde{\rho} \sin \widetilde{\theta}+\cos \varphi \cos \widetilde{\theta})\right), \\
& \Omega_{\widetilde{t \tau}}=\frac{1}{\Delta}(\sin \theta \sin \widetilde{\rho} \cos \varphi-\sin \widetilde{\rho} \cos \widetilde{\theta} \sin \theta(\sin \varphi \cos \widetilde{\rho} \sin \widetilde{\theta}+\cos \varphi \cos \widetilde{\theta})), \\
& \Omega_{\widetilde{\phi} \tau}=\frac{1}{\Delta}\left(\sin \theta \sin ^{2} \widetilde{\rho} \cos \widetilde{\theta} \cos \varphi-\sin \theta(\sin \varphi \cos \widetilde{\rho} \sin \widetilde{\theta}+\cos \varphi \cos \widetilde{\theta})\right) .
\end{aligned}
$$

The one-forms $Q_{i}$ are

$$
\begin{gathered}
Q_{\widetilde{t}}=\sin \widetilde{\rho} \sin \varphi d \theta, \quad Q_{\widetilde{\phi}}=(\cos \widetilde{\theta} \sin \varphi-\cos \widetilde{\rho} \sin \widetilde{\theta} \cos \varphi) d \theta \\
Q_{\tau}=-\cos \theta(d \varphi+\cos \widetilde{\rho} d \widetilde{\theta})
\end{gathered}
$$

With the help of (5.21) and (5.22) we define the one-forms $A_{a}$ and the covariant derivative $D_{a}$ by

$$
A_{a}=\Omega_{a b} Q_{b}, \quad D \phi_{a}=d \phi_{a}+A_{a} .
$$

The metric $\widetilde{H}$ defined in (5.12) is

$$
\widetilde{H}=d \theta^{2}+d \varphi^{2}+d \widetilde{\rho}^{2}+d \widetilde{\theta}^{2}-2 \cos \widetilde{\rho} d \widetilde{\theta} \otimes d \varphi,
$$

and therefore the metric in (5.2) reads

$$
h=\widetilde{H}-\left(g_{7}\right)_{a b} A_{a} A_{b} .
$$


With the help of formulae (5.13) to (5.17) we obtain the deformed background, the result is

$$
\begin{aligned}
& g_{d}=\left(1+\gamma^{2} \Delta\right)^{1 / 3}\left(g_{11}-\frac{\gamma^{2} \Delta}{1+\gamma^{2} \Delta} \Omega_{a b} Q_{a} Q_{b}\right) \\
& C=-\frac{k \sin u}{3} \sinh ^{3} \rho d t \wedge d u \wedge d v-\frac{\gamma \Delta}{1+\gamma^{2} \Delta} D \widetilde{t} \wedge D \widetilde{\phi} \wedge D \tau,
\end{aligned}
$$

$g_{11}$ being the undeformed metric (5.31). Notice that (5.26) is explicit because all the quantities are defined by (5.20), (5.21) and (5.22). The procedure is completed.

We will consider IIB reductions of these backgrounds and their deformations in the appendix.

\subsection{Rotating supermembrane solutions}

We have presented an infinite family of 11-dimensional supergravity backgrounds possessing at least three commuting Killing vectors. These backgrounds are supposed to be dual to three-dimensional conformal field theories arising as the infrared limit of the world volume theory of $N$ coincident M2 branes located a the singularity of $M_{3} \times X_{8}$. Because the eightdimensional geometry is hyperKahler we expect $N=3$ supersymmetry in the superconformal field theory. But it is difficult to guess which is the explicit form of the dual-field theory and we are not attempting to obtain an explicit Lagrangian here. Nevertheless it has been suggested that the correspondence between semiclassical strings with high angular momentum and long operators can be generalized to membranes [20]. In the string case the configurations have energy proportional to the t' Hooft scale and thus are dual to operators with large dimensions [7]. For a rotating string in $A d S_{5}$ the difference between the energy and the spin depends logarithmically on the spin, therefore the dual operators should possess dimensions with the same dependence. The operators are twist operators that are responsible for violations of Bjorken scaling, and it has been shown that no corrections to the logarithmic behaviour appears in the strong coupling limit [8]. This correspondence has been generalized to membranes, in which the relation between the spin, the J-charges and the energy should be related to the anomalous dimensions of certain operators of the conformal field theory [20].

Therefore it is of interest to consider rotating membrane configurations over the supergravity backgrounds that we have constructed. Recall that 
the supermembrane action is given by

$$
\begin{aligned}
S= & -\frac{1}{2 \pi^{2} l_{11}^{3}} \int\left(\frac{(-\gamma)^{1 / 2}}{2}\left[\gamma_{i j} \frac{\partial X_{\mu}}{\partial \sigma_{i}} \frac{\partial X_{\nu}}{\partial \sigma_{j}} G_{\mu \nu}-1\right]\right. \\
& \left.+\epsilon_{i j k} \frac{\partial X_{\nu}}{\partial \sigma_{i}} \frac{\partial X_{\nu}}{\partial \sigma_{j}} \frac{\partial X_{\nu}}{\partial \sigma_{k}} C_{\mu \nu \rho}\right) d^{3} \sigma
\end{aligned}
$$

where $\sigma_{i}=(\tau, \sigma, \lambda)$ are the worldvolume co-ordinates, $\gamma_{i j}$ the worldvolume metric, $X_{\mu}$ is the then target space co-ordinate and $G_{\mu \nu}$ is the target metric. We have also the three-form $C_{\mu \nu \rho}$ and the corresponding field strength is $H=d C$. The equations of motion derived from (5.27) is

$$
\begin{aligned}
\gamma_{i j}= & \partial_{i} X_{\mu} \partial_{j} X_{\nu} G_{\mu \nu} \\
\partial_{i}\left((-\gamma)^{1 / 2} \gamma^{i j} \partial_{j} X^{\rho}\right)= & -(-\gamma)^{1 / 2} \gamma^{i j} \partial_{i} X^{\mu} \partial_{j} X^{\nu} \Gamma_{\mu \nu}^{\rho}(X) \\
& -\epsilon^{i j k} \partial_{i} X^{\mu} \partial_{j} X^{\nu} \partial_{k} X^{\sigma} H_{\mu \nu \sigma}^{\rho}(X) .
\end{aligned}
$$

The three diffeomorphisms of the action can be fixed by a gauge described by the constraints

$$
\begin{gathered}
\gamma_{0 \alpha}=\partial_{0} X^{\mu} \partial_{\alpha} X^{\nu} G_{\mu \nu}(X)=0 \\
\gamma_{00}+L^{2} \operatorname{det}\left[\gamma_{\alpha \beta}\right]=\partial_{0} X^{\mu} \partial_{0} X^{\nu} G_{\mu \nu}(X)+L^{2} \operatorname{det}\left[\partial_{\alpha} X^{\mu} \partial_{\beta} X^{\nu} G_{\mu \nu}(X)\right]=0,
\end{gathered}
$$

$L$ being a constant fixed by the equations of motion. By imposing the constraints $(5.27)$ to $(5.29)$ we obtain the following action in gauge fixed form

$$
\begin{aligned}
S= & \frac{1}{2(2 \pi)^{2} L l_{11}^{3}} \int\left(\partial_{0} X^{\mu} \partial_{0} X^{\nu} G_{\mu \nu}(X)-L^{2} \operatorname{det}\left[\partial_{\alpha} X^{\mu} \partial_{\beta} X^{\nu} G_{\mu \nu}(X)\right]\right. \\
& \left.+2 L \epsilon^{i j k} \partial_{i} X^{\mu} \partial_{j} X^{\nu} \partial_{k} X^{\rho} C_{\mu \nu \rho}(X)\right) d^{3} \sigma
\end{aligned}
$$

In Poincare co-ordinates the $A d S_{4}$ space is parameterized as

$$
g_{A d S}=-\cosh ^{2} \rho d t^{2}+d \rho^{2}+\sinh ^{2} \rho\left(d u^{2}+\sin ^{2} u d v^{2}\right) .
$$

and the 11-dimensional background becomes

$$
\begin{aligned}
\frac{1}{l_{11}^{2}} g_{11}^{2} & =-\cosh ^{2} \rho d t^{2}+d \rho^{2}+\sinh ^{2} \rho\left(d u^{2}+\sin ^{2} u d v^{2}\right)+r_{12} g_{7}, \\
F_{4} & =k \cosh \rho \sinh ^{2} \rho \sin u d t \wedge d \rho \wedge d u \wedge d v \\
C & =-\frac{k \sin u}{3} \sinh ^{3} \rho d t \wedge d u \wedge d v
\end{aligned}
$$

$r_{12}$ being the relative radius between the $A d S_{4}$ and the internal space and $k$ is a constant determined by the equation of motions. 
We will study now the case when the metric on $X_{7}$ is the tri-Sasaki metric fibred over the sphere $S^{4}$, the metric is given in (4.4). Our 11-dimensional background is

$$
\begin{aligned}
g_{11}= & -\cosh ^{2} \rho d t^{2}+d \rho^{2}+\sinh ^{2} \rho\left(d u^{2}+\sin ^{2} u d v^{2}\right) \\
& +(\cos \widetilde{\rho} \sin \widetilde{\theta} d \widetilde{\phi}-\cos \varphi d \theta-\sin \varphi \sin \theta d \tau)^{2} \\
& +(\cos \widetilde{\rho} d \widetilde{\theta}-d \varphi-\cos \theta d \tau)^{2} \\
& +(\sin \widetilde{\rho} d \widetilde{t}+\cos \widetilde{\theta} d \widetilde{\phi}+\sin \varphi d \theta-\cos \varphi \sin \theta d \tau)^{2} \\
& +\cos ^{2} \widetilde{\rho} d \widetilde{t}^{2}+d \widetilde{\rho}^{2}+\sin ^{2} \widetilde{\rho}\left(d \widetilde{\theta}^{2}+\sin ^{2} \widetilde{\theta} d \widetilde{\phi}^{2}\right)
\end{aligned}
$$

The first configuration is one rotating in the AdS background and for which the third direction is wrapped in the Reeb direction

$$
\begin{gathered}
\rho=\rho(\sigma), \quad t=k \widetilde{\tau}, \quad u=\frac{\pi}{2}, \quad v=\omega \widetilde{\tau}, \quad \theta=\frac{\pi}{2}, \quad \varphi=\frac{\pi}{2}, \\
\tau=\lambda \delta, \quad \widetilde{\rho}=\frac{\pi}{2}, \quad \widetilde{\theta}=\frac{\pi}{2}, \quad \widetilde{\phi}=\nu_{\phi} \widetilde{\tau}, \quad \widetilde{t}=\nu_{t} \widetilde{\tau} .
\end{gathered}
$$

The second configuration we will analyse is one in which the membrane rotates in the internal space $(R$-charge) and the third direction is wrapped in AdS, namely

$$
\begin{gathered}
\rho=\rho(\sigma), \quad t=k \widetilde{\tau}, \quad u=\frac{\pi}{2}, \quad v=\lambda \delta, \quad \theta=\frac{\pi}{2}, \quad \varphi=\frac{\pi}{2}, \\
\tau=\nu \widetilde{\tau}, \quad \widetilde{\rho}=\frac{\pi}{2}, \quad \widetilde{\theta}=\frac{\pi}{2}, \quad \widetilde{\phi}=\nu_{\phi} \widetilde{\tau}, \quad \widetilde{t}=\nu_{t} \widetilde{\tau} .
\end{gathered}
$$

For the first configuration by selecting $L=1$ we have that

$$
\begin{aligned}
& g_{\mu \nu} \partial_{\sigma} X^{\mu} \partial_{\widetilde{\tau}} X^{\nu}=g_{\mu \nu} \partial_{\delta} X^{\mu} \partial_{\widetilde{\tau}} X^{\nu}=g_{\mu \nu} \partial_{\sigma} X^{\mu} \partial_{\delta} X^{\nu}=0 \\
& g_{\mu \nu} \partial_{\widetilde{\tau}} X^{\mu} \partial_{\widetilde{\tau}} X^{\nu}=-\kappa^{2} \cosh ^{2} \rho+\omega^{2} \sinh ^{2} \rho+\nu_{\phi}^{2}+\nu_{t}^{2} \\
& g_{\mu \nu} \partial_{\sigma} X^{\mu} \partial_{\sigma} X^{\nu}=\left(\frac{d \rho}{d \sigma}\right)^{2},
\end{aligned}
$$

and the equations of motion gives the further relation

$$
\frac{d \rho}{d \sigma}=\sqrt{-\kappa^{2} \cosh ^{2} \rho+\omega^{2} \sinh ^{2} \rho+\nu_{\phi}^{2}+\nu_{t}^{2}} .
$$

Inserting this equation into the action gives us

$$
S=-P \int d \widetilde{\tau} \int_{0}^{\rho_{0}} \sqrt{-\kappa^{2} \cosh ^{2} \rho+\omega^{2} \sinh ^{2} \rho+\nu_{\phi}^{2}+\nu_{t}^{2}} .
$$


We have that

$$
\begin{aligned}
& E=-\frac{\delta S}{\delta k} \sim \int_{0}^{\rho_{0}} \frac{\cosh ^{2} \rho}{\sqrt{-\kappa^{2} \cosh ^{2} \rho+\omega^{2} \sinh ^{2} \rho+\nu_{\phi}^{2}+\nu_{t}^{2}}} \\
& S=\frac{\delta S}{\delta \omega} \sim \int_{0}^{\rho_{0}} \frac{\sinh ^{2} \rho}{\sqrt{-\kappa^{2} \cosh ^{2} \rho+\omega^{2} \sinh ^{2} \rho+\nu_{\phi}^{2}+\nu_{t}^{2}}} .
\end{aligned}
$$

But the integral (5.33) is one of those appearing in [7], and we obtain from here that $E-S \sim \log S$, which is what we wanted to show.

For the other configuration we have that

$$
S=-P \int d \widetilde{\tau} \int_{0}^{\rho_{0}} \sinh \rho \sqrt{-\kappa^{2} \cosh ^{2} \rho+\nu^{2}+\nu_{\phi}^{2}+\nu_{t}^{2}} .
$$

In this case we have no place for spin; but, we have energy and $R$-charge angular momentum $J$

$$
E=-\frac{\delta S}{\delta k}, \quad J=\frac{\delta S}{\delta \nu} .
$$

From (5.34) it is obtained that for long membranes, we have $E=J+\cdots$

Let us consider now the rotating configuration (5.32) in the deformed background (5.26). For this configuration we have that $\Delta=1, \Omega^{a b}=1$ and $Q_{i}$ and $D \tilde{t} \wedge D \widetilde{\phi} \wedge D \tau$ are zero. This means that the effective metric that the membrane sees rotating over (5.26) or (5.31) is essentially the same. Thus the logarithmic behaviour of the difference $E-S$ is reproduced for the deformed background. We find this interesting because, while the undeformed background is a direct product of $A d S_{4}$ with a seven space, the deformation is not.

\section{Kahler-Einstein over Kahler-Einstein and other examples}

Till the moment have found an explicit expression for Kahler-Einstein metrics defined over the twistor space $Z$ of any four-dimensional quaternion Kahler space. We also have found the corresponding Einstein-Sassaki metrics and we have checked, in accordance with [79], that the eight-dimensional cone over them is hyperKahler. It is indeed a Swann metric. Thus such Einstein-Sasaki metrics admit three conformal Killing vectors and are triSasaki. This is different than other Kahler-Einstein spaces appearing in the literature, for which the Einstein-Sasaki metrics admit only two conformal Killing vectors. In this section we review some Kahler-Einstein metrics which are fibred over Kahler metrics of lower dimensions [66]. In general 
they correspond to Einstein-Sasaki metrics which are not tri-Sasaki. We consider their construction important, because they encode several known spaces appearing in the literature.

\subsection{The Pedersen-Poon Hamiltonian approach}

We consider a Kahler space $M$ with metric $g$, a Kahler form $\Omega$ and a complex structure $J$. We assume the presence of $n$ Killing vectors $\left(X_{1}, \ldots, X_{N}\right)$ for which $\mathcal{L}_{X_{i}} \Omega=0$ which means that the generalized torus $T^{n}$ act through holomorphic isometries over $M$. A holomorphic isometry is also Hamiltonian, i.e., $\mathcal{L}_{X_{i}} J=0$. The Killing vectors are linearly independent in a dense open set of $M$, and are isotropic, i.e., $\Omega(X, X)=0$. This implies that $J X$ is orthogonal to every component of $X$. From the relation

$$
\mathcal{L}_{X_{i}} \Omega=i_{X_{i}} d \Omega+d\left(i_{X_{i}} \Omega\right)=d\left(i_{X_{i}} \Omega\right)=0,
$$

$i_{X_{i}}$ being the contraction of the vector field $X_{i}$ with the two-form $\Omega$, it follows the existence of $N$ functions $z_{i}$, called momentum maps, defined through the relations

$$
d z_{i}=i_{X_{i}} \Omega
$$

The manifold $M$ can be viewed as a torus bundle over a real manifold of dimension $2 m-N, m$ being the complex dimension of $M$. By denoting the $N$ fibre co-ordinates as $\left(t_{1}, \ldots, t_{N}\right)$ it follows that the metric takes the form

$$
g=h+w_{i j} d z^{i} d z^{j}+\left(w^{-1}\right)_{i j}\left(d t_{i}+\theta_{i}\right)\left(d t_{j}+\theta_{j}\right)
$$

in the momentum map system, $\theta_{i}$ being certain one-forms defined over the base space $h$ of the bundle. The matrix $w_{i j}$ is symmetric and positively definite.

The manifold obtained by the quotient of $M$ by the torus $T^{N}$ is described by the co-ordinates $z_{i}$ and other complex co-ordinates $\xi_{\nu}$ with $\nu=1, \ldots$, $m-N$. The metric $h$ is $2(m-N)$ dimensional, but depends on the co-ordinates $z_{i}$ as evolution parameters. In other words $h$ is the metric on the quotient space of each level set of the momentum maps. Both the matrix $u$ and the base metric $h$ are in principle $z_{i}$ dependent and $t_{i}$ independent. The metric $h$ is Kahler [75] and therefore complex, and can be written in complex form

$$
h=h_{a b} d \xi^{a} d \bar{\xi}^{b}
$$

From the definition of $\Omega$ it follows directly that

$$
g\left(J X_{i}, X_{j}\right)=\Omega\left(X_{i}, X_{j}\right)=d z_{i}\left(X_{j}\right)
$$

and therefore

$$
-J\left(d t_{i}+\theta_{i}\right)=w_{i j} d z_{j} .
$$


This implies that

$$
i\left(d t_{i}+\theta_{i}\right)+w_{i j} d z_{j}
$$

are $(0,1)$ type forms. The metric $(6.2)$ can be expressed in complex form as

$$
g=h_{a b} d \xi^{a} d \bar{\xi}^{b}+\left(w^{-1}\right)_{i j} i\left[w_{i j} d z_{j}+i\left(d t_{i}+\theta_{i}\right)\right]\left[w_{i j} d z_{j}-i\left(d t_{i}+\theta_{i}\right)\right],
$$

and the corresponding Kahler form is

$$
\Omega=\Omega_{h}+d z_{i} \wedge\left(d t_{i}+\theta_{i}\right) .
$$

From the fact that $\Omega$ is closed, a differential system involving $u$, the Kahler metric $h_{a b}$ and $\theta_{i}$ is obtained. The resulting equation is

$$
d \theta_{k}=\frac{i}{2} \frac{\partial h_{a b}}{\partial z_{k}} d \xi^{a} \wedge d \bar{\xi}^{b}+i \frac{\partial w_{k l}}{\partial \xi^{k}} d z^{j} \wedge d \xi^{k}-i \frac{\partial w_{k l}}{\partial \bar{\xi}^{k}} d z^{j} \wedge d \bar{\xi}^{k},
$$

and the integrability condition $d\left(d \theta_{k}\right)=0$ is equivalent to the equation

$$
\frac{\partial^{2} h_{a b}}{\partial z_{i} \partial z_{j}}+\frac{\partial^{2} w_{i j}}{\partial \xi^{a} \partial \bar{\xi}^{b}}=0
$$

The constructed metric is Kahler. It will be also Einstein if

$$
\rho=\Lambda \Omega
$$

$\rho=\operatorname{Ric}(J \cdot, \cdot)=-i \bar{\partial} \partial \log \operatorname{det} g$ being the Ricci form of the metric $g$, and the scalar curvature will be $2 m \Lambda$. The resulting system for Kahler-Einstein metrics was worked out in [66]. By defining the function $u$ by

$$
u=\log \operatorname{det} h-\log \operatorname{det} w,
$$

and using that in a Kahler manifold $-i \bar{\partial} \partial=d J d$ it follows that the system (6.9) is equivalent to

$$
d(J d u)=A \Omega,
$$

where we have defined $A=-2 \Lambda$. By taking into account the expression of $\Omega(6.6)$, the following differential system is obtained from the last condition

$$
\begin{gathered}
4 \frac{\partial^{2} u}{\partial \xi_{\lambda} \partial \bar{\xi}_{\mu}}+\frac{\partial u}{\partial z_{k}}\left(w^{-1}\right)_{k l} \frac{\partial h_{\lambda \mu}}{\partial z_{l}}=A h_{\lambda \mu}, \\
\frac{\partial\left(\partial u / \partial z_{k}\left(w^{-1}\right)_{k l}\right)}{\partial \xi_{\lambda}}=0, \quad \frac{\partial\left(\partial u / \partial z_{k}\left(w^{-1}\right)_{k l}\right)}{\partial z_{i}}=A \delta_{i l} .
\end{gathered}
$$

The last equation implies that

$$
\frac{\partial u}{\partial z_{k}}\left(w^{-1}\right)_{k l}=A z_{l}+B .
$$


From all this discussion it follows that our toric Kahler-Einstein metrics are described by the system

$$
\begin{array}{r}
4 \frac{\partial^{2} u}{\partial \xi_{\lambda} \partial \bar{\xi}_{\mu}}+\left(A z_{l}+B_{l}\right) \frac{\partial h_{\lambda \mu}}{\partial z_{l}}=A h_{\lambda \mu}, \\
\frac{\partial u}{\partial z_{k}}\left(w^{-1}\right)_{k l}=A z_{l}+B_{l}, \\
\frac{\partial^{2} h_{a b}}{\partial z_{i} \partial z_{j}}+\frac{\partial^{2} w_{i j}}{\partial \xi^{a} \partial \bar{\xi}^{b}}=0 .
\end{array}
$$

These equations describe metrics with commuting Killing vectors. But in order to have a free torus action the co-ordinates $t_{k}$ should be periodically identified. This is achieved if the closed form

$$
d \theta_{k}=\frac{i}{2} \frac{\partial h_{a b}}{\partial z_{k}} d \xi^{a} \wedge d \bar{\xi}^{b}+i \frac{\partial w_{k l}}{\partial \xi^{k}} d z^{j} \wedge d \xi^{k}-i \frac{\partial w_{k l}}{\partial \bar{\xi}^{k}} d z^{j} \wedge d \bar{\xi}^{k}
$$

is an integral form for any $k$. In this case there will not be singularities if the co-ordinate $t_{k}$ is periodic.

In the $N=1$ case, i.e., when there is only one $U(1)$ holomorphic isometry, the system (6.12) is reduced to

$$
\begin{gathered}
4 \frac{\partial^{2} u}{\partial \xi_{\lambda} \partial \bar{\xi}_{\mu}}+\left(A z+B_{l}\right) \frac{\partial h_{\lambda \mu}}{\partial z}=A h_{\lambda \mu}, \\
\frac{\partial u}{\partial z_{k}}\left(w^{-1}\right)=A z+B, \\
\frac{\partial^{2} h_{a b}}{\partial z^{2}}+\frac{\partial^{2} w}{\partial \xi^{a} \partial \bar{\xi}^{b}}=0 .
\end{gathered}
$$

Following [66] it is known that the system can be simplified by imposing that the Kahler quotient metrics obtained from each set of levels are homothetic, i.e., $h=f(z) q$ being the metric $q$ independent on the $z$ co-ordinate. A further simplification is obtained if the length function $w$ is just a function of $z$. In this case it follows from (6.14) that $f(z)=C z+D C$ and $D$ being constants, and that

$$
-\frac{\partial^{2} \log \operatorname{det} q}{\partial \xi_{\lambda} \partial \xi_{\nu}}=k q_{\lambda \nu},
$$

the constant $k$ being defined by $4 k=B C-A D$. This means that $q$ is also a Kahler-Einstein metric with scalar curvature $4 n k$. The class $F_{k}$ reduces in this case to the Chern form of the Kahler-Einstein base, which takes values $2 \pi Z$ for any Kahler-Einstein metric. Thus the metric that we are presenting is defined on a circle bundle. The length function $w$ is obtained 
from the second (6.12). The result shows that there is no loss of generality in selecting $C=1$ and $D=0$; the solution is given by

$$
w=\frac{z^{n}}{p z^{n+2}+q z^{n+1}+s}
$$

where $p=A / n+2, q=B / n+1$ and $s$ another constant. The local form of this subfamily of metrics is

$$
g^{\prime}=z q+w d z^{2}+\frac{(d \tau+A)^{2}}{w} .
$$

$A$ being given by

$$
d A=\Omega_{q} .
$$

The Kahler form of the new metric is simply

$$
\Omega^{\prime}=z \Omega_{q}+d z \wedge(d \tau+A),
$$

and can also be expressed as

$$
\Omega^{\prime}=d(z A)+d z \wedge d \tau=d A^{\prime}, \quad A^{\prime}=z A+z d \tau .
$$

The co-ordinate $z$ plays a role of a momentum map of the isometry $\partial_{\tau}$.

\subsection{Complete metrics}

Let us consider a $2 n$-dimensional Kahler-Einstein metric with sectional curvature normalized to one. This condition together with $B=4 \kappa$ fix the value $B=n+1$. The metric (6.16) takes in this case the following form

$$
g_{6}=\frac{d r^{2}}{V}+\frac{r^{2}}{4} V(d t+A)^{2}+\frac{r^{2}}{4} g_{f s},
$$

$V$ being given by

$$
V=1-\left(\frac{a}{r}\right)^{2 n+2}-\frac{\Lambda}{2(n+2)} r^{2} .
$$

There is an apparent singularity at the zeroes $r_{0}$ of $V$. Nevertheless such singularities can be removed for certain values of the parameters of the metric [36-66]. If $\Lambda>0$ then the metric will be complete if and only if $a=0$ and the base space is $C P(n)$ with its canonical metric. In this case the total space will be $C P(n+1)$ with the Fubbini-Study metric [36]. If instead $\Lambda<0$ there exists another complete metrics for certain values of the parameters [66]. This is seen as follows. Let us consider the fibre metric

$$
g_{f}=\frac{d r^{2}}{V}+\frac{r^{2}}{4} V d t^{2},
$$

and let us introduce the radial co-ordinate $R^{2}=r^{2} V$. The fibre metric has apparent singularities at the zeroes of $V$ and the co-ordinate $R$ tends to zero 
near the singularities. By differentiating (6.20) it is obtained that

$$
\frac{d V}{d r}=\frac{2}{r} U, \quad U=(n+1)\left(\frac{a}{r}\right)^{2 n+2}-\frac{\Lambda}{2(n+2)} r^{2},
$$

and in terms of these quantities the fibre can be re-expressed as

$$
g_{f}=\left(1+\frac{R^{2} r^{2}}{U}\right)^{-2} \frac{d R^{2}}{U^{2}}+\frac{R^{2}}{4} d t^{2} .
$$

In a singularity point $r_{0}$ we have that $V\left(r_{0}\right)=0$. Let us also suppose that $U\left(r_{0}\right)=p \in Z$. In this specific case the fibre metric (6.22) near the singularity takes the form

$$
g_{f} \simeq \frac{1}{p^{2}}\left(d R^{2}+R^{2} d \tau^{2}\right),
$$

where $2 p \tau=t$. This means that the fibre metric extends smoothly across the singularity $R=0$. The question now is to find values of the parameters $\Lambda$ and $a$ such that the conditions $U\left(r_{0}\right)=p \in Z$ and $V\left(r_{0}\right)=0$ are realized. By using the expressions (6.21) for $U$ and $V$ it is found that these conditions reduce to an algebraic equation for $\Lambda$ and $a$ with solution

$$
\Lambda=\frac{2(n+1-p)}{r_{0}^{2}}, \quad a^{2 n+2}=\left(r_{0}\right)^{2 n+2} \frac{p+1}{n+2},
$$

which gives a further relation

$$
\left(a^{2} \Lambda\right)^{n+1}=(2 n+2-2 p)^{n+1} \frac{p+1}{n+2} .
$$

Thus, the metric extends smoothly across the singularity only if the parameters $a$ and $\Lambda$ are related by (6.24). We see from (6.23) that if $\Lambda<0$ then $p \geq n+2$. Also

$$
\frac{d V}{d r}=\frac{2 n+2}{r^{2 n+3}} a^{2 n+2}-\frac{\Lambda}{n+2} r>0
$$

for $r>0$. This means that the metric is non-singular for $r>r_{0}$. In particular if the Fubbini-Study metric is used as the base space, then the desingularization procedure presented before corresponds to the desingularization $O(-p) \rightarrow C^{n+1} / Z_{p}, O(-(n+1))$ being the canonical bundle of $P^{n}[66]$.

Also, the case $\Lambda<0$ corresponds to the parameters $p \geq 0$ and $s \leq 0$ in (6.15). The fibre metric $g_{f}$ is two-dimensional and by Gauss theorem, it is conformally flat. This means that there exists a co-ordinate system $(\rho, \tau)$ 
such that

$$
p^{2} g_{f}=p^{2} w d z^{2}+\frac{4 d \tau^{2}}{w}=\Omega^{2}(\rho)\left(d \rho^{2}+\rho^{2} d \tau^{2}\right),
$$

$\Omega^{2}$ being a conformal factor. From this equality we get the relation

$$
\frac{d \rho}{d z}=p \frac{w^{1 / 2}}{\Omega}, \quad \frac{4}{w}=\rho^{2} \Omega^{2} .
$$

By differentiating the second one we get

$$
4 \frac{d w^{-1}}{d z}=2 \rho^{2} \Omega \frac{d \Omega}{d z}+2 \rho \Omega^{2} \frac{d \rho}{d z}=2 \Omega \rho^{2} \frac{d \Omega}{d z}+2 p \Omega^{2} \rho \frac{w^{1 / 2}}{\Omega} .
$$

By introducing the first relation one obtains

$$
4 \frac{d w^{-1}}{d z}=\frac{2}{w \Omega} \frac{d \Omega}{d z}+p \Longleftrightarrow \frac{d \log \left(w \Omega^{2}\right)}{d z}=-p w .
$$

From the last equation we obtain

$$
\Omega^{2} w=C \exp \left\{-p \int_{0}^{z} w d z\right\}
$$

and this, together with the second (6.25) yields

$$
\rho^{2}=\frac{4}{w \Omega^{2}}=4 C^{-1} \exp \left\{p \int_{0}^{z} w d z\right\} .
$$

From (6.15) it is obtained that

$$
w^{-1}=p z^{2}+q+\frac{s}{z^{n}}
$$

and using that for $\Lambda \leq 0$ we have $p \geq 0$ and $s \leq 0$; it follows that for any positive constant $C_{0}$ there exists a value $z_{0}>\sqrt{r_{0}}$ such that any $z>z_{0}$ we have that

$$
w^{-1} \geq \frac{p}{n+2} z^{2}+C_{0}^{2},
$$

from where it follows that

$$
\int_{\sqrt{r_{0}}}^{z} w \leq \int_{\sqrt{r_{0}}}^{z_{0}} w+\frac{A_{0}}{C_{0}^{2}}\left(\arctan \frac{z}{A_{0}}-\arctan \frac{z_{0}}{A_{0}}\right), \quad A_{0}=C_{0} \sqrt{\frac{n+2}{A}} .
$$

From the last inequality it is seen that for $z \rightarrow \infty$ the function $\rho$ approaches to a constant. In other words $\rho$ is a bounded function and hence the fibre metrics are defined on an open disk. This result is independent on the choice of the base space and is one of the key ingredient to prove that the open disk bundle of $O(-p) \rightarrow P^{n}$ admits a complete Kahler-Einstein metric with negative scalar curvature with $S U(n+1) \times U(1)$ invariant [66]. 


\subsection{The Calabi-Yau limit}

It is of interest to consider the Ricci flat limit of the metrics defined by (6.19) and (6.20). The resulting metric will be Ricci flat Kahler, thus Calabi-Yau, and its holonomy will be included in $S U(3)$. But we have already mentioned that these metrics are complete only if the parameters are constrained by (6.23). In the Ricci flat limit this condition is not satisfied and thus we do not have criteria to know if the result will be a complete metric, except in the case $a=0$ for which $V=1$. We see that it deserves the attention to study the Ricci flat limit $A=-2 \Lambda=0$ of equations (6.12) directly, instead of taking the limit to known solutions.

In references $[68,69]$ there have been made certain advances in constructing complete Calabi-Yau metrics, which we describe here briefly. The Ricci flat limit of the system $(6.12)$ is

$$
\begin{gathered}
4 \frac{\partial^{2} u}{\partial \xi_{\lambda} \partial \bar{\xi}_{\mu}}+B \frac{\partial h_{\lambda \mu}}{\partial z}=0 \\
\frac{\partial u}{\partial z}=w \\
4 \frac{\partial^{2} h_{a b}}{\partial z^{2}}+\frac{\partial^{2} w}{\partial \xi^{a} \partial \bar{\xi}^{b}}=0
\end{gathered}
$$

From (6.10) it is obtained that $u=\log w^{-1} \operatorname{det} h$. From this equality together with the second equation (6.26) it is deduced that

$$
w^{-1}=\frac{\int_{0}^{z} \operatorname{det} h}{\operatorname{det} h} .
$$

In addition, by multiplying the first of (6.26) by $d \xi_{\lambda} \wedge \xi_{\mu}$ and summing over the repeated indices gives

$$
\frac{d}{d z} \Omega_{h}(z)=-i \partial \bar{\partial} u
$$

Combining formula (6.28) with the definition of $u$ gives

$$
\frac{d}{d z} \Omega_{h}(z)=\rho(h)-i \partial \bar{\partial} \log w
$$

and from the last formula together with (6.27) we obtain

$$
\frac{d}{d z} \Omega_{h}(z)=-i \partial \bar{\partial} \int_{0}^{z} \operatorname{det} h .
$$

If we were able to find a triplet $\left(h(z), \Omega_{h}(z), w\right)$ solving these equations then we will construct a Calabi-Yau metric in six dimensions with local form

$$
g=h+w d z^{2}+w^{-1}(d \tau+A)^{2},
$$


$A$ being a one-form obtained from (6.7), which in our case reduces to

$$
d A=\frac{i}{2} \partial_{z} h_{a b} d \xi^{a} \wedge d \bar{\xi}^{b}+i \frac{\partial w}{\partial \xi^{k}} d z \wedge d \xi^{k}-i \frac{\partial w}{\partial \bar{\xi}^{k}} d z \wedge d \bar{\xi}^{k} .
$$

Here $\left(\xi^{k}, \bar{\xi}^{k}\right)$ are complex co-ordinates for $h(z)$.

A simple solution can be found starting with a four-dimensional KahlerEinstein metric $g_{4}$ with Kahler form $K$ defined over a manifold $X_{4}$, as in the previous subsection. Let us consider the two-form

$$
\Omega_{h}(z)=\bar{J}+z \rho(\bar{J}),
$$

$\bar{J}$ being the Kahler form for $g_{4}$. Because $g_{4}$ is Kahler-Einstein we have that $\rho(\bar{J})=\Lambda \bar{J}$. This implies that

$$
\Omega_{h}(z)=(1+\Lambda z) \bar{J} .
$$

We also have that

$$
\Omega_{h}(z) \wedge \Omega_{h}(z)=P(z) \bar{J} \wedge \bar{J}
$$

where $P(z)=(1+\Lambda z)^{2}$ and therefore $\rho(\bar{J})=\rho\left(\Omega_{h}\right)$. By introducing (6.32) in (6.29) and using $\rho(\bar{J})=\rho\left(\Omega_{h}\right)$ we see that $\Omega_{h}(z)$ is a solution of (6.29). The corresponding metric $h(z)$ is simply a $z$-dependent dilatation of $g_{4}$, namely

$$
h(z)=(1+\Lambda z) g_{4},
$$

and from (6.27) we see that

$$
w^{-1}=\frac{\int_{0}^{z}(1+\Lambda x)^{2} d x}{(1+\Lambda z)^{2}}=\frac{(1+\Lambda z)^{3}-1}{3 \Lambda(1+\Lambda z)^{2}} .
$$

By defining $r^{2}=1+\Lambda z$ the metric takes the following asymptotically conical form

$$
g_{6}=\frac{r^{2}}{9}\left(1-\frac{1}{r^{6}}\right)(d \tau+A)^{2}+\frac{r^{6}}{\left(r^{6}-1\right)} d r^{2}+\frac{\Lambda}{3} r^{2} g_{4}
$$

with $d A=\bar{J}$. This metric possesses holonomy in $S U(3)$ and depends on $\Lambda$ and the other parameters of the basis $g_{4}$. An important result given in [68] is that if $\Lambda>0$ then the metric (6.35) is complete over the canonical bundle $K_{X}$ of $X_{4}$. In addition it is clear that (6.35) is asymptotically conical, i.e., for large values of $r$ it tends to a cone of the form $d r^{2}+r^{2} g_{5}, g_{5}$ being the Einstein-Sasaki metric given by

$$
g_{5}=\frac{1}{9}(d t+A)^{2}+\frac{\Lambda}{3} g_{4} .
$$

Nevertheless this metric is Calabi-Yau for any value of $r$, not only asymptotically, and thus (6.35) provides a deformation of such cones without spoiling the Calabi-Yau condition. 
In order to illustrate this construction, we can extend the Kahler-Einstein metrics obtained in [40-44] to an asymptotically conical Calabi-Yau metric. The result is

$$
\begin{aligned}
g_{6}= & \frac{r^{6}}{\left(r^{6}-1\right)} d r^{2}+\frac{r^{2}}{9}\left(1-\frac{1}{r^{6}}\right)(d \tau-\cos \theta d \varphi+y(d \beta+c \cos \theta d \varphi))^{2} \\
& +2 r^{2} \frac{(1-c y)}{6}\left(d \theta^{2}+\sin ^{2} \theta d \varphi^{2}\right) \\
& +\frac{2 r^{2} d y^{2}}{w(y) q(y)}+\frac{2 w(y) q(y)}{36}(d \beta+c \cos \theta d \varphi)^{2}
\end{aligned}
$$

where we have defined

$$
w(y)=\frac{2\left(a-y^{2}\right)}{1-c y}, \quad q(y)=\frac{a-3 y^{2}+2 c y^{3}}{a-y^{2}} .
$$

If we select $c=0$ and $a=3$ the metric will be asymptotically a cone over $T^{1,1}$. If we select instead $c=a=1$ then the five-dimensional metric will be $S^{5}$.

There exist other solutions $\left(h(z), \Omega_{h}(z), w\right)$ of the system $(6.27)$ to $(6.29)$ that can be found starting with a Kahler manifold $M$ with metric $g_{4}$ which is not Einstein, but possess constant eigenvalues of the Ricci curvature. Let us consider first the case in which the metric possesses two different eigenvalues $\lambda_{1}$ and $\lambda_{2}$ with multiplicity two. This case has been studied recently in [72]. We will show that (6.32) still represents a solution although in this case $\rho(\bar{J}) \neq \Lambda \bar{J}$. For any Kahler manifold $M$ with constant eigenvalues of the Ricci curvature the Ricci form $\rho$ and the Kahler form $\bar{J}$ will be generically

$$
\bar{J}=\bar{J}_{1}+\bar{J}_{2}, \quad \rho=\lambda_{1} \bar{J}_{1}+\lambda_{2} \bar{J}_{2} .
$$

In addition we always have that

$$
\rho \wedge \bar{J}=s \omega
$$

$\omega$ being the volume form of $M$ and $s$ the scalar curvature, which in our case is $s=2 \lambda_{1}+2 \lambda_{2}$. We also have that

$$
\bar{J}_{1} \wedge \bar{J}_{1}=\bar{J}_{2} \wedge \bar{J}_{2}=0, \quad \bar{J}_{1} \wedge \bar{J}_{2}=2 \omega .
$$

By using this relation it is direct to check that

$$
\Omega_{h}(z) \wedge \Omega_{h}(z)=P(z) \bar{J} \wedge \bar{J}
$$

where $P(z)=\left(1+\lambda_{1} z\right)\left(1+\lambda_{2} z\right)$. Therefore we have again that $\rho(\bar{J})=$ $\rho\left(\Omega_{h}\right)$ and thus (6.32) is a solution of (6.29). The function $w$ is given by 
(6.27), the result is

$$
\begin{aligned}
w^{-1}= & \frac{\int_{0}^{z} P(x) d x}{P(z)}=\frac{1+\lambda_{1} z}{2 \lambda_{1}}+\frac{1+\lambda_{2} z}{2 \lambda_{2}} \\
& -\frac{1}{\left(1+\lambda_{1} z\right)\left(1+\lambda_{2} z\right)}\left(\frac{1}{2 \lambda_{1}}+\frac{1}{2 \lambda_{2}}\right) .
\end{aligned}
$$

The metric $h(z)$ is the metric for which $\Omega_{h}(z)$ is the Kahler form. The procedure in order to find it is as follows. One needs to find a basis of soldering forms $\widetilde{e}^{i}(z)$ such that

$$
\Omega_{h}(z)=\widetilde{e}^{1}(z) \wedge \widetilde{e}^{2}(z)+\widetilde{e}^{3}(z) \wedge \widetilde{e}^{4}(z) .
$$

The metric $h(z)$ will be given by $h(z)=\widetilde{e}^{i}(z) \otimes \widetilde{e}^{i}(z)$.

A special case of Kahler spaces with two degenerate eigenvalues is given as follows. The two forms $\bar{J}_{i}$ are characterized by

$$
\bar{J}_{1}=\bar{J}\left(\pi_{1} X, \pi_{1} Y\right), \quad \bar{J}_{2}=\bar{J}-\bar{J}_{1}
$$

$\pi_{1}$ being the projection from $T M$ to $E_{1}, E_{1}$ being the corresponding $J$ invariant subspace associated to the eigenvalue $\lambda_{1}$. The closure of $\bar{J}$ and $\rho$ implies that $\bar{J}_{i}$ are also closed. The almost complex structure $\widetilde{J}$ defined by $\left.\widetilde{J}\right|_{E_{1}}=\left.J\right|_{E_{1}}$ and $\left.\widetilde{J}\right|_{E_{2}}=-\left.J\right|_{E_{2}}$ commutes with $J$ and the corresponding two-form

$$
\overline{\widetilde{J}}=\bar{J}_{1}-\bar{J}_{2}
$$

is sympletic and possesses opposite orientation with respect to the one defined by $\bar{J}$. This means that

$$
\overline{\widetilde{J}} \wedge \overline{\widetilde{J}}=-\bar{J} \wedge \bar{J}=2 \omega
$$

It has been shown that the sympletic two-form $\widetilde{J}$ is integrable if and only if the base space $M$ is a direct product of two Kahler-Einstein spaces [72]. In this case $\left(g_{4}, \widetilde{J}\right)$ will be a Kahler structure with orientation opposite to $\left(g_{4}, J\right)$, and $\bar{J}_{1}$ and $\bar{J}_{2}$ will be the Kahler forms for such metrics. As an example we can consider the product of the two-dimensional FubbiniStudy metric $g_{f s}$ with the Bergmann one $g_{b}$. We normalize the curvature as $\lambda= \pm 1$. With the corresponding Kahler forms $\bar{J}_{f s}$ and $\bar{J}_{b}$ we consider the 
two-form

$$
\Omega_{h(z)}=(1+z) \bar{J}_{1}+(1-z) \bar{J}_{2},
$$

which by construction is a solution of (6.27). The corresponding metric $h(z)$ is given by

$$
h(z)=(1+z) g_{f s}+(1-z) g_{b}
$$

From (6.37) we see that $w=1 / z$ in this case, and the Calabi-Yau metric reads

$$
g_{6}=(1+z) g_{f s}+(1-z) g_{b}+\frac{1}{z} d z^{2}+z(d \tau+A)^{2}
$$

$A$ being given by $d A=\bar{J}_{1}-\bar{J}_{2}$. Observe that in general $d A=\rho(\bar{J})$.

Another class of Kahler manifolds with constant Ricci eigenvalues are homogeneous Kahler manifolds, for which the holomorphic isometries act transitively. There also exist non-homogeneous Kahler metrics in the literature with constant eigenvalues of the Ricci curvature. An example is the family

$$
g=e^{u} x\left(d x^{2}+d y^{2}\right)+x d z^{2}+\frac{1}{x}(d t+y d z)^{2},
$$

which possesses this property if $u$ is a function that satisfies

$$
u_{x x}+u_{y y}=s x e^{u} .
$$

The constant $s$ is the scalar curvature of the metric. The family (6.39) is Kahler and in general non-homogeneous, except for certain subcases. For instance by selecting $u=3 \log x$ the Kahler metric is obtained

$$
g=\frac{d x^{2}}{x^{2}}+\frac{d y^{2}}{x^{2}}+x d z^{2}+\frac{(d t+y d z)^{2}}{x^{2}}
$$

with Kahler form

$$
\bar{J}=-d z^{2} \wedge d y+d y \wedge d\left(\frac{1}{x}\right) .
$$

This metric possesses two different eigenvalues of the curvature tensor. If we make the variable change

$$
u_{1}=\frac{x^{2}+y^{2}-1}{2 x}, \quad v_{1}=-\frac{y}{x}, \quad u_{2}=t, \quad v_{2}=z
$$

then the metric takes the form

$$
\begin{aligned}
g= & \left(-u_{1}+\sqrt{u_{1}^{2}+v_{1}^{2}+1}\right) d u_{2}^{2}+\left(u_{1}+\sqrt{u_{1}^{2}+v_{1}^{2}+1}\right) d v_{2}^{2} \\
& -2 v_{1} d u_{2} d v_{2} \frac{1}{\left(u_{1}^{2}+v_{1}^{2}+1\right)}\left[\left(1+v_{1}^{2}\right) d u_{1}^{2}+\left(1+u_{1}^{2}\right) d v_{1}^{2}-2 u_{1} v_{1} d u_{1} d v_{1}\right]
\end{aligned}
$$

It has been shown that this metric is homogeneous and non-symmetric in [73] and the Ricci eigenvalues are $\left(0,0,-\frac{3}{8},-\frac{3}{8}\right)$. But the metrics (6.39) are non-homogeneous in general. 
It has been shown that in general, the resulting Calabi-Yau metric will be complete if the Ricci eigenvalues are all positive [68]. This is not the case for many of the examples that we have constructed so we cannot decide whether or not the resulting Calabi-Yau metrics are complete. It is then of interest to classify which solutions of the equation (6.39) give rise to metrics with positive eigenvalues. Nevertheless this could be a hard task, due to the non-linear nature of (6.39).

\section{Discussion}

Along this paper we considered an infinite family of tri-Sasaki seven-metrics and its squashed version, which are of weak $G_{2}$ holonomy. We have found in particular, a large class of examples with $T^{3}$ isometry. We constructed several new supergravity backgrounds and their deformation by use of the Maldacena-Lunin prescription. This should correspond to a marginal deformation in the dual theory. We have found in certain manifold limit a rotating configuration reproducing the logarithmic behaviour of the difference between the spin and the energy. We have found the same behaviour for the deformed background, although this is not a direct product of $A d S_{4}$ with a seven-dimensional space.

We want to emphasize that there is an underlying linear structure describing all the backgrounds presented along this work. This is given by (3.29) and in fact, all the spaces that we have presented here are completely determined in terms of solutions of this equation. It will be nice to make a more deep analysis of the dual conformal theories of these backgrounds. Notice that the complete examples that we have presented are defined in terms of certain twistors. This is in part, a consequence of the underlying linear structure. It will be interesting to understand how these twistors are realized in the dual conformal field theory. Perhaps the methods presented in [61] could be useful for this purpose. Another interesting task is to figure out the $p p$ wave limit of the metrics we presented and to obtain realization of the holographic renormalization group in the lines described in [91]. We will return with this subject in a future investigation.

\section{Appendix A: Quaternionic Kahler spaces in dimension higher than four}

The generators $J^{i}$ of the Lie algebra $s p(1)$ of $S p(1) \simeq S U(2)$ have the multiplication rule

$$
J^{i} \cdot J^{j}=-\delta^{i j} I+\epsilon_{i j k} J^{k},
$$


which implies the $s o(3) \simeq s u(2)$ commutation rule

$$
\left[J^{i}, J^{j}\right]=\epsilon_{i j k} J^{k} .
$$

We see that $J^{i} J^{i}=-I$ and therefore $J^{i}$ will be called almost complex structures. An useful $4 n \times 4 n$ representation is

$$
\begin{aligned}
J^{1} & =\left(\begin{array}{cccc}
0 & -I_{n \times n} & 0 & 0 \\
I_{n \times n} & 0 & 0 & 0 \\
0 & 0 & 0 & -I_{n \times n} \\
0 & 0 & I_{n \times n} & 0
\end{array}\right), \\
J^{2} & =\left(\begin{array}{cccc}
0 & 0 & -I_{n \times n} & 0 \\
0 & 0 & 0 & I_{n \times n} \\
I_{n \times n} & 0 & 0 & 0 \\
0 & -I_{n \times n} & 0 & 0
\end{array}\right) \\
J^{3}=J^{1} J^{2} & =\left(\begin{array}{cccc}
0 & 0 & 0 & -I_{n \times n} \\
0 & 0 & -I_{n \times n} & 0 \\
0 & I_{n \times n} & 0 & 0 \\
I_{n \times n} & 0 & 0 & 0
\end{array}\right) .
\end{aligned}
$$

The group $S O(4 n)$ is a Lie group and this means in particular that for any $S O(4 n)$ tensor $A_{b}^{a}$ the commutator $\left[A, J^{i}\right]$ will take also values in $S O(4 n)$. We will say that $A$ belongs to the subgroup $S p(n)$ of $S O(4 n)$ if and only if

$$
\left[A, J^{i}\right]=0 .
$$

Condition (A:.4) together with (A:.2) implies that a tensor $B_{b}^{a}$ belongs to the subgroup $S p(n) \times S p(1)$ if and only if

$$
\left[B, J^{i}\right]=\epsilon_{i j k} J^{j} B_{-}^{k},
$$

$B_{-}^{k}$ being the component of $B$ in the basis $J^{k}$. Both conditions are independent of the representation.

We will write a metric over a $4 n$-dimensional manifold $M$ as $g=\delta_{a b} e^{a} \otimes$ $e^{b}, e^{a}$ being the $4 n$-basis for which $g$ is diagonal (or vielbein basis). Let us define the triplet of $(1,1)$ tensors

$$
J^{i}=\left(J^{i}\right)_{b}^{a} e_{a} \otimes e^{b},
$$

defined by the matrices (A:.3). If the holonomy is in $S p(n) \times S p(1)$, then from the beginning $\omega_{b}^{a}$ will take values on its Lie algebra $s p(n) \oplus s p(1)$. As 
we saw before, this implies that

$$
\left[\omega, J^{i}\right]=\epsilon_{i j k} J^{j} A^{k} .
$$

As usual, the connection $\omega_{b}^{a}$ is defined through

$$
\nabla_{X} e^{a}=-\omega_{b}^{a}(X) e^{b}
$$

together with the Levi-Civita conditions $\nabla g=0$ and $T(X, Y)=0$. Using the chain rule $\nabla(A \otimes B)=(\nabla A) \otimes B+A \otimes(\nabla B)$ for tensorial products show us that in the vielbein basis

$$
\left[\omega, J^{i}\right]=\nabla_{X} J^{i}
$$

Comparing (A:.6) and (A:.7) we see that quaternionic Kahler manifold is defined by the relation

$$
\nabla_{X} J^{i}=\epsilon_{i j k} J^{j} A^{k}
$$

which is independent on the election of the frame $e^{a}$. This proves that (2.9) describes quaternion Kahler metrics [28] in dimension higher than four.

The basis $e^{a}$ for a metric $g$ is defined up to an $S O(4 n)$ rotation. Under this $S O(4 n)$ transformation the tensors (A:.5) are also transformed, but it can be shown that the multiplication (A:.1) is unaffected. In other words, given the tensors $J^{i}$ one can construct a new set of complex structures

$$
J^{i}=C_{j}^{i} J^{j}, \quad J^{\prime i} \cdot J^{\prime j}=-\delta^{i j} I+\epsilon_{i j k} J^{\prime k} \Longleftrightarrow C_{k}^{i} C_{j}^{k}=\delta_{j}^{i}
$$

This can be paraphrased by saying that a quaternionic Kahler manifold has a bundle $V$ of complex structures parameterized by the sphere $S^{2}$. Using the textbook properties of $\nabla$ it can be seen that (2.9) is unaltered under such rotations.

Let us define three new tensors $\left(\bar{J}^{i}\right)_{a b}$ by $\left(\bar{J}^{i}\right)_{a b}=\left(J^{i}\right)_{a}^{c} \delta_{c b}$. From (A:.3) it follows that

$$
\left(J^{i}\right)_{b}^{a}=-\left(J^{i}\right)_{a}^{b} \Longleftrightarrow\left(\bar{J}^{i}\right)_{a b}=-\left(\bar{J}^{i}\right)_{b a} .
$$

This show that $\left(\bar{J}^{i}\right)_{a b}$ are the components of the two-forms $\bar{J}^{i}$ defined by

$$
\bar{J}^{i}=\left(\bar{J}^{i}\right)_{a b} e^{a} \wedge e^{b} .
$$

The forms (A:.9) are known as the hyperKahler forms. From (2.9) it is obtained that

$$
\nabla_{X} J^{i}=\epsilon_{i j k} J^{j} A^{k} \Longrightarrow d \bar{J}^{i}=\epsilon_{i j k} A^{j} \wedge \bar{J}^{k}
$$

$d$ being the usual exterior derivative. The last implication proves the relation (2.10).

If we change the frame $e^{a}$ to a new one $x_{\mu}$ then the definition $\left(\bar{J}^{i}\right)_{a b}=$ $\left(J^{i}\right)_{a}^{c} \delta_{c b}$ should be modified by the covariant $\left(\bar{J}^{i}\right)_{\alpha \beta}=\left(J^{i}\right)_{\alpha}^{\gamma} g_{\gamma \beta}$. Here the 
greek index indicates the components in the new basis and $g_{\gamma \beta}$ are the corresponding components of the metric. Therefore

$$
\left(\bar{J}^{i}\right)_{a b}=-\left(\bar{J}^{i}\right)_{b a} \Longleftrightarrow\left(J^{i}\right)_{\alpha}^{\gamma} g_{\gamma \beta}=\left(J^{i}\right)_{\beta}^{\gamma} g_{\gamma \alpha} .
$$

The last relation is equivalent to

$$
g\left(J^{i} X, Y\right)=g\left(X, J^{i} Y\right) \Longleftrightarrow g(X, Y)=g\left(J^{i} X, J^{i} Y\right)
$$

for arbitrary vector fields $X$ and $Y$ in $T M$. Then the metric $g$ will be always quaternion Hermitian with respect to the complex structures. Relation (2.8) is also invariant under the automorphism of the complex structures.

In general, if in a given manifold there exist three complex structures satisfying (A:.1), and we take intersecting co-ordinate neighbourhoods $U$ and $U^{\prime}$, then we have two associated bases $J^{i}$ and $J^{\prime i}$. Both bases should be related by an $S O(3)$ transformation in order to satisfy (A:1). This means that any quaternion Kahler space is orientable [28]. Consider now the fundamental four-form

$$
\Theta=\bar{J}^{1} \wedge \bar{J}^{1}+\bar{J}^{2} \wedge \bar{J}^{2}+\bar{J}^{3} \wedge \bar{J}^{3},
$$

and the globally defined $(2,2)$ tensor

$$
\Xi=J^{1} \otimes J^{1}+J^{2} \otimes J^{2}+J^{3} \otimes J^{3} .
$$

By means of the formula $(\mathrm{A}: 8)$ it follows that both tensors $(\mathrm{A}: \mathrm{10})$ and (A:.11) are globally defined on the manifold $M$. For a quaternionic Kahler manifold it is obtained directly from (2.9) and (2.10) that [28]

$$
\nabla \Theta=0, \quad \nabla \Xi=0 .
$$

In $D=8$ for a quaternion Kahler manifold $d \Theta=0$ and if the manifold is of dimension at least 12 then $d \Theta$ determines $\nabla \Theta$ completely. In particular $d \Theta=0$ implies $\nabla \Theta=0[79]$.

One of the most important consequences of (2.9) is that quaternionic Kahler spaces are always Einstein with cosmological constant [26]. The proof is briefly as follows. From the definition of the curvature tensor $R(X, Y)=$ $\left[\nabla_{X}, \nabla_{Y}\right]-\nabla_{[X, Y]}$ together with (2.9) it follows in the vielbein basis that

$$
R_{i j m}^{l}\left(J^{a}\right)_{k}^{m}-R_{i j k}^{m}\left(J^{a}\right)_{m}^{l}=\epsilon_{a b c}\left(F^{b}\right)_{i j}\left(J^{c}\right)_{k}^{l}
$$

where $R_{i j m}^{l}$ are the components of the curvature tensor and the two-form $F^{a}$ was defined as ${ }^{3}$

$$
F^{a}=d \omega_{-}^{a}+\epsilon_{a b c} \omega_{-}^{b} \wedge \omega_{-}^{c} .
$$

\footnotetext{
${ }^{3}$ In the physical literature sometimes the three components $\omega_{-}$are referred to as an $S U(2)$ vector potential and $F^{a}$ as the corresponding strength tensor.
} 
We can rewrite (A:.12) as a commutator

$$
\left[R(X, Y), J^{a}\right]=\epsilon_{a b c} F^{b} J^{c},
$$

$X$ and $Y$ being arbitrary vector fields. Multiplying (A:.12) by $\left(J^{a}\right)_{l}^{s}$ and contracting indices, and then multiplying by $\left(J^{b}\right)_{l}^{k}$ and using the identity ${ }^{4}$

$$
\left(J^{a}\right)_{l}^{s}\left(J^{b}\right)_{s}^{l}=4 n \delta^{a b},
$$

gives the formula

$$
F_{i j}^{a}=\frac{1}{2 n} R_{i j k}^{l}\left(J^{a}\right)_{l}^{k} .
$$

Inserting (A:.13) into (A:.12) yields

$$
R_{i j k}^{l}\left(J^{a}\right)_{l}^{k}=\frac{2 n}{2+n} R_{i m}\left(J^{a}\right)_{j}^{m},
$$

which can also be expressed as

$$
R_{-}^{i}=\frac{2 n}{2+n} R \bar{J}^{i},
$$

$R$ being the scalar curvature and $R_{-}^{i}$ the $S p(1)$ components of the curvature tensor. The second Bianchi identities together with (A:14) shows that $R$ is constant and thus $R_{i j} \sim g_{i j}[26]$. Thus, in any dimension, quaternionic Kahler spaces are always Einstein with non-zero cosmological constant $\lambda$.

Because $R$ is a constant we see from (A:.14) that

$$
R_{-}^{i}=\Lambda \bar{J}^{i},
$$

$\Lambda$ being a certain constant. We also have from (A:.13) that

$$
F^{i}=\Lambda^{\prime} \bar{J}^{i},
$$

$\Lambda^{\prime}$ being another constant. In the limit $\lambda \rightarrow 0$ the constants $\Lambda$ and $\Lambda^{\prime}$ go simultaneously to zero.

If there exists a rotation of the local frame for which $\omega_{-}=0$ then the complex structures are locally covariantly constant, i.e.,

$$
\nabla_{X} J^{i}=0 .
$$

In this case $R_{-}^{i}=F^{i}=0$ thus the space has self-dual curvature, which implies Ricci flatness. This space is called hyperKahler, and (A:.17) shows

\footnotetext{
${ }^{4}$ This is clearly true in the representation (A:.3).
} 
that they are Kahler with respect to any of the complex structures. Condition (A:.17) implies that the holonomy is in $S p(n)$ and that

$$
d \bar{J}^{a}=0
$$

together with the annulation of the Niejenhuis tensor given by

$$
N(X, Y)=[X, Y]+J[X, J Y]+J[J X, Y]-[J X, J Y] .
$$

A complex structure for which $N(X, Y)=0$ is called integrable.

\section{Appendix B: Quaternion Kahler manifolds in dimension four}

As we saw starting from the previous section, in four dimensions the statement that the holonomy is $\Gamma \subseteq S p(n) \times S p(1)$ is trivial due to the isomorphism $S O(4) \simeq S U(2)_{L} \times S U(2)_{R} \simeq S p(1) \times S p(1)$. We will modify this definition and we will say that a four-dimensional manifold $M$ is quaternionic Kahler if (A:.15) holds. This condition is not trivial, we will show next that quaternion Kahler spaces in $d=4$ are Einstein (as in the higher dimensional case) and with self-dual Weyl tensor.

Let us consider a four-dimensional metric $g=\delta_{a b} e^{a} \otimes e^{b}$ and the connection $\omega_{b}^{a}$ given by the first Cartan equation

$$
d e^{a}+\omega_{b}^{a} \wedge e^{b}=0, \quad \omega_{i b}^{a}=-\omega_{b i}^{a} .
$$

The notation $S U(2)_{ \pm}$denotes the $S U(2)_{L}$ and $S U(2)_{R}$ groups, respectively. The $S U(2)_{ \pm}$components of the spin connection are explicitly

$$
\omega_{ \pm}^{a}=\omega_{0}^{a} \pm \epsilon_{a b c} \omega_{c}^{b} .
$$

The curvature tensor is given by the second Cartan equation

$$
R_{b}^{a}=d \omega_{b}^{a}+\omega_{s}^{a} \wedge \omega_{b}^{s}=R_{b, s t}^{a} e^{s} \wedge e^{t}
$$

and the $S U(2)$ parts are

$$
R_{ \pm}^{a}=R_{0}^{a} \pm \epsilon_{a b c} R_{c}^{b} .
$$

The Ricci tensor is defined in the diagonal basis by $R_{i j}=R_{i, a j}^{a}$ and the scalar curvature is $R_{i i}=R$.

Instead of using the basis $e^{a} \wedge e^{b}$ we can use the basis $\bar{J}_{ \pm}^{a}=e^{0} \wedge e^{a} \pm$ $\epsilon_{a b c} e^{b} \wedge e^{c}$. Then it follows that $\bar{J}_{ \pm}^{a}$ are separately complex structures with 
definite self-duality properties, i.e.,

$$
* \bar{J}_{ \pm}^{a}= \pm \bar{J}_{ \pm}^{a} .
$$

In this basis

$$
R_{+}^{a}=A_{a b} \bar{J}_{+}^{b}+B_{a b} \bar{J}_{-}^{b}, \quad R_{-}^{a}=B_{a b}^{t} \bar{J}_{+}^{b}+C_{a b} \bar{J}_{-}^{b}
$$

where the matrices $A$ and $C$ are symmetric. The components of the Ricci tensor are

$$
\begin{aligned}
& R_{00}=\operatorname{Tr}(A+B), \quad R_{0 a}=\frac{\epsilon_{a b c}}{2}\left(B_{b c}^{t}-B_{b c}\right), \\
& R_{a b}=\operatorname{Tr}(A-B) \delta_{a b}+B_{a b}+B_{a b}^{t},
\end{aligned}
$$

and the scalar curvature is

$$
R=4 \operatorname{Tr}(A)=4 \operatorname{Tr}(C) .
$$

It is clearly seen from (B:.4) that the Einstein condition $R_{i j}=\Lambda \delta_{i j}$ is equivalent to $B=0$ and $\operatorname{Tr}(A)=\operatorname{Tr}(C)=\Lambda$.

The components of the Weyl tensor in the diagonal basis are given by

$$
W_{b c d}^{a}=R_{b c d}^{a}-\frac{1}{2}\left(\delta_{a c} R_{b d}-\delta_{a d} R_{b c}+\delta_{b d} R_{a c}-\delta_{b c} R_{a d}\right)+\frac{R}{6}\left(\delta_{a c} \delta_{b d}-\delta_{a d} \delta_{b c}\right) .
$$

The tensor $W$ is invariant under a conformal transformation $g \rightarrow \Omega^{2} g$ and the associated two-form is

$$
W_{b}^{a}=W_{b c d}^{a} e^{c} \wedge e^{d} .
$$

An explicit calculation shows that the $S U(2)_{ \pm}$of $W$ are

$$
\begin{aligned}
& W_{+}^{a}=W_{0}^{a}+\epsilon_{a b c} W_{c}^{b}=\left(A_{a b}-\frac{1}{3} \operatorname{Tr}(A) \delta_{a b}\right) \bar{J}_{+}^{b}, \\
& W_{-}^{a}=W_{0}^{a}-\epsilon_{a b c} W_{c}^{b}=\left(C_{a b}-\frac{1}{3} \operatorname{Tr}(C) \delta_{a b}\right) \bar{J}_{-}^{b} .
\end{aligned}
$$

From this expression we see that an Einstein space is self-dual (i.e., $W_{-}^{a}=0$ ) if it is equivalent to

$$
C_{a b}=\frac{\Lambda}{3} \delta_{a b} \Longleftrightarrow R_{-}^{a}=\frac{\Lambda}{3} \bar{J}_{-}^{a} .
$$

The second (B:.7) is the same as (A:.15) in four dimensions. Thus we conclude then that in $D=4$ quaternionic Kahler is the same as self-dual Einstein. 


\section{Appendix C: The solution-generating technique for IIB backgrounds}

The 11 supergravity backgrounds constructed in (5.13) possess three commuting Killing vectors. We can obtain $T^{2}$ IIA supergravity solutions by reduction along one isometry, say $\phi_{3}$. Also, by making a T-duality along another isometry, say $\phi_{1}$, we will obtain IIB supergravity backgrounds which are also toric. Now if we make the $S L(2, R)$ deformation of the original 11dimensional backgrounds and we make the IIB reduction we will obtain a new background, the IIB deformed one. Comparison between the resulting expression will give a technique in order to deform a IIB background into another one. The result will be a one-parameter deformation. This is a particular case of a two-parameter deformation that is known in the literature, which we will describe now.

Recall that any IIB background can be casted in the form

$$
\begin{aligned}
g_{I I B}= & F\left[\frac{1}{\sqrt{\Delta}}\left(D \alpha_{1}-C\left(D \phi_{2}\right)\right)^{2}+\sqrt{\Delta}\left(D \alpha_{2}\right)^{2}\right]+\frac{e^{2 \Phi / 3}}{F^{1 / 3}} \widetilde{g}, \\
B= & B_{12}\left(D \phi_{1}\right) \wedge\left(D \phi_{2}\right)+D \phi_{1} \wedge B_{1}+D \phi_{2} \wedge B_{2}-\frac{1}{2} A_{m} \wedge B_{m}+\frac{1}{2} \tilde{b}, \\
C^{(2)}= & C_{12}\left(D \phi_{1}\right) \wedge\left(D \phi_{2}\right)+D \phi_{1} \wedge C_{1}+D \phi_{2} \wedge C_{2}-\frac{1}{2} A_{m} \wedge C_{m}+\frac{1}{2} \tilde{c}, \\
& \quad e^{2 \Phi}=e^{2 \phi}, \quad C^{(0)}=\chi, \\
C^{(4)}= & -\frac{1}{2}\left(\tilde{d}+B_{12} \tilde{c}-\epsilon^{m n} B_{m} \wedge C_{n}-B_{12} A_{m} \wedge C_{m}\right) \wedge D \phi_{1} \wedge D \phi_{2} \\
& +\frac{1}{6}\left[C+3\left(\tilde{b}+A_{1} \wedge B_{1}-A_{2} \wedge B_{2}\right) \wedge C_{(1)}\right] \wedge D \phi_{1}+d_{4}+\hat{d}_{3} \wedge D \phi_{2},
\end{aligned}
$$

where

$$
D \phi_{2}=d \phi_{2}+A_{2}, \quad D \phi_{1}=d \phi_{1}+A_{1} .
$$

The effect of the $S L(3, R)$ transformation over these backgrounds is the following. We have three objects which transform as vectors and tensors

$$
\begin{aligned}
V^{(1)} & =\left(\begin{array}{c}
-B_{2} \\
A_{1} \\
C_{2}
\end{array}\right), \quad V^{(2)}=\left(\begin{array}{c}
B_{1} \\
A_{2} \\
-C_{1}
\end{array}\right): V^{(i)} \longrightarrow\left(\Lambda^{T}\right)^{-1} V^{(i)} ; \\
W & =\left(\begin{array}{c}
\tilde{c} \\
\tilde{d} \\
\tilde{b}
\end{array}\right) \longrightarrow \Lambda W
\end{aligned}
$$


and one matrix

$$
\begin{aligned}
M & =g g^{T}, \\
g^{T} & =\left(\begin{array}{ccc}
e^{-\phi / 3} F^{-1 / 3} & 0 & 0 \\
0 & e^{-\phi / 3} F^{2 / 3} & 0 \\
0 & 0 & e^{2 \phi / 3} F^{-1 / 3}
\end{array}\right)\left(\begin{array}{ccc}
1 & B_{12} & 0 \\
0 & 1 & 0 \\
\chi & -C_{12}+\chi B_{12} & 1
\end{array}\right)
\end{aligned}
$$

with transformation law

$$
M \longrightarrow \Lambda M \Lambda^{T}
$$

The scalars $\Delta, C$ as well as the three-form $C_{\mu \nu \lambda}$ stay invariant under these $S L(3, R)$ transformations. From this expression one can read the generic transformation of any of the fields.

We will restrict ourselves with a matrix of the form

$$
\begin{aligned}
\Lambda & =\left(\begin{array}{lll}
1 & \gamma & 0 \\
0 & 1 & 0 \\
0 & \sigma & 1
\end{array}\right), \quad \Lambda^{T}=\left(\begin{array}{lll}
1 & 0 & 0 \\
\gamma & 1 & \sigma \\
0 & 0 & 1
\end{array}\right) \\
\Lambda^{-1} & =\left(\begin{array}{ccc}
1 & -\gamma & 0 \\
0 & 1 & 0 \\
0 & -\sigma & 1
\end{array}\right), \quad\left(\Lambda^{T}\right)^{-1}=\left(\begin{array}{ccc}
1 & 0 & 0 \\
-\gamma & 1 & -\sigma \\
0 & 0 & 1
\end{array}\right) .
\end{aligned}
$$

Then the transformed fields are

$$
A_{1}^{\prime}=A_{1}, \quad A_{2}^{\prime}=A_{2}-\sigma A_{3}, \quad A_{3}^{\prime}=A_{3}, \quad \widetilde{c}^{\prime}=\gamma \widetilde{d}, \quad \widetilde{d^{\prime}}=\widetilde{d}, \quad \widetilde{b}^{\prime}=\sigma \widetilde{d} .
$$

In addition, the transformation law (C:.3) implies that $g^{T}$ should transform as $g^{T} \rightarrow R g^{T} \Lambda^{T}, R$ being an $S O(3)$ transformation. The Euler angles of this rotation should be selected in order that the non-diagonal matrix in the expression for $g^{T}$ conserves its form, i.e., the components $(2,1),(1,3)$ and $(2,3)$ should be zero. We have that

$$
\begin{aligned}
g^{T} \Lambda^{T}= & \left(\begin{array}{ccc}
e^{-\phi / 3} F^{-1 / 3} & 0 & 0 \\
0 & e^{-\phi / 3} F^{2 / 3} & 0 \\
0 & 0 & e^{2 \phi / 3} F^{-1 / 3}
\end{array}\right) \\
& \times\left(\begin{array}{ccc}
1+\gamma B_{12} & B_{12} & \sigma B_{12} \\
\gamma & 1 & \sigma \\
\chi+\gamma\left(\chi B_{12}-C_{12}\right) & \chi B_{12}-C_{12} & 1+\sigma\left(\chi B_{12}-C_{12}\right)
\end{array}\right)
\end{aligned}
$$

which is not of the desired form. We have to multiply this expression for a rotation matrix $R\left(\alpha_{1}, \alpha_{2}, \alpha_{3}\right)$ and the condition that the components $(2,1)$, 
$(1,3)$ and $(2,3)$ vanish give the following system of equations:

$$
\begin{gathered}
\cot \alpha_{2} B_{12}=-\cos \alpha_{3} \\
\sin \alpha_{2}+\cos \alpha_{3} \cos \alpha_{2} B_{12}=-(1+\sigma G) \sin \alpha_{3}, \\
\left(\sin \alpha_{1} \cos \alpha_{2}+\cos \alpha_{3} \sin \alpha_{2} \cos \alpha_{1}\right)\left(1+\gamma B_{12}\right) \\
+\left(\sin \alpha_{1} \sin \alpha_{2}-\cos \alpha_{3} \cos \alpha_{2} \cos \alpha_{1}\right) \gamma \\
-(\chi+\gamma G) \cos \alpha_{1} \sin \alpha_{3}=0 .
\end{gathered}
$$

The first two equations involve only $\alpha_{2}$ and $\alpha_{3}$. The angle $\alpha_{1}$ is then defined by the third equation, which turns out to be

$$
\tan \alpha_{1}=-\frac{\cos \alpha_{3} \cos \alpha_{2} \gamma-\cos \alpha_{3} \sin \alpha_{2}\left(1+\gamma B_{12}\right)-(\chi+\gamma G) \sin \alpha_{3}}{\left(1+\gamma B_{12}\right) \cos \alpha_{2}+\gamma \sin \alpha_{2}} .
$$

The transformation of $F$ and $\phi$ is then obtained by requiring that for the nondiagonal matrix in $g^{T}$ the diagonal elements are $g_{i i}^{T}=1$. The transformed components were worked out for instance in [21], the result is

$$
\begin{aligned}
g_{11}^{T}= & \frac{e^{-\phi / 3} \kappa}{\mu}, \quad g_{12}^{T}=\frac{e^{5 / 3 \phi}}{\mu \kappa}\left(B_{12}+\gamma B_{12}^{2}-B_{12} C_{12} \sigma+F^{2}(\gamma-\chi \sigma)\right) \\
g_{2,2}^{T}= & \frac{\left(e^{2 \phi} F^{2}\right)^{1 / 3}}{\kappa}, \\
g_{32}^{T}= & \frac{e^{-\phi / 3}}{\mu}\left(B_{12} \chi e^{2 \phi}+C_{12}^{2} \sigma e^{2 \phi}+B_{12}^{2} \sigma\left(1+\chi^{2} e^{2 \phi}\right)\right. \\
& \left.+F^{2} \sigma-C_{12} e^{2 \phi}\left(1+2 B_{12} \chi \sigma\right)\right) \\
g_{31}^{T}= & \frac{e^{-\phi / 3}}{\mu}\left(-C_{12} \gamma e^{2 \phi}+C_{12}^{2} \gamma \sigma e^{2 \phi}+B_{12} \chi^{2} e^{2 \phi} \sigma\left(1+B_{12} \gamma\right)\right. \\
& \left.+\sigma\left(B_{12}+B_{12}^{2} \gamma+F^{2} \gamma\right)-\chi e^{2 \phi}\left(-1+C_{12} \sigma+B_{12} \gamma\left(2 C_{12} \sigma-1\right)\right)\right) \\
g_{3,3}^{T}= & \left(\frac{e^{-\phi}}{F}\right)^{1 / 3} \sqrt{\left(B_{12}^{2}+F^{2}\right) \sigma^{2}+e^{2 \phi}\left(1-C_{12} \sigma+B_{12} \chi \sigma\right)^{2}} \\
\mu= & F^{1 / 3} \sqrt{\left(B_{12}^{2}+F^{2}\right) \sigma^{2}+e^{2 \phi}\left(1-C_{12} \sigma+B_{12} \sigma \chi\right)^{2}}, \\
\kappa^{2}= & F^{2} \sigma^{2}+e^{2 \phi}\left(\left(B_{12} \gamma\right)^{2}-2 B_{12} \gamma\left(C_{12} \sigma-1\right)\right. \\
& \left.+\left(C_{12} \sigma-1\right)^{2}+F^{2}(\gamma-\sigma \chi)^{2}\right) .
\end{aligned}
$$

The transformed fields are then

$B_{12}^{\prime}=\frac{g_{12}^{T}}{g_{11}^{T}}, \quad e^{\phi^{\prime}}=\frac{g_{33}^{T}}{g_{11}^{T}}, \quad \chi^{\prime}=\left(\frac{g_{22}^{T} g_{11}^{T}}{g_{33}^{T}}\right)^{1 / 3} g_{31}^{T}, \quad C_{12}^{\prime}=\chi^{\prime} B_{12}^{\prime}-g_{32}^{T} g_{22}^{T} g_{11}^{T}$, 
and are completely determined in terms of the fields of the original IIB supergravity solution. The procedure is explicitly defined.

\section{Appendix D: IIB deformed superbackgrounds for the spherical case}

Let us consider now the IIA reduction of our $T^{3}$ supergravity backgrounds of Section 5. In order to perform the reduction we need to make the decomposition

$$
M_{a b} D \phi_{a} D \phi_{b}=e^{-2 \phi_{D} / 3} \widetilde{h}_{m n} D \phi_{m} D \phi_{n}+e^{4 \phi_{D} / 3}\left(D \phi_{3}+N_{m} D \phi_{m}\right)^{2},
$$

with $m, n=1,2$. The metric $\widetilde{h}$ should not be confused with the $h$ appearing in (5.15)! We find that

$$
\phi_{D}=\frac{3}{4} \log \left(M_{33}\right), \quad N_{m}=\frac{M_{3 m}}{M_{33}}, \quad \widetilde{h}_{m n}=\frac{M_{m n} M_{33}-M_{3 m} M_{3 n}}{\sqrt{M_{33}}}
$$

and is straightforward to find the IIA reduced background. By making a T-duality [101] to the resulting IIA background we obtain the IIB solution

$$
\begin{aligned}
g_{I I B}= & \frac{1}{h_{11}}\left[\frac{1}{\sqrt{\Delta}}\left(D \phi_{1}-C D \phi_{2}\right)^{2}+\sqrt{\Delta}\left(D \phi_{2}\right)^{2}\right]+e^{2 \phi / 3} \widetilde{g}, \\
B= & \frac{h_{12}}{h_{11}} D \phi_{1} \wedge D \phi_{2}-D \phi_{2} \wedge C_{(32)}+D \phi_{1} \wedge A_{1}-\frac{1}{2} C_{(3)}+C_{(31)} \wedge A_{1}, \\
C^{(2)}= & -\left(N_{2}-\frac{h_{12}}{h_{11}} N_{1}\right) D \phi_{1} \wedge D \phi_{2}-D \phi_{2} \wedge C_{(12)} \\
& -D \phi_{1} \wedge A_{3}-\frac{1}{2} C_{1}+C_{(31)} \wedge A_{3}, \\
e^{2 \Phi}= & \frac{e^{2 \phi}}{h_{11}}, \quad C^{(0)}=N_{1} \\
C^{(4)}= & -\frac{1}{2} D \phi_{2} \wedge D \phi_{1} \wedge\left(C_{(2)}+2 C_{32} \wedge A_{3}-\frac{h_{12}}{h_{11}} C_{(1)}+2 C_{(31)} \wedge A_{3}\right) \\
& +\frac{1}{6}\left(C+3 C_{(3)} \wedge A_{3}\right) \wedge D \phi_{1}+d_{4}+\hat{d}_{3} \wedge D \phi_{1}, \\
D \phi_{1}= & d \phi_{1}-C_{(31)}, \quad D \alpha_{2}=d \phi_{2}+A_{2} .
\end{aligned}
$$

The forms $d_{4}, \hat{d}_{3}$ are determined by the self-duality conditions for the fiveform field strength. 
It will interesting to see how this procedure works for our example (5.31) associated to $S^{4}$. The relevant quantities that we need are

$$
\begin{aligned}
\phi_{D} & =\frac{3}{4} \log M_{\tau \tau}=-\frac{1}{4} \log \Delta, \\
N_{1} & =-\sin \widetilde{\rho} \sin \theta \cos \varphi, \quad N_{2}=-\sin \theta(\sin \varphi \cos \widetilde{\rho} \sin \widetilde{\theta}+\cos \varphi \cos \widetilde{\theta}), \\
\widetilde{h}_{11} & =\frac{1-(\sin \widetilde{\rho} \sin \theta \cos \varphi)^{2}}{\Delta^{1 / 2}}, \\
\widetilde{h}_{22} & =\frac{1-\sin ^{2} \theta(\cos \widetilde{\rho} \cos \widetilde{\theta} \sin \varphi+\cos \widetilde{\theta} \cos \varphi)^{2}}{\Delta^{1 / 2}}, \\
\widetilde{h}_{12} & =\frac{\left.\sin \widetilde{\rho} \cos \widetilde{\theta}+\sin \widetilde{\rho} \sin ^{2} \theta \cos \varphi \cos \widetilde{\rho} \cos \widetilde{\theta} \sin \varphi+\cos \widetilde{\theta} \cos \varphi\right)}{\Delta^{1 / 2}} .
\end{aligned}
$$

We have that $\widetilde{g}=\Delta^{1 / 6}\left(g_{A d S}+h\right) h$ being given in (5.25) and $\Delta$ given in (5.20). The resulting IIB background is in this case

$$
\begin{aligned}
g_{I I B} & =\frac{1}{\widetilde{h}_{11}}\left[\frac{1}{\sqrt{\Delta}}\left(D \phi_{1}\right)^{2}+\sqrt{\Delta}\left(D \phi_{2}\right)^{2}\right]+e^{2 \phi_{D} / 3} \widetilde{g}_{\mu \nu} d x^{\mu} d x^{\nu}, \\
B & =\frac{\widetilde{h}_{12}}{\widetilde{h}_{11}} D \phi_{1} \wedge D \phi_{2}+D \phi_{1} \wedge A_{1}, \quad e^{2 \Phi}=\frac{e^{2 \phi_{D}}}{\widetilde{h}_{11}}, \quad C^{0}=N_{1} \\
C^{2} & =-\left(N_{2}-\frac{\widetilde{h}_{12}}{\widetilde{h}_{11}} N_{1}\right) D \phi_{1} \wedge D \phi_{2}-D \phi_{1} \wedge A_{3}, \\
C^{4} & =\frac{1}{6} C^{3} \wedge D \phi_{1}+d^{4}+\widehat{d}^{3} \wedge D \phi_{2}, \quad D \phi_{1}=d \phi_{1} .
\end{aligned}
$$

The one-forms $A_{i}$ are defined in (5.23). The three-form $\widehat{d}^{3}$ and the fourform $d^{4}$ takes values in the eight-dimensional metric and are determined by imposing that the five-form field strength $F_{5}=d C_{4}$ is self-dual, i.e., $F_{5}=$ $*_{10} F_{5}$. In our case we have that

$$
\begin{aligned}
C^{3} & =-\frac{k \sin u}{3} \sinh ^{3} \rho d t \wedge d u \wedge d v \\
\frac{1}{6} d\left(C^{3} \wedge D \phi_{1}\right) & =-\frac{k}{6} \sin u \sinh ^{2} \rho d \rho \wedge d t \wedge d u \wedge d v \wedge d \phi_{1}
\end{aligned}
$$

and the self-duality condition is satisfied if $d^{4}$ is zero and

$$
C^{4}=\frac{1}{6}\left(C^{3} \wedge d \phi_{1}+\sqrt{\Delta} \widehat{C}^{3} \wedge D \phi_{2}\right)
$$

$\widehat{C}^{3}$ being such that $d \widehat{C}^{3}=\sqrt{\Delta} *_{8} d C^{3}$. The hodge star operation $*_{8}$ concerns the eight-dimensional metric $\widetilde{g}$. By comparing these expressions with (C:.1) 
we obtain that

$$
\begin{aligned}
F & =\widetilde{h}_{11}, \quad B_{12}=\frac{\widetilde{h}_{12}}{\widetilde{h}_{11}}, \quad B_{1}=B_{2}=0, \quad \widetilde{b}=\widetilde{c}=0, \\
C_{12} & =\frac{\widetilde{h}_{12}}{\widetilde{h}_{11}} N_{1}-N_{2}, \quad C_{1}=A_{3}, \quad C=N_{1}
\end{aligned}
$$

and it follows that all these quantities are defined by formulae (D:.3). From (C:.4) and (C:.5) we obtain directly the explicit deformed fields. The procedure is complete.

\section{References}

[1] G.T. Hooft, A planar diagram theory for strong interactions, Nucl. Phys. B 72 (1974), 461.

[2] E. Witten, Baryons in the $1 / N$ expansion; instantons, the Quark model, and the 1/N expansion, Nucl. Phys. B 160 (1979), 57; Nucl. Phys. B 149 (1979), 285.

[3] J. Maldacena, The large $N$ limit of superconformal field theories and supergravity, Adv. Theor. Math. Phys. 2 (1998), 231-252; Int. J. Theor. Phys. 38 (1999), 1113-1133.

[4] S. Gubser, I. Klebanov and A. Polyakov, Gauge theory correlators from noncritical string theory; Anti-De Sitter Space and holography; superconformal field theory on three-branes at a Calabi-Yau singularity, Phys. Lett. B 428 (1998), 105; E. Witten Adv. Theor. Math. Phys. 2 (1998), 253; I. Klebanov and E. Witten Nucl. Phys. B 536 (1998), 199.

[5] N. Itzhaki, J. Maldacena, J. Sonnenschein and S. Yankielowicz, Supergravity and the large $N$ limit of theories with sixteen supercharges, Phys. Rev. D 58 (1998), 046004.

[6] D. Berenstein, H. Nastase and J. Maldacena, Strings in flat space and Pp waves from $N=4$ Superyang-Mills, JHEP 0204 (2002), 013.

[7] S. Gubser, I. Klebanov and A. Polyakov, A semiclassical limit of the gauge/string correspondence, Nucl. Phys. B 636 (2002), 99.

[8] S. Frolov and A. Tseytlin, Semiclassical quantization of rotating superstring in $\operatorname{Ads}(5) \times S^{* *} 5$, JHEP 0206 (2002), 007.

[9] I. Klebanov and M. Strassler, Supergravity and a confining gauge theory: duality cascades and chi Sb resolution of naked singularities, JHEP 0008 (2000), 052. 
[10] G. Horowitz, The dark side of string theory: black holes and black strings, hep-th/9210119.

[11] N. Dorey, T. Hollowood and S. Kumar, S duality of the Leigh-Strassler Deformation via Matrix Models, JHEP 0212 (2002), 003.

[12] O. Lunin and J. Maldacena, Deforming field theories with $U(1) \times$ U(1) global symmetry and their gravity duals, JHEP 0505 (2005), 033.

[13] R. Leigh and M. Strassler, Exactly marginal operators and duality in four-dimensional $N=1$ supersymmetric gauge theory, Nucl. Phys. B 447 (1995), 95.

[14] M.T. Anderson, Geometric aspects of the AdS/CFT correspondence, hep-th/0403087.

[15] A. Chamseddine and M. Volkov, Non-Abelian BPS monopoles in $N=$ 4 gauged supergravity, Phys. Rev. Lett. 79 (1997) 3343; Non-Abelian solitons in $N=4$ gauged supergravity and leading order string theory, Phys. Rev. D57 (1998) 6242; J. Maldacena and C. Nunez Towards the large $N$ limit of pure $N=1$ super Yang Mills, Phys. Rev. Lett. 86 (2001) 588 .

[16] E. Witten, Branes and the dynamics of QCD, Nucl. Phys. B 507 (1997), 658.

[17] H. Liu, K. Rajagopal and U. Wiedemann, Calculating the jet quenching parameter from Ads/Cft; Jet quenching at finite t' Hooft Coupling and chemical potential from Ads/Cft, Phys. Rev. Lett. 97 (2006), 182301; N. Armesto, J. Edelstein and J. Mas, JHEP 0609 (2006), 039.

[18] S. Gubser, Einstein manifolds and conformal field theories, Phys. Rev. D 59 (1999), 025006.

[19] P. Bozhilov, Exact rotating membrane solutions on A G(2) manifold and their semiclassical limits; Rotating strings and D2-Branes in type iia reduction of $M$-Theory on $G(2)$ manifold and their semiclassical limits, JHEP 0603 (2006), 001; JHEP 0608 (2006), 029.

[20] S. Hartnoll and C. Nunez, Rotating membranes on G(2) manifolds, logarithmic anomalous dimensions and $N=1$ duality, JHEP 0302 (2003), 049.

[21] U. Gursoy and C. Nunez, Dipole deformations of $N=1$ sym and supergravity backgrounds with $U(1) \times U(1)$ global symmetry, Nucl. Phys. B 725 (2005), 45.

[22] S. Salamon, Quaternionic Kähler manifolds, Invent. Math. 67 (1982), 143; L. Berard Bergery, New examples of Einstein metrics, Mathematische Arbeitstagung Bonn, 1979. 
[23] R. Bryant, Metrics with exceptional holonomy; On the construction of some complete metrics with exceptional holonomy, Ann. Math. 126 (1987), 525; R. Bryant and S. Salamon, Duke Math. J. 58 (1989), 829.

[24] G. Gibbons, D. Page and C. Pope, Einstein Metrics on $S^{* * 3} R^{* * 3}$ and $R^{* *} 4$ bundles, Commun. Math. Phys. 127 (1990), 529.

[25] M. Berger, Sur les groupes d'holonomie des varietes a connexion affine et des varietes riemanniennes, Bull. Soc. Math. France 83 (1955), 279.

[26] A. Wolf, Complex homogeneous contact manifolds and quaternionic symmetric spaces; Compact quaternionic spaces, J. Math. Mech. 14 (1965), 1033; D. Alekseevskii, Func. Anal. Appl. 2 (1968), 11.

[27] E. Bergshoeff, S. Cucu, T. de Wit, J. Gheerardyn, S. Vandoren and A. Van Proeyen, The map between conformal hypercomplex/hyperKahler and quaternionic(-Kahler) geometry, Commun. Math. Phys. 262 (2006), 411.

[28] J. Ishihara, Quaternion Kahlerian manifolds, J. Diff. Geom. 9 (1974), 483.

[29] N. Hitchin, Stable forms and special metrics, math.dg/0107101.

[30] A. Bilal, J. Derendinger and K. Sfetsos, (Weak) G(2) holonomy from selfduality, flux and supersymmetry, Nucl. Phys. B 628 (2002), 112.

[31] A. Bilal and S. Metzger, Compact weak G(2) manifolds with conical singularities, Nucl. Phys. B 663 (2003), 343.

[32] R. D'Auria Castellani, and P. Fre, $S U(3) \times S U(2) \times U(1)$ from $D=$ 11 supergravity, Nucl. Phys. B 239 (1984), 610.

[33] R. D Auria, P. Fre and P. van Nieuwenhuisen, $N=2$ Matter coupled supergravity from compactification on a coset $G / H$ possessing an additional killing vector, Phys. Lett. B 136 (1984), 347.

[34] D. Page and C. Pope, Einstein Metrics on quaternionic line bundles, Phys. Lett. B 147 (1984), 55.

[35] H. Pedersen and Y.S. Poon, Hyper-Kähler metrics and a generalization of the Bogomolny equations, Comm. Math. Phys. 117 (1988), 569.

[36] D. Page, Inhomogeneous Einstein Metrics on complex line bundles; Einstein Metrics on quaternionic line bundles, Class. Quant. Grav. 4 (1987), 213; D. Page and C. Pope, Class. Quant. Grav. 3 (1986), 249.

[37] H. Lu, C.N. Pope and J. Vazquez-Poritz, A new construction of Einstein-Sasaki metrics In $D>=7$, hep-th/0512306.

[38] M. Cvetic, H. Lu, Don N. Page and C.N. Pope, New Einstein-Sasaki spaces in five and higher dimensions; New Einstein-Sasaki and Einstein spaces from Kerr-de Sitter, Phys. Rev. Lett. 95 (2005), 071101; hep-th/0505223; H. Lu, D. Page and C. Pope, New inhomogeneous 
Einstein metrics on sphere bundles over Einstein-Kahler manifolds Phys. Lett. B 593 (2004), 218.

[39] A. Bergman and C. Herzog, The volume of some nonspherical horizons and the Ads/Cft correspondence, JHEP 0201 (2002), 030.

[40] V. Apostolov, D. Calderbank, P. Gauduchon and C. TonnesenFriedman, Hamiltonian 2-forms in Kahler geometry, IV Weakly Bochner-flat Kahler manifolds, arXiv:math/0511119; Hamiltonian 2-forms in Kahler geometry, II Global Classification, J. Diff. Geom. 68 (2004) 277-345; Hamiltonian 2-forms in Kahler geometry, I General Theory, J. Diff. Geom. 73 (2006) 359-412.

[41] D. Martelli and J. Sparks, Toric Sasaki-Einstein Metrics on $S^{* *} 2 \times$ $S^{* *}$ 3, Phys. Lett. B 621 (2005), 208.

[42] W. Chen, H. Lu, C.N. Pope and J. Vazquez-Poritz, A note on Einstein Sasaki Metrics in D> 7, Class. Quant. Grav. 22 (2005), 3421.

[43] J. Gauntlett, D. Martelli, J. Sparks and S.T.Yau, Obstructions to the existence of Sasaki-Einstein Metrics, hep-th/0607080.

[44] J. Gauntlett, D. Martelli, J. Sparks and Daniel Waldram, A new infinite class of Sasaki-Einstein manifolds, Adv. Theor. Math. Phys. 8 (2004), 987.

[45] D. Martelli and J. Sparks, Toric geometry, Sasaki-Einstein manifolds and a new infinite class of AdS/CFT duals, Commun. Math. Phys. 262 (2006), 51.

[46] D. Fabbri, P. Fre, L. Gualtieri, C. Reina, A. Tomasiello, A. Zaffaroni and A. Zampa, 3D superconformal theories from Sasakian sevenmanifolds: new nontrivial evidences for AdS_4/CFT_3, Nucl. Phys. B 577 (2000), 547.

[47] M. Billo, D. Fabbri, P. Fre, P. Merlatti and A. Zaffaroni, Rings of short $N=3$ superfields in three dimensions and $M$-theory on $A d S(4) \times N(0,1,0)$, Class. Quant. Grav. 18 (2001), 1269.

[48] S. Benvenuti, S. Franco, A. Hanany, D. Martelli and J. Sparks, An infinite family of superconformal quiver gauge theories with SasakiEinstein Duals, JHEP 0506 (2005), 064.

[49] S. Benvenuti, A. Hanany and P. Kazakopoulos, The toric phases of the $Y^{* *} P, Q$ Quivers; A new infinite class of quiver gauge theories, JHEP 0507 (2005), 021; A. Hanany, P. Kazakopoulos and B. Wetch, JHEP 0508 (2005), 054.

[50] D. Martelli, J. Sparks and S.T. Yau, Sasaki-Einstein manifolds and volume minimisation; semiclassical strings in Sasaki-Einstein manifolds and long operators in $N=1$ gauge theories, Commun. Math. Phys. 268 (2006), 39; hep-th/0603021. 
[51] S. Benvenutti and M. Kruczenski, From Sasaki-Einstein spaces to quivers via Bps Geodesics: $L^{* *} P, Q \mid R$; Supersymmetric solutions based on $Y^{* *} P, Q$ and $L^{* *} P, Q, R$, JHEP 0610 (2006), 051; JHEP 0604 (2006), 033; K. Sfetsos and D. Zoakos, Phys. Lett. B 625 (2005), 135.

[52] M. Cvetic, G.W. Gibbons, H. Lu and C.N. Pope, Hyper-Kahler Calabi Metrics, $L^{2}$ harmonic forms, resolved M2-Branes, and AdS_4/CFT_3 correspondence, Nucl. Phys. B 617 (2001), 151.

[53] I. Klebanov and A. Murugan, Gauge/gravity duality and warped resolved conifold, hep-th/0701064.

[54] W. Chen, M. Cvetic, H. Lu, C. Pope and J. Vazquez-Poritz, Resolved Calabi-Yau cones and flows from $L^{* *}(A b c)$ superconformal field theories, hep-th/0701082.

[55] D. Gepner and S. Pal, Branes in $L(P, Q, R)$, Phys. Lett. B 622 (2005), 136.

[56] C. Ahn, Penrose limit of $A d s(4) \times V(5,2)$ and operators with large $R$ charge; More on Penrose limit of $\operatorname{Ads}(4) \times Q^{* *}(1,1,1)$, Mod. Phys. Lett. A 17 (2002), 2067; Phys. Lett. B 539 (2002), 281.

[57] C. Ahn Comments on Penrose limit of $\operatorname{AdS}(4) \times M(1,1,1)$; More Penrose limit of $A d S(4) \times N(0,1,0)$ and $N=3$ gauge theory, Mod. Phys. Lett. A 17 (2002), 1847; Phys. Lett. B 540 (2002), 111.

[58] A. Butti, D. Forcella and A. Zaffaroni, The Dual superconformal theory for $L^{* *}$ Pqr manifolds; Counting Bps Baryonic operators in Cfts with Sasaki-Einstein duals, JHEP 0509 (2005), 018; hep-th/0611229.

[59] F. Canoura, J. Edelstein and A. Ramallo, D-Brane probes on $L(A, B, C)$ superconformal field theories; Canoura: Supersymmetric branes on $\operatorname{Ads}(5) \times Y^{* *} P, Q$ and their field theory duals, JHEP 0609 (2006), 038; F. Canoura; J. Edelstein, L. Pando-Zayas, A. Ramallo and D. Vaman, JHEP 0603 (2006), 101.

[60] M. Cvetic, H. Lu, Don N. Page and C.N. Pope New Einstein-Sasaki spaces in five and higher dimensions, Phys. Rev. Lett. 95 (2005), 071101.

[61] K. Lee and H. Yee, New $A d S(4) \times X(7)$ geometries with $C N=6$ in $M$ theory, hep-th/0605214; H. Yee, AdS/CFT with tri-Sasakian manifolds, hep-th/0612002.

[62] J. Gauntlett, S. Lee, T. Mateos and D. Waldram, Marginal deformations of field theories with Ads(4) duals, JHEP 0508 (2005), 030 .

[63] C. Ahn and J. Vazquez-Poritz, Deformations of flows from type IIB supergravity, Class. Quant. Grav. 23 (2006), 3619. 
[64] C. Ahn and J. Vazquez-Poritz, Marginal deformations with $U(1)^{* *} 3$ global symmetry, JHEP 0507 (2005), 032.

[65] C. Ahn and J. Vazquez-Poritz, From marginal deformations to confinement, JHEP 0606(2006), 061.

[66] H. Pedersen and Y.S. Poon, Hamiltonian constructions of KählerEinstein metrics and Kähler metrics of constant scalar curvature, Comm. Math. Phys. 136 (1991), 309.

[67] O. Santillan, A Kahler-Einstein inspired anzatz for Spin(7) holonomy metrics and its solution, hep-th/0609088.

[68] R. Bielawski, Ricci flat Kaehler metrics on canonical bundles, math.DG/0006144.

[69] A. Hwang and M. Singer, A momentum construction for circleinvariant Kahler metrics, math.DG/9811024.

[70] A. Besse, Einstein manifolds, Springer, New York, 1987.

[71] R. Ward, Minitwistor spaces and Einstein-Weyl spaces, Class. Quant. Grav. 7 (1990), L95.

[72] V. Apostolov, T. Draghici and A. Moroianu, A splitting theorem for Kahler manifolds whose Ricci tensors have constant eigenvalues, math.DG/0007122.

[73] V. Apostolov and P. Gauduchon, Self-dual Einstein Hermitian four manifolds, math.DG/0003162.

[74] R. Bryant, Bochner-Kähler metrics, J. Amer. Math. Soc. 14 (2001), 623.

[75] C. Boyer and C. Galicki, Sasakian geometry, hypersurface singularities, and Einstein metrics, math.dg/0405256.

[76] G. Mandal, N. Suryanarayana and S. Wadia, Aspects of semiclassical strings in Ads(5), Phys. Lett. B 543 (2002), 81.

[77] K. Galicki, A generalization of the momentum mapping construction for quaternionic Kähler manifolds; Quaternionic reduction and quaternionic orbifolds; Hyper-Kähler metrics and supersymmetry, Comm. Math. Phys. 108 (1987), 117; K. Galicki and H. Lawson, Math. Ann. 288 (1988), 1; N.J. Hitchin, A. Karlhede, U. Lindstrom and M. Rocek, Comm. Math. Phys. 108 (1987), 535.

[78] P.B. Kronheimer, The construction of ALE spaces as hyper-Kähler quotients; A Torelli type theorem for gravitational instantons, J. Diff. Geom. 29 (1989), 665; P.B. Kronheimer, J. Diff. Geom. 29 (1989), 685.

[79] A. Swann, HyperKähler and quaternionic Kähler geometry, Math. Ann. 289 (1991), 421. 
[80] L. Anguelova, M. Rocek and S. Vandoren, HyperKahler cones and orthogonal Wolf spaces, JHEP 0205 (2002), 064.

[81] J. Plebanski and M. Demianski, Rotating, charged, and uniformly accelerating mass in general relativity, Ann. Phys. 98 (1975), 98.

[82] K. Zoubos, Holography and quaternionic Taub NUT, JHEP 0212 (2002), 037.

[83] A. Chamblin, R. Emparan, C. Johnson and R. Myers, Large N phases, gravitational instantons and the nuts and bolts of AdS holography; Surface terms as counterterms in the AdS/CFT correspondence, Phys. Rev. D 59 (1999), 064010; R. Emparan, C. Johnson and R. Myers, Phys. Rev. D 60 (1999), 104001.

[84] S. Hawking, C. Hunter and D. Page, Nut charge, anti-de Sitter space and entropy, Phys. Rev. D 59 (1999), 044033.

[85] K. Behrdnt, G. Dall'Agata, D. Lust and S. Mahapatra, Intersecting Six-branes from new seven manifolds with G(2) holonomy; Singular seven manifolds with G(2) holonomy and intersecting six-branes, JHEP 0208 (2002), 027; K. Behrdnt, Nucl. Phys. B 635 (2002), 158.

[86] E. Ivanov and G. Valent, Quaternionic Taub - NUT from the harmonic space approach; Harmonic space construction of the quaternionic Taub-NUT metric; Quaternionic extension of the double Taub - NUT metric, Phys. Lett. B 445 (1998), 60; Class. Quant. Grav. 16 (1999), 1039; P. Casteill, E. Ivanov and G. Valent Phys. Lett. B 508 (2001), 354 .

[87] A. Galperin, E. Ivanov, O. Ogievetsky, Harmonic space and quaternionic manifolds, Ann. Phys. 230 (1994), 201-249.

[88] O. Santillan, A Construction of G(2) holonomy spaces with torus symmetry, Nucl. Phys. B 660 (2003), 169.

[89] L. Anguelova and C. Lazaroiu, M Theory compactifications on certain 'Toric' cones of G(2) holonomy, JHEP 0301 (2003), 066.

[90] L. Anguelova and C. Lazaroiu, $M$ Theory on 'Toric' G(2) cones and its type ii reduction; Domain walls of $N=2$ supergravity in fivedimensions from hypermultiplet moduli spaces, JHEP 0210 (2002), 038; L. Anguelova and C. Lazaroiu, JHEP 0209 (2002), 053.

[91] U. Gursoy, C. Nunez and M. Schvellinger, Rg flows from $\operatorname{spin}(7), C y$ 4 fold and Hk manifolds to Ads, Penrose limits and Pp waves, JHEP 0206 (2002), 015.

[92] A. Ashtekar, T. Jacobson and L. Smolin, A new characterization of half flat solutions to Einstein's equation, Comm. Math. Phys. 115 (1988), 631. 
[93] D. Calderbank and H. Pedersen, Selfdual Einstein metrics with torus symmetry, J. Diff. Geom. 60 (2002), 485.

[94] D. Calderbank and M. Singer, Continued fractions and Einstein manifolds of infinite topological type, hep-th/0508614; Toric selfdual Einstein metrics on compact orbifolds, Duke. Math. J 133 (2006), 237; Einstein metrics and complex singularities, Invent. Math. 156 (2004), 405.

[95] D. Calderbank and H. Pedersen, Selfdual spaces with complex structures, Einstein-Weyl geometry and geodesics, Ann. Inst. Fourier 50 (2000), 921.

[96] D. Calderbank and P. Tod, Einstein metrics, hypercomplex structures and the Toda field equation, Differ. Geom. Appl. 14 (2001), 199.

[97] D. Calderbank, The geometry of the Toda equation, J. Geom. Phys. 36 (2000), 152.

[98] R. Bielawski and A. Dancer, The geometry and topology of toric hyperKahler manifolds, Comm. Anal. Geom. 8 (2000), 727.

[99] H. Pedersen, Spinning top motions and monopoles, Math. Ann. 274 (1986), 35.

[100] P. Casteill, E. Ivanov, G. Valent, Quaternionic extension of the double taub - nut metric; $U(1) \times U(1)$ quaternionic metrics from harmonic superspace, Phys. Lett. B 508 (2001), 354-364; Nucl. Phys. B 627 (2002), 403.

[101] T. Buscher, A Symmetry of the String Background Field Equations, Phys. Lett. B 194 (1987), 59.

[102] D. Joyce, Explicit construction of self-dual four-manifolds, Duke. Math. J. 77 (1995), 519.

[103] S.V. Ketov, Summing up D instantons in $N=2$ Phys. B 649 (2003), 365.

[104] S.V. Ketov, Instanton induced scalar potential for the universal hypermultiplet; D instantons and matter hypermultiplets, Nucl. Phys. B 656 (2003), 63; S.V. Ketov Phys. Lett. B 558 (2003), 119.

[105] I. Antoniadis, R. Minasian, S. Theisen and P. Vanhove, String loop corrections to the universal hypermultiplet, Class. Quant. Grav. 20 (2003), 5079.

[106] T. Eguchi and A. Hanson, Asymptotically flat selfdual solutions to Euclidean gravity; Selfdual solutions to Euclidean gravity, Phys. Lett. B 74 (1978), 249; Ann. Phys. 120 (1979), 82.

[107] A. Gray, Weak holonomy groups, Math. Z. 123 (1971), 290. 
[108] E. Cremmer, B. Julia, H. Lu and C.N. Pope, Dualization of dualities, Nucl. Phys. B 523 (1998), 73.

[109] B. Wit, M. Rocek and S. Vandoren, Hypermultiplets, HyperKahler cones and quaternion Kahler geometry; Gauging isometries on HyperKahler cones and quaternion Kahler manifolds, JHEP 0102 (2001), 039; Phys. Lett. B 511 (2001), 302.

[110] D. Robles-Llana, F. Saueressig and S. Vandoren, String loop corrected hypermultiplet moduli spaces, JHEP 0603 (2006), 081.

[111] O. Santillan and A. Zorin, Toric HyperKahler manifolds with quaternionic Kahler bases and supergravity solutions, Commun. Math. Phys. 255 (2005), 33.

[112] L. Anguelova, M. Rocek and S. Vandoren, HyperKahler cones and orthogonal Wolf spaces; Quaternion-Kahler Spaces, HyperKahler cones, and the C-Map, JHEP 0205 (2002), 064; M. Rocek, C. Vafa and S. Vandoren math.DG/0603048.

[113] G. Gibbons and S. Hawkings, Gravitational multi-instantons, Phys. Lett. B 78 (1978), 430.

[114] T. Eguchi and A. Hanson, Asymptotically flat selfdual solutions to Euclidean gravity; Eguchi: Selfdual solutions to Euclidean gravity; gravitational instantons, Phys. Lett. B 74 (1978), 249; Ann. Phys. 120 (1979), 82; Gen. Rel. Grav. 11 (1979), 315.

[115] S. Hawking, Gravitational instantons, Phys. Lett. A 60 (1977), 81. 